\title{
Sensing and Filtering: A Fresh Perspective Based on Preimages and Information Spaces
}

\author{
Steven M. LaValle \\ Department of Computer Science \\ University of Illinois
}

\begin{abstract}
This paper presents an unusual perspective on sensing uncertainty and filtering with the intention of understanding what information is minimally needed to achieve a specified task. Information itself is modeled using information space concepts, which originated from dynamic game theory (rather than information theory, which was developed mainly for communication). The guiding principle in this paper is avoid sensing, representing, and encoding more than is necessary. The concepts and tools are motivated by many tasks of current interest, such as tracking, monitoring, navigation, pursuit-evasion, exploration, and mapping. First, an overview of sensors that appear in numerous systems is presented. Following this, the notion of a virtual sensor is explained, which provides a mathematical way to model numerous sensors while abstracting away their particular physical implementation. Dozens of useful models are given, each as a mapping from the physical world to the set of possible sensor outputs. Preimages with respect to this mapping represent a fundamental source of uncertainty: These are equivalence classes of physical states that would produce the same sensor output. Pursuing this idea further, the powerful notion of a sensor lattice is introduced, in which all possible virtual sensors can be rigorously compared. The next part introduces filters that aggregate information from multiple sensor readings. The integration of information over space and time is considered. In the spatial setting, classical triangulation methods are expressed in terms of preimages. In the temporal setting, an information-space framework is introduced that encompasses familiar Kalman and Bayesian filters, but also introduces a novel family called combinatorial filters. Finally, the planning problem is presented in terms of filters and information spaces. The paper concludes with some discussion about connections to many related research fields and numerous open problems and future research directions.
\end{abstract}




\section{Introduction}

Think about the devices we build that intermingle sensors, actuators, and computers. Whether they be robot systems, autonomous vehicles, sensor networks, or embedded systems, they are completely blind to the world until we equip them with sensors. All of their accomplishments rest on their ability to sift through sensor data and make appropriate decisions. This article therefore takes a completely sensor-centric view for designing these systems.

It is tempting (and common) to introduce the most complete and accurate sensors possible to eliminate uncertainties and learn a detailed, complex model of the surrounding world. In contrast, this article heads in the opposite direction by starting with sensing first and then understanding what information is minimally needed to solve specific tasks. If we can accomplish our mission without knowing certain details about the world, then the overall system may be more simple and robust.

This can be partly understood by considering computational constraints. One way or another, we want computers to process and interpret the data obtained from sensors. The computers might range from limited embedded systems to the most powerful computer systems. The source of their data is quite different from classical uses of computers, in which data are constructed by humans, possibly with the help of software. When data are obtained from sensors, there is a direct sensor mapping from the physical world onto a set of sensor readings. Even though sensors have been connected to computers for decades, there has been a tendency to immediately digitize the sensor data and treat it like any other data. With the proliferation of cheap sensors these days, it is tempting to easily gather hordes of sensor data and google them for the right answer. This may be difficult to accomplish, however, without carefully understanding the sensor mapping. A large part of this article is therefore devoted to providing numerous definitions and examples of practical sensor mappings.

When studying sensors, one of the first things to notice is that most sensors leave a huge amount of ambiguity with regard to the state of the physical world. Example: How much can we infer about the world when someone triggers an infrared sensor to turn on a bathroom sink? In many fields, there is a common temptation to place enough powerful sensors so that as much as possible about the physical world can be reconstructed. The idea is to give a crisp, complete model that tends to make computers happy. In this article, however, we argue that it is important to start with the particular task and then determine its information requirements: What critical pieces of information about the world do we need to maintain, while leaving everything else ambiguous? The idea is to "handle" uncertainty by avoiding big models whenever possible. This is hard to accomplish if we design a general purpose robot with no clear intention in mind; however, most devices appearing in practice have specific, well-defined tasks to perform.

Depending on your background, there might be surprises in this article:

1. Discrete vs. continuous: Not very important: Even though computation is discrete and the physical world is usually modeled with continuous spaces, the distinction is not too important here. The field of hybrid systems is devoted to the interplay between continuous models, usually expressed with differential equations, and discrete computation models. The point in this article, however, is to study sensor mappings. These may be from continuous to continuous spaces, continuous to discrete, or even discrete to discrete (if the physical world is modeled discretely).

2. Information spaces, not information theory: As an elegant and useful mathematical framework for characterizing information transmitted through a noisy channel, Shannon's information theory is extremely powerful. The concepts are fundamental to many fields; 


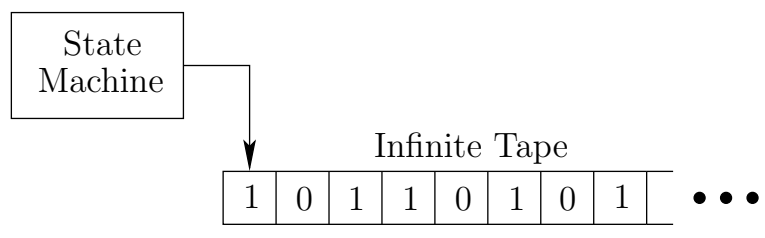

(a)

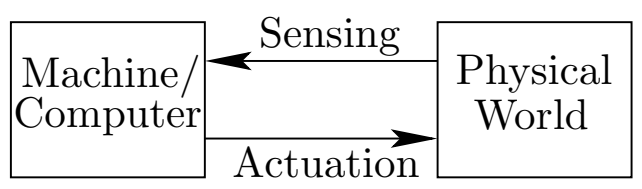

(b)

Figure 1: (a) For classical computation, the full state is given by the finite machine state, the head position, and the binary string written on the tape. (b) In this paper, there is both an internal computational state and an external physical state.

however, information spaces were formulated since the 1940s in the context of game theory and control theory for systems that are unable to determine their state. Thus, this article talks more about how to accomplish tasks in spite of huge amounts of ambiguity in state, rather than measuring information content, using entropy-based constructs. There may indeed be interesting connections between the two subjects, but they are not well understood and are therefore not covered here.

3. Perfectly accurate and reliable sensors yield huge amounts of uncertainty: Uncertainty in sensing systems is usually handled by formulating statistical models of disturbance. For example, a global positioning system (GPS) may output location coordinates, but a Guassian noise model might be used to account for the true position. It is important, however, to study the often neglected source of uncertainty due simply to the sensor mapping. Consider the sensor pad at the entrance to a parking garage or drive-through restaurant. It provides one bit of information, usually quite reliably and accurately. It performs its task well, in spite of enormous uncertainty about the world: What kind of car drove over it? Where precisely did the car drive? How fast was it going? We are comfortable allowing this uncertainty to remain uncertain. We want to study these situations broadly. This is complementary to the topic of noisy sensors, and both issues can and should be addressed simultaneously. This article, however, focuses mainly on the underrepresented topic of uncertainty that arises from the sensor mapping.

Based on the discussion above, it is clear that sensing and computation are closely intertwined. For robotic devices, actuation additionally comes into play. This means that commands are issued by the computer, causing the device to move in the physical world. Therefore, many problems of interest mix all three: sensing, actuation, and computation. Alternative names for sensing are perception or even learning, but each carries distinct connotations. A broader name for actuation is control, which may or not refer to forcing changes in the physical world. Based on this three-way mixture and its increasing relevance, we are forced more than ever to develop new mathematical abstractions and models that reduce complexity and meet performance goals.

Figure 1 shows a conceptual distinction between classical computation and the three-way mixture considered in this paper. In Figure 1(a), the Turing machine model is shown, in which a state machine interacts with a boundless binary tape. This and other computation models represent useful, powerful abstractions for ignoring the physical world. Figure 1(b) emphasizes the interaction between the physical world and a computer. Imagine discarding the Turing tape and interacting directly with a wild, unknown, chaotic world through sensing and actuation.

A natural question arises: What is the "state" of this system? In the case of the Turing machine the full state is given by: the finite machine state, head position, and the binary string written 
on the tape. For Figure 1(b), this becomes replaced by two kinds of states: internal and external. The internal state corresponds to the state inside of the computation box. Some or all of the internal state will be called an information state (or I-state), to be defined later. The external state corresponds to the state of the physical world. The internal state is closer to the use of state in computer science, whereas the external state is closer to its use in control theory. The internal vs. external distinction is more important than discrete vs. continuous; either kind of state may be continuous or discrete.

These internal states will be defined to live in an information space (or I-space), which is where filtering and planning problems naturally live when sensing is involved. In this article, we will define and interpret these spaces in many settings. A continuing mission is to make these spaces as small as possible while being able to efficiently compute over them and to understand their connection to the external states.

Here are some key themes to take from this article:

- Start from the task and try to understand what information is actually required to be extracted from the physical world.

- Since sensors leave substantial uncertainty about the physical world, they are best understood as inducing partitions of the external state space into indistinguishable classes of physical states.

- We can design combinatorial filters that are structurally similar to Bayesian or Kalman filters, but involve no probabilistic models. These are often dramatically simpler in complexity. They are also perfectly compatible with probabilistic reasoning: Stochastic models can be introduced over them.

- There is no problem defining enormous physical state spaces, provided that we do not directly compute over them. However, state estimation or recovery of a particular state in a giant state space should be avoided if possible.

- Virtual sensor models provide a powerful intermediate abstraction that can be implemented by many alternative physical sensing systems.

The remainder of this paper is divided into four main parts:

1. Physical sensors: Before going into mathematical models, a broad overview of real sensors will be given along with discussions about what we would like to sense.

2. Virtual sensors: This part introduces mathematical models of sensors that are abstracted away from the particular physical implementation. Using a definition of the physical state space, a sensor is defined as a mapping from physical states to data that can be measured.

3. Filtering: Information accumulates from multiple sensor readings over time or space and needs to be efficiently combined. Spatial filters generalize ancient triangulation methods and combine information over space. For temporal filters, we find and attempt to "live" in the smallest I-space possible, given the task. The concepts provide a generalization of Kalman and Bayesian filters. The new family includes reduced-complexity filters, called combinatorial filters, that avoid physical state estimation.

4. Discussion: In the final part, the transition to planning is briefly considered. A plan specifies actuation primitives (or actions) that are conditioned on the I-states maintained in a filter and manipulate the world to achieve tasks. Related research and future research challenges are then presented to end the paper. 


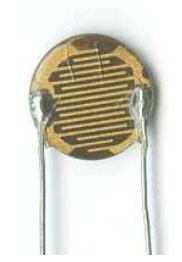

(a) Light-dependent resistor

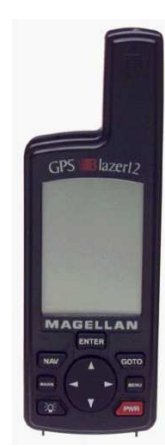

(b) GPS unit

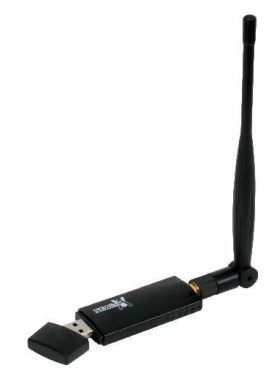

(c) Wireless card

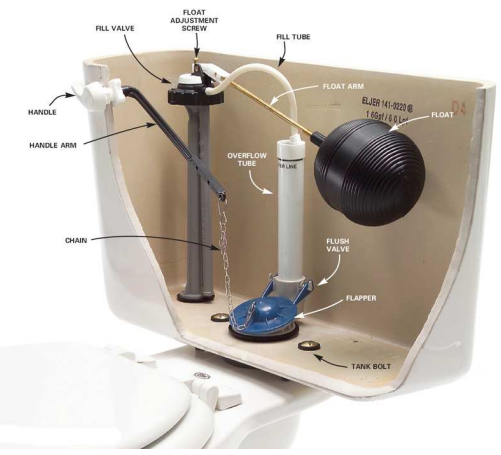

(d) Toilet float mechanism

Figure 2: Some examples of sensors.

Filtering and planning can be distinguished by being passive and active, respectively. A filtering problem might require making inferences, such as counting the number of people in a building or determining the intent of a set of autonomous vehicles. A planning problem usually disturbs the environment, for example by causing a robot to move a box across the floor.

\section{Physical Sensors}

\subsection{What Is a Sensor?}

What is a sensor? Even though we are quick to find examples, it is a difficult question to answer precisely. Consider some devices shown in Figure 2. To consider each a sensor, it seems that the device must be used by a larger system for some form of inference or decision making. The lightdependent resistor (LDR) in Figure 2(a) alters the current or voltage when placed in a circuit. It can be considered as a transducer, which is a device that converts one form of energy into another; the LDR converts light into an electrical signal. When connected to a larger system, such as a robot, we will happily consider it as a sensor. Figure 2(b) shows a complete global position system (GPS) device, which measures position, orientation, and velocity information. As a black box, it produces information similar to the LDR placed into a tiny circuit; however, its operation is much complex because it measures phase shifts between signals emitted by orbiting satellites. When connected to a larger system, its precision and error characteristics are much harder to analyze (for example, are trees blocking satellites?). The process occurring inside the sensor is much more complex than for a simple transducer. A sensor could quite easily be more complex than a robot that uses it.

We might take a device that was designed for another purpose and abuse it into being a sensor. For example, the wireless card in Figure 2(c) was designed mainly for communications; however, it can also be configured in a larger system to simply serve as a signal meter. It was illustrated in [33] that when used as a sensor, it provides powerful localization information. This should cause us to look around and abuse any device we can find into performing as a sensor.

Finally, it seems that the float mechanism in a toilet water tank, shown in Figure 2(d), serves as a sensor to determine when to shut off the flow valve. This is perfectly fine as a sensor in a purely mechanical system, but in this paper, we consider only sensors that provide input to computer systems.

Based on these examples, it seems best to avoid having a precise definition of a sensor. We will 


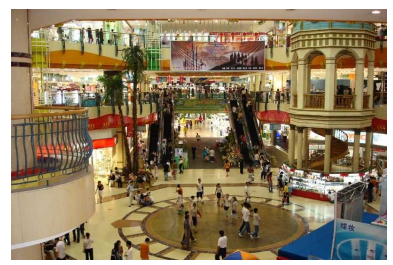

(a) Shopping mall

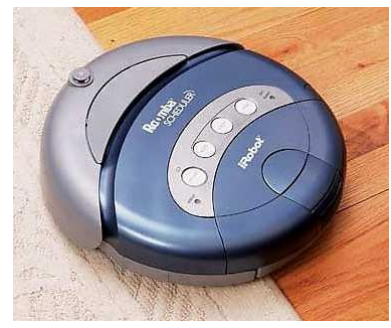

(e) Roomba

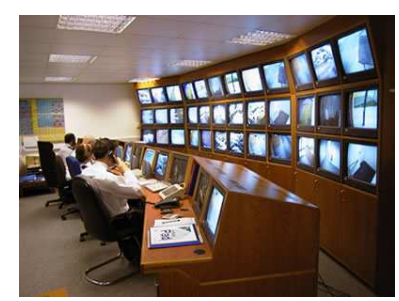

(b) Control room

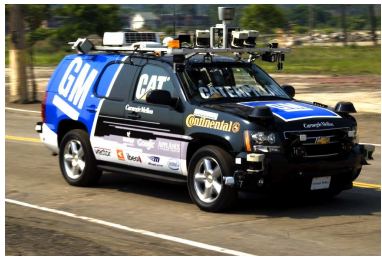

(f) CMU Boss

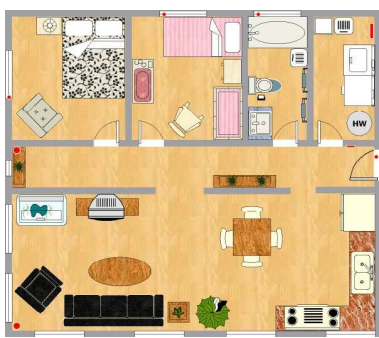

(c) Assisted living

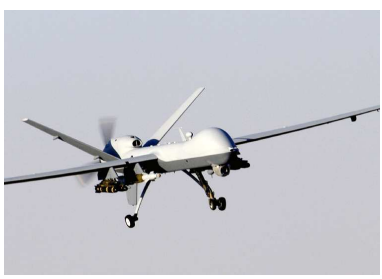

(g) UAV

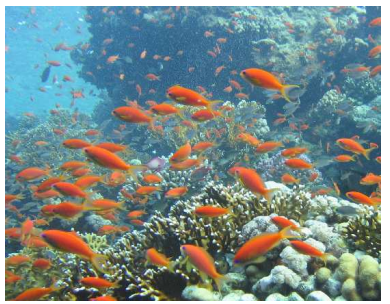

(d) Coral reef

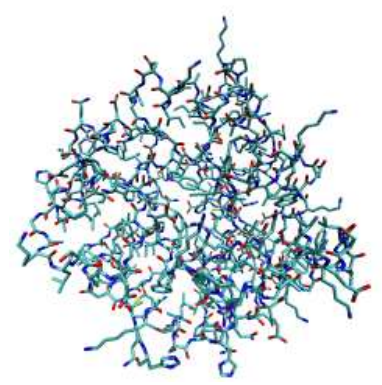

(h) Protein

Figure 3: Several motivational settings in which we would like to use sensors to monitor or control the environment.

talk about numerous sensors, with the understanding they are just devices that respond to external stimuli and provide signals to a larger system. The next step is to consider the kinds of scenarios in which we will be placing sensors.

\subsection{Where Might We Want to Use Sensors?}

It is difficult to exhaustively list settings where sensors might be placed. To nevertheless provide some perspective on the kinds places where the concepts from this paper may apply, consider the motivating examples shown in Figure 3. Figure 3(a) shows a shopping mall with numerous people moving around. Common tasks could be monitoring activities for security or studying consumer habits (see VideoMining solutions). Related to this, Figure 3(b) shows a security control room in which video is monitored from numerous sources within the same building. How much can be reconstructed about the movements of people, as they become visible to various cameras? We might want to count people, estimate their flow, or classify them. Now consider a home setting, in which security is a common problem; see Figure 3(c). An increasingly important engineering problem is to monitor activities of people who require assisted living. By keeping track of their movements, changes in their behavior can be detected. Furthermore, if they become trapped, an alarm can be sounded for emergency action. In this setting, people prefer not to be monitored by cameras for privacy reasons. What kind of minimally invasive sensors can be used to accomplish basic monitoring tasks? Figure 3(d) shows a similar task, but instead involves monitoring wildlife. Imagine gathering data on air, land, or sea animals for scientific and conservation purposes.

The examples so far have involved passive monitoring, without directly interfering with the environment. Figures 3(e)-(g) show three examples of robotic vehicles that interact with their environment. Sensing is combined with actuation to move vehicles. In the 3(e), a low-cost robot vacuums floors inside of homes. 
Figure 3(f) shows the vehicle that won the DARPA Urban Challenge, which involved driving autonomously through a town while taking into account traffic rules and other vehicles. Automated driving is gaining increasing interest for both transportation and military use. We can imagine robots or autonomous vehicles in the sea, on land, in the air, and in space; Figure 3(g) shows an autonomous aerial vehicle (UAV). Other robotic examples include arms that weld in a factory (as in PUMA or ABB robots), mobile robots that arrange inventory in a warehouse (as in Kiva Systems), and humanoids.

Finally, some of the concepts from this paper may apply well beyond the scope of the examples here. For example, the problem of measuring protein structure, shown in Figure 3(h), can be viewed as trying to reconstruct as much information possible from limited measurements (which are obtained by sensors, such as mass spectroscopy and NMR).

\subsection{What Physical Quantities Are Sensable?}

Based on the numerous examples from Section 2.2, it is helpful to group together similar phenomena that can be measured from the surrounding physical world. Consider the following categories of physical quantities:

Spatial: displacement, velocity, acceleration, distance to something, proximity, position, attitude, area, volume, level/tilt, motion detection

Temporal: clock, chronometer (elapsed time), frequency.

Electromagnetic: voltage, current, power, charge, capacitance, inductance, magnetic field, light intensity, color. These may operate within a circuit or within open space.

Mechanical: solid (mass, weight, density, force, strain, torque), fluid (acoustic, pressure, flow, viscosity), thermal (temperature), calories.

Other: chemical (composition, pH, humidity, pollution, ozone), radiation (nuclear), biomedical (blood flow, pressure).

Clearly a wide variety of phenomena can be sensed. In Section 2.4 , it will be helpful to keep these categories to understand the source of the information they provide.

\subsection{What Sensors Are Available?}

Dozens of abstract sensor models will soon appear in this article. To emphasize that these are grounded in reality, some widely available sensors are shown in Figure 4. Consider this, in addition to Figure 2, as a market where we can easily obtain real, physical sensors that fulfill the expectations of the mathematical models in Section 3. For each sensor, consider its category from Section 2.3, which indicates the type of phenomenon causing the sensor reading. It is also helpful to imagine sensors as being simple, which directly produces an output through transduction (an example is the LDR of Figure 2(a)), or compound which may be composed of several simpler sensors and even computational components (an example is the GPS device of Figure 2(b)).

The sensors in the top row of Figure 4 cost under $\$ 20$ US. The contact sensor in Figure 4(a) is simply a mechanical switch that forms a circuit when a strong enough force is applied. In combination with a faceplate, this could let a robot know whether it is hitting a wall. The sonar shown in Figure 4(b) emits a high-pitched sound and uses the time that it takes for the sound to rebound from the wall to estimate directional distance. A cheap compass (Dinsmore 1490) is shown in Figure 4(c), which indicates 8 possible general directions. A microphone, such as the one 


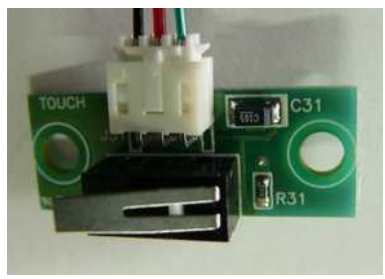

(a) Contact sensor

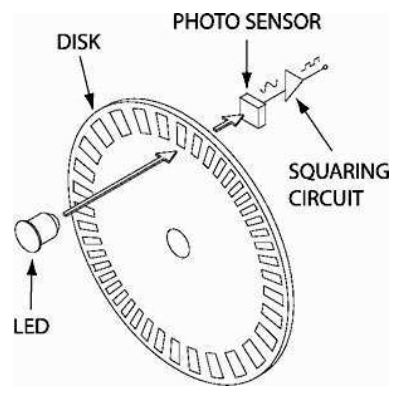

(e) Wheel encoder

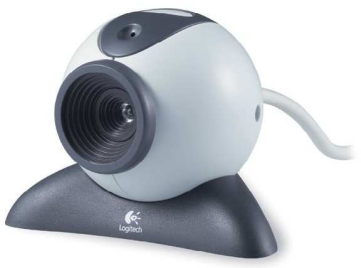

(i) Camera

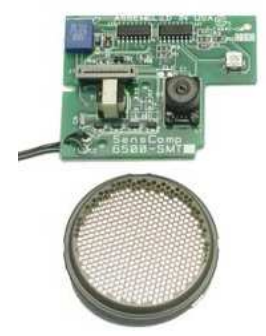

(b) Sonar

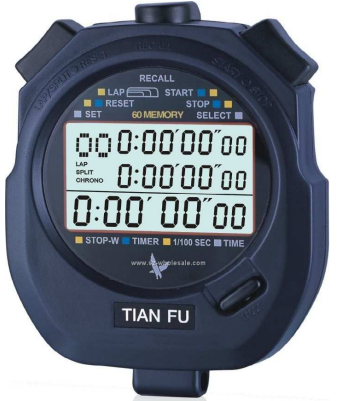

(f) Stopwatch/timer

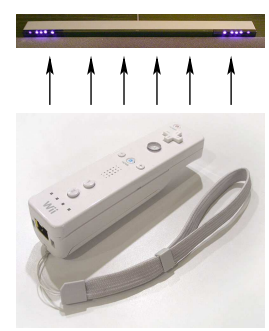

(j) Wii remote

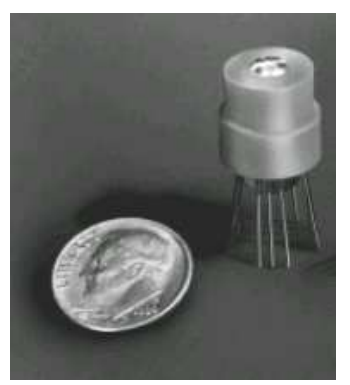

(c) Compass

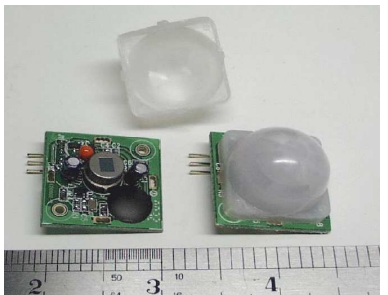

(g) Occupancy detector

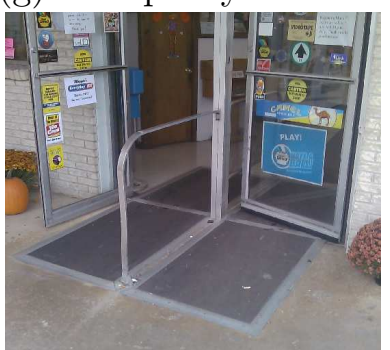

(k) Pressure mat

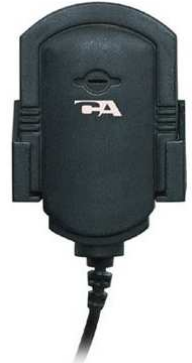

(d) Microphone

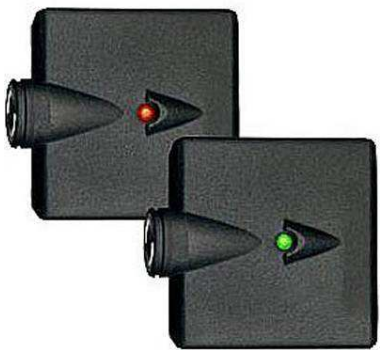

(h) Safety beam

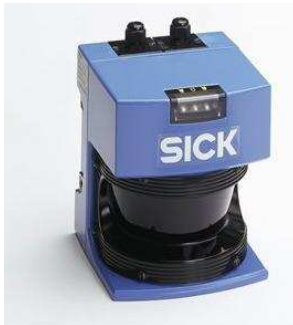

(l) SICK laser scanner

Figure 4: Some examples of widely available sensors, roughly sorted from low-cost to high-cost.

in Figure 4(d), can be used as a sensor in a wide variety of ways, from simple sound detection to sophisticated voice recognition.

Figure 4(e) depicts the inside of a wheel encoder, which is used in many applications to count wheel revolutions. By counting the number of light pulses of the LED visible through the disc holes, the total angle can be estimated. Figure 4(f) shows a stopwatch, which is just one kind of clock or chronometer that can be used to estimate time information (either the current time or total elapsed time). The sensors in Figures 4(g) and 4(h) are both based on infrared light detection. Figure 4(g) shows an example of a cheap occupancy detector (or motion detector), and Figure 4(h) shows a beam sensor, which is designed to keep a garage door from closing on someone or something.

Figure 4(i) shows a camera, which in combination with image processing or computer vision techniques, can perform a wide variety of functions, such as identifying people, tracking motion, analyzing lighting conditions, and so on. Figure $4(\mathrm{j})$ shows the Wii remote and its sensor bar, which are used in combination by the Nintendo Wii game console to infer hand motions and positions. A cheap camera tracks LEDs on the sensor bar to estimate position and orientation, and accelerometers in the remote estimate velocities. Figure $4(\mathrm{k})$ shows a pressure mat that sends a signal to open the door when someone steps on it. For all of the sensors shown so far, there are 
versions available for under $\$ 50$ US (some as low as $\$ 5$ ). In many settings, though, expensive sensors may be used to provide more complete information. The GPS device of Figure 2(b) is more complex and more expensive than the sensors shown so far in Figure 4. As a final example, however, consider the SICK laser rangefinder, which costs around $\$ 5000$ US and provides distance measurements at every half-degree over 180 degrees, with accuracy around one centimeter. Furthermore, a complete scan can be performed in about $1 / 30$ of a second. This sensor has been extremely popular over the last decade in mobile robotics for building indoor maps and localizing the robot.

Many other sensors are possible, such as mechanical scales to measure weight, gyroscopes to measure orientation, thermometers to measure temperature, radiation detectors, carbon monoxide detectors, and smoke alarms.

\subsection{Common Sensor Characteristics}

Most sensors are characterized in terms of a transfer function, which relates the possible inputs (phenomena) to the outputs (sensor readings). In Section 3, the important notion of a sensor mapping is introduced, which can be considered as a generalization and idealization of the transfer function. The transfer function is central in engineering manuals that characterize sensor performance $[17,52]$

Several important terms and concepts will be introduced with respect to the transfer function. For simplicity here, suppose that the transfer function is a mapping $g: \mathbb{R} \rightarrow \mathbb{R}$, and the sensor reading is $g(x)$ for some phenomenon $x$. Thus, the sensor transforms some real-valued phenomenon into a real-valued reading. The domain of $g$ may describe an absolute value or compare relative values. For example, a clock measures the absolute time and a chronometer measures the change in time.

The transfer function $g$ may be linear in simple cases, as in using a resistor to convert current into voltage; however, more generally it may be nonlinear. Since the so-called real numbers are merely a mathematical construction, the domain and range of $g$ are actually discrete in practice. The resolution of the sensor is indicated by the set of all possible values for $g(x)$. For example, a digital thermometer may report any value in the set $\{-20,-19, \ldots, 39,40\}$ degrees Celsius. For a more complex example, a camera may provide an image of $1024 \times 768$ pixels, each with 24 -bit intensity values.

Whereas resolution is based on the range of $g$, sensitivity is based on the domain. What set of stimuli produce the same sensor reading? For example, for what set of actual temperatures will the digital thermometer read 18 degrees? To fully understand sensitivity in a general way, study the preimages of sensor mappings in Section 3.2.2. This leads to the fundamental source of uncertainty covered in this paper.

More uncertainty may arise, however, due to lack of repeatability. If the sensor used under the exact conditions multiple times, does it always produce the same reading? Calibration can eliminate systematic (or repeatable) errors to improve sensor accuracy. For example, suppose we have purchased a cheap digital thermometer that has good repeatability but is usually inaccurate by several degrees. We can use a high-quality thermometer (assumed to be perfect) to compare the readings and make a lookup table. For example, when our cheap thermometer reads 17 and the high-quality thermometer reads 14, we will assume for ever more that the actual temperature is 14 whenever the cheap thermometer reads 17 . The lookup table can be considered as a mapping that is composed with $g$ to compensate for the errors. As another example, a wristwatch is actually a chronometer that is trying to behave as an absolute time sensor. Via frequent calibration (setting the watch), we are able to preserve this illusion. 


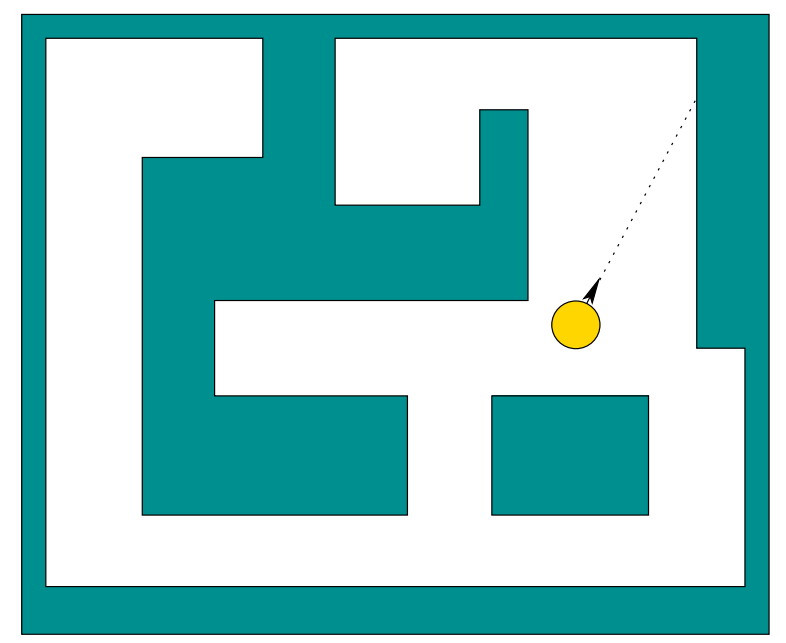

Figure 5: A mobile robot is placed in an indoor environment with polygonal walls. It measures the distance to the wall in the direction it is facing.

\section{Virtual Sensors}

Now that physical sensors have been described, we turn to making mathematical models of the information that is obtained from them. This leads to virtual sensors that could have many alternative physical implementations. The key idea in this section is to understand how two spaces are related:

1. The physical state space, in which each physical state is a cartoon-like description of the possible world external to the sensor.

2. The observation space, which is the set of possible sensor output values or observations.

We will define a sensor mapping, which indicates what the sensor is supposed to observe, given the cartoon-like description of the external world. For most sensors, a tremendous amount of uncertainty arises because the sensor does not observe everything about the external world. Understanding how to model and manipulate this uncertainty is the main goal of this section. Additional uncertainty may arise due to sensor noise or calibration errors, but it is important to consider these separately. Eventually, all sources of uncertainty combine, making it difficult or impossible to reason about them without understanding them independently.

\subsection{Physical State Spaces}

Consider the scenario shown in Figure 5, in which an indoor mobile robot measures the distance to the wall in the direction that it happens to be facing. This could, for example, be achieved by mounting a sonar or laser on the front of the robot. If the sensor is functioning perfectly and reads 3 meters, then what do we learn about the external world? This depends on what is already known before the sensor observation. Do we already know the robot's configuration (position and orientation)? Do we have a precise geometric map of all of the walls? If we know both of these already, then we would learn absolutely nothing from the sensor observation. If we know the robot's configuration but do not have a map, then the sensor reading provides information about how the walls are arranged. Alternatively, if we have a map but not the configuration, then we learn something about the robot's position and orientation. If we have neither, then something is 
learned about both the configuration and the map of walls. In robotics, this learning is referred to as simultaneous localization and mapping (SLAM). The purpose of defining the physical state space is to characterize the set of possible external worlds that are consistent with a sensor observation and whatever background information is given.

Since the physical state contains both configuration and map information, a common structure frequently appears for the physical state space. Let $\mathcal{Z}$ be any set of sets. Each $Z \in \mathcal{Z}$ can be imagined as a "map" of the world and each $z \in Z$ would be the configuration or "place" in the map. If the configuration and map are unknown, then the state space would be the set of all $(z, Z)$ such that $z \in Z$ and $Z \in \mathcal{Z}$.

\subsubsection{A mobile robot among obstacles}

No walls Return to Figure 5. If there were no walls, then the robot could move to any position $\left(q_{x}, q_{y}\right) \in \mathbb{R}^{2}$ and orientation $q_{\theta} \in[0,2 \pi)$. The physical state, denoted by $x$, is completely expressed by $x=\left(q_{x}, q_{y}, q_{\theta}\right)$. The physical state space, denoted by $X$, in this case is the set of all robot positions and orientations. We can imagine $X \subset \mathbb{R}^{3}$ by noting that $q_{y}, q_{y} \in \mathbb{R}$ and $q_{\theta} \in[0,2 \pi) \subset \mathbb{R}$. This will be perfectly fine for defining a sensor; however, we sometimes need to capture additional structure. Here, the fact that 0 and $2 \pi$ are the same orientation has not been taken into account. Formally, we can place the robot at the origin, facing the $x$ axis, and apply homogeneous transformation matrices to translate and rotate it $[34,37]$. The set of all such transformations is called a matrix group. In particular, we obtain $X=S E(2)$, which is the set of all 3 by 3 matrices that can translate and rotate the robot. As is common in robotics, we could alternatively write $X=\mathbb{R}^{2} \times S^{1}$, in which $S^{1}$ denotes a circle in the topological sense and represents the set of possible orientations. Let $S^{1}=[0,2 \pi]$ with a declaration that 0 and $2 \pi$ are the same. These issues are quite familiar in robotics, control theory, and classical mechanics; see $[1,6,45,46]$. The discussion in this paper will be kept simple to avoid technicalities that are mostly orthogonal to our subject of interest.

Known map Now suppose that the robot has a perfect polygonal map of its environment. This constrains the robot position $\left(q_{x}, q_{y}\right)$ to lie in some set $E \subset \mathbb{R}^{2}$ that has a polygonal boundary. The state space becomes $X=E \times S^{1}$, in which $S^{1}$ once again accounts all possible orientations.

One of several maps The robot is now told that one of $k$ possible maps is the true one. For example, we may have a set $\mathcal{E}$ of five possible maps $\left\{E_{1}, E_{2}, E_{3}, E_{4}, E_{5}\right\}$. This can be imagined as having $k=5$ copies of the previous state space. The state space $X$ is the set of all pairs $\left(q, E_{i}\right)$ in which $q=\left(q_{x}, q_{y}, q_{\theta}\right),\left(q_{x}, q_{y}\right) \in E_{i}$, and $E_{i} \in \mathcal{E}$.

Unknown map If the map is completely unknown, then the robot may be told only the map family, which is an infinite collection. For example, $\mathcal{E}$ may be the set of all polygonal subsets ${ }^{1}$ of $\mathbb{R}^{2}$. Every map can be specified by a polygon and describes a subset of $\mathbb{R}^{2}$. The state space $X$ is the set of all pairs $(q, E)$ in which $\left(q_{x}, q_{y}\right) \in E$ and $E \in \mathcal{E}$. Note that we can write $X \subset S E(2) \times \mathcal{E}$.

Numerous other map families can be made. Here are several thought-provoking possibilities, in which each defines $\mathcal{E}$ as a set of subsets of $\mathbb{R}^{2}$. Thus, $\mathcal{E}$ could be:

- The set of all connected, bounded polygonal subsets that have no interior holes (formally, they are simply connected).

\footnotetext{
${ }^{1}$ To be more precise, each subset must be closed, bounded, and simply connected.
} 


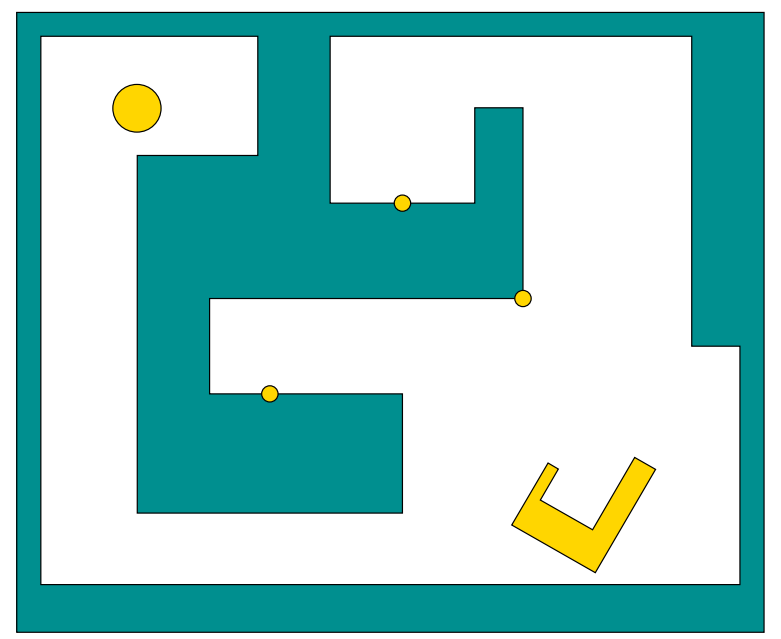

Figure 6: The environment may contain various kinds of bodies, such as robots, landmarks, objects, pebbles, targets, obstacles, evaders, treasures, and towers.

- The previous set expanded to include all cases in which the polygonal region has a finite number of polygonal holes.

- All subsets of $\mathbb{R}^{2}$ that have a finite number of points removed.

- All subsets of $\mathbb{R}^{2}$ that can be obtained by removing a finite collection of nonoverlapping discs.

- All subsets of $\mathbb{R}^{2}$ obtained by removing a finite collection of nonoverlapping convex sets.

- A collection of piecewise-analytic subsets of $\mathbb{R}^{2}$.

Each map does not even have to contain homogeneous components. For example, each could be described as a polygonal region that has a finite number of interior points removed. Furthermore, three-dimensional versions exist for the families above. For example, $\mathcal{E}$ could be a set of polyhedral regions in $\mathbb{R}^{3}$.

In some of the examples above, obstacles such as points or discs are removed. We could imagine having an augmented map in which a label is associated with each obstacle. For example, if $n$ disks are removed, then they may be numbered from 1 to $n$. This becomes a special case of the models considered next.

\subsubsection{A bunch of bodies}

Now consider placing other kinds of entities into an environment $E$, which may or may not contain robots. Each such entity will be called a body, which could have one of a number of possible interpretations in practice. A body $B$ occupies a subset of $E$ and can be transformed using its own configuration parameters. For example, a body could be a point that is transformed by $\left(q_{x}, q_{y}\right)$ parameters or a rectangle that is transformed by $\left(q_{x}, q_{y}, q_{\theta}\right)$ parameters. We can write $B\left(q_{x}, q_{y}, q_{\theta}\right) \subset E$ to indicate the set of points occupied by $B$ when at configuration $\left(q_{x}, q_{y}, q_{\theta}\right)$. In general, bodies could be as complex as any robots considered in robot motion planning; however, this is too much of a digression for this paper; see [34,37] for understanding how the configuration space of bodies is constrained when they are not points. Here, it will be assumed that all bodies are points, except for obstacles. 
In this article, bodies may have many different interpretations and uses. Here are terms and examples that appear all over the literature:

- Robot: A body that carries sensors, performs computations, and executes motion commands.

- Landmark: Usually a small body that has a known location and is easily detectable and distinguishable from others.

- Object: A body that can be detected and manipulated by a robot. It can be carried by a robot or dropped at a location.

- Pebble: A small object that is used as a marker to detect when a place has been revisited.

- Target: A person, a robot, or any other moving body that we would like to monitor using a sensor.

- Obstacle: A fixed or moving body that obstructs the motions of others.

- Evader: An unpredictable moving body that attempts to elude detection.

- Treasure: Usually a stationary body that has an unknown location but is easy to recognize by a sensor directly over it.

- Tower: A body that transmits a signal, such as a cell-phone tower or a lighthouse.

Rather than worry about particular names of bodies, which are clearly arbitrary, it is more important to think about their mathematical characteristics. Think about these three important properties of a body:

1. What are its motion capabilities?

2. Can it be distinguished from other bodies?

3. How does it interact with other bodies?

First consider motion capabilities. At one extreme, a body could be static, which means that it never moves. Its configuration could nevertheless be unknown. If the body moves, then it may have predictable or unpredictable motion. Furthermore, the body may be able to move by itself, as in a person, or it may move only when manipulated by other bodies, such as a robot pushing a box.

Next we handle distinguishability. Consider a collection of bodies $B_{1}, \ldots, B_{n}$ that are distinguishable simply by the fact that each is uniquely defined. We can now define any equivalence relation $\sim$ and say $B_{i} \sim B_{j}$ if and only if they cannot be distinguished from each other. Another way to achieve this is by defining a set of labels and assigning a not-necessarily-unique label to each body. For example, the bodies may be people, and we may label them as male and female. More complicated models are possible, but are not considered here. (For example, indistinguishability does not even have to be an equivalence relation: Perhaps $B_{i}$ and $B_{j}$ are pairwise indistinguishable, $B_{j}$ and $B_{k}$ are pairwise indistinguishable, but $B_{i}$ and $B_{k}$ could be distinguishable.)

Finally, think about how bodies might interact or interfere with each other. Three interaction types are generally possible between a pair $B_{1}, B_{2}$, of bodies:

- Sensor obstruction: Suppose a sensor would like to observe information about body $B_{1}$. Does body $B_{2}$ interfere with the observation? For example, a truck could block the view of a camera, but a sheet of glass might not. 


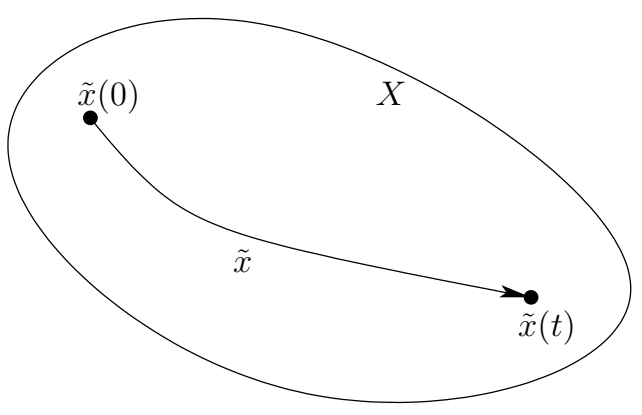

Mapping into $X$

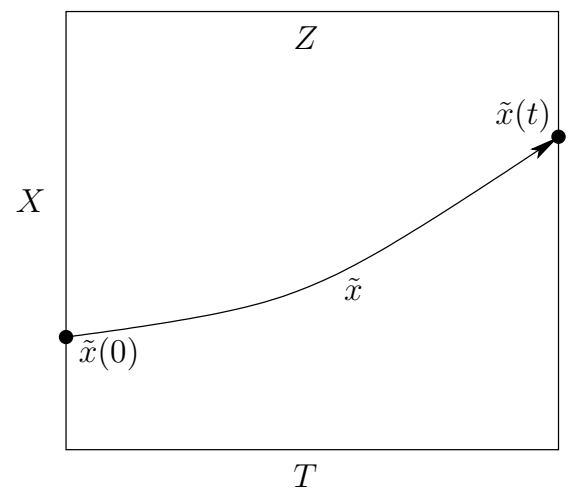

Mapping into $Z$

Figure 7: A trajectory can be viewed as a time-parametrized path through $X$ or a one-dimensional set that traverses $Z$ from left to right.

- Motion obstruction: Does body $B_{2}$ obstruct the possible motions of body $B_{1}$ ? If so, then $B_{2}$ becomes an obstacle that must be avoided.

- Manipulation: In this case, body $B_{1}$ could cause body $B_{2}$ to move. For example, if $B_{2}$ is an obstacle, then $B_{1}$ might push it out of the way.

In the remainder of the paper, many different kinds of bodies will appear and it is crucial to pay attention to their properties rather than their particular names. In all cases, it will be assumed that bodies are contained in $E$.

\subsubsection{Introducing Time}

Of course the world is not static. If the physical state space $X$ is meant to be a cartoon-like description of the world, it represents only a single snapshot. Time will now be introduced to animate the world. Let $T$ refer to an interval of time, in which the most convenient case is $T=[0, \infty)$. Starting from any physical state space $X$ as defined above, we can obtain a state-time space $Z=X \times T$, in which each $z \in Z$ is a pair $z=(x, t)$ and $x$ is the state at time $t$.

Since time always marches forward, we can consider the "animation" as a path through $Z$ that is parametrized by time. This leads to a state trajectory, $\tilde{x}: T \rightarrow X$. The value $\tilde{x}(t) \in X$ represents the state at time $t$. The value $\tilde{x}(0)$ is called the initial state. See Figure 7 .

The configurations of bodies may change over time, but are continuous functions. In fact, they are usually differentiable, leading to time derivatives. For example $\dot{q}=d q / d t$ is velocity and $\ddot{q}=d^{2} q / d t^{2}$ is acceleration. Such quantities can be incorporated directly into the state to expand $X$ into a phase space as considered in mechanics. For example, $x=(q, \dot{q})$ is the phase of a mechanical system in Lagrangian mechanics. However, the rest of this paper will avoid working directly with velocities.

Before time was introduced, $\mathcal{E}$ was introduced to represent possible maps. Now it is possible that the maps vary over time, along with configurations. This variation may or may not be predictable.

\subsection{Virtual Sensor Models}

Now that the state space $X$ is defined, we can introduce numerous sensor models that are inspired by the physical sensors in Section 2.4, but are expressed abstractly in terms of $X$. 

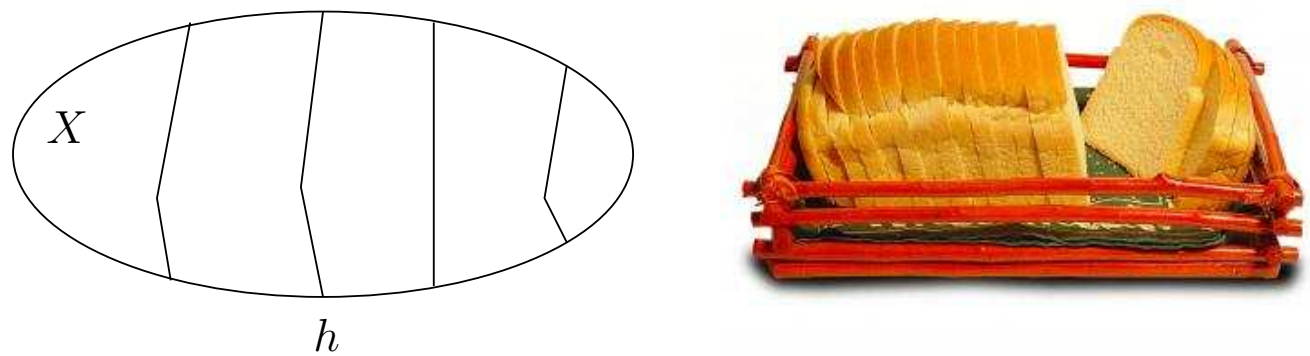

Figure 8: (a) A sensor should be viewed as a gadget that partitions the physical state space $X$. (b) A sensor definition is much like running $X$ through a bread slicer. Each "slice" is a preimage.

\subsubsection{The Sensor Mapping}

We define models of instantaneous sensors, which use the physical state to immediately produce an observation. Let $X$ be any physical state space. Let $Y$ denote the observation space, which is the set of all possible sensor observations. A virtual sensor is defined by a function

$$
h: X \rightarrow Y,
$$

called the sensor mapping, which is very much like the transfer function described in Section 2.5. The interpretation is that when $x \in X$, the sensor instantaneously observes $y=h(x) \in Y$. Equation 1 is the most important definition in this paper.

\subsubsection{Preimages: The amount of state uncertainty due to a sensor}

What does an observation $y$ tell us about the external, physical state? To understand this, we should think about all states $x \in X$ that could have produced the observation. For a given sensor mapping $h$ this is defined as

$$
h^{-1}(y)=\{x \in X \mid y=h(x)\},
$$

and is called the preimage of $y$. If $h$ were invertible, then $h^{-1}$ would represent the inverse; however, because our sensor models are usually many-to-one mappings, $h^{-1}(y)$ is a subset of $X$, which yields all $x$ that map to $y$.

Consider the collection of subsets of $X$ obtained by forming $h^{-1}(y)$ for every $y \in Y$. These sets are disjoint because a state $x$ cannot produce multiple observations. Since $h$ is a function on all of $X$, the collection of subsets forms a partition of $X$. For a given sensor mapping $h$, the corresponding partition is denoted as $\Pi(h)$.

The connection between $h$ and $\Pi(h)$ is fundamental to sensing; see Figure 8. As soon as $X$, $Y$, and a sensor mapping are defined, you should immediately think about how $X$ is partitioned. The sets in $\Pi(h)$ can be viewed as equivalence classes. For any $x, x^{\prime} \in X$, equivalence implies that $h(x)=h\left(x^{\prime}\right)$. These states are indistinguishable when using the sensor. In an intuitive way, $\Pi(h)$ gives the sensor's sensitivity to states, or the "resolution" at which the state can be observed. The equivalence classes are the most basic source of uncertainty associated with a sensor.

Numerous virtual sensor models will now be defined in terms of (1). Consider partitions of $X$ induced by each one. The sensor models can be physically implemented in several alternative ways using various sensors. If (1) seems too idealistic, considering that sensors may be unpredictable, 
do not worry. Sensor disturbances and other complications are handled in Section 3.4. In addition, sensors based on measuring vector fields through the environment are covered in the appendix.

\subsubsection{Basic Examples}

Models 1, 2 and 3 will be useful for comparisons to other, more practical models.

Model 1 (Dummy Sensor)

At one extreme, a worthless sensor can be made by letting $Y=\{0\}$ with $h(x)=0$ for all $x \in X$. This sensor never changes its output, thus providing no information about the external world. The preimage $h^{-1}(y)=X$ for any $y \in Y$. The induced partition $\Pi(h)$ is the coarsest possible.

Model 2 (Identity Sensor)

At the other extreme, we can define an "all knowing" sensor by setting $Y=X$ and letting $y=h(x)=x$. From a single observation, no uncertainty about the external world exists. In this case, $h^{-1}(y)=\{x\}$ and $\Pi(h)$ is the finest partition possible of $X$. All elements are singletons.

Model 3 (Bijective Sensor)

Let $h$ be any bijective function from $X$ to $Y$. By one interpretation, this sensor is as powerful as Model 2 (identity) because $x$ can be reconstructed from $y$ using the inverse of $h$. In practice, however, it may be costly or impossible to compute the inverse of $h$. Again, all preimages are singletons and the finest partition possible of $X$ is obtained.

The next two models are generic but useful in many settings.

Model 4 (Linear Sensor)

For a model that is in between the power of Models 1 (dummy) and 2 (identity), suppose $X=Y=$ $\mathbb{R}^{3}$. Let $y=h(x)=C x$ for some 3 by 3 real-valued matrix $C$. In this case, $x$ can be reconstructed from $y$ if $C$ has full rank. This is a special case of Model 3 (bijective). More generally, if $C$ has rank $k \in\{1,2,3\}$, then there is a $(3-k)$-dimensional linear subspace of $X$ that produces the same observation $y$. Linear sensors can be similarly defined for any $X=\mathbb{R}^{n}$ and $Y=\mathbb{R}^{m}$. In fact, this is the standard output model for linear systems in control theory [7]. The preimages of $h$ are hyperplanes in $\mathbb{R}^{n}$.

Model 5 (Projection Sensor)

This convenient sensor directly observes some components of $X$. For example, if $x=\left(x_{1}, x_{2}, x_{3}\right) \in$ $\mathbb{R}^{3}$, then a projection sensor could yield the first two coordinates. In this case, we have $Y=\mathbb{R}^{2}$ and $y=h(x)=\left(x_{1}, x_{2}\right)$. The preimages in this case are subspaces of $X$ in which the unobserved coordinates are free.

\subsubsection{Depth Sensors}

We now introduce an important family of sensor models that arise in mobile robotics. Using the state space models from Section 3.1.1, depth sensors base the observation on distance from the sensor to the boundary of $E$. The state space is $X \subset S E(2) \times \mathcal{E}$, in which each state $x \in X$ 


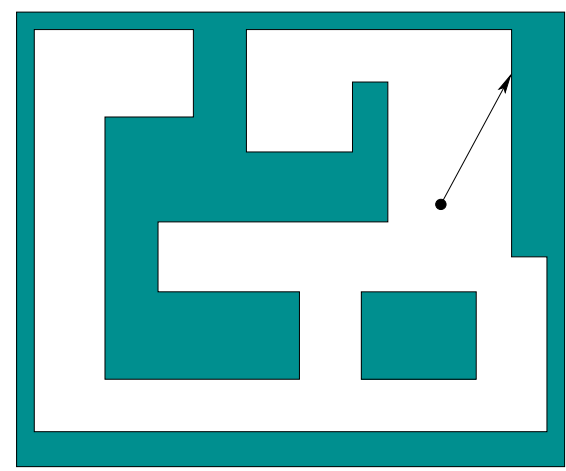

(a) Directional depth

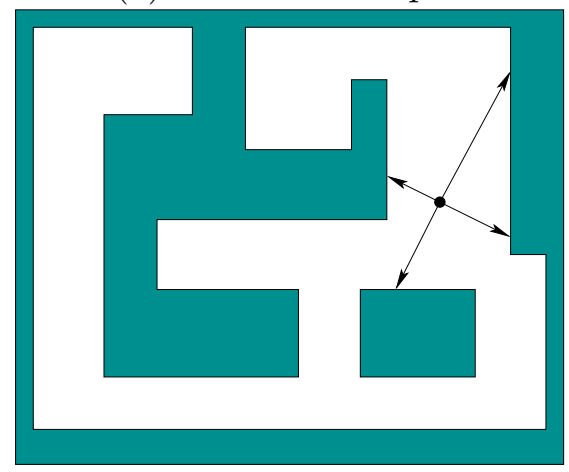

(c) K-directional depth

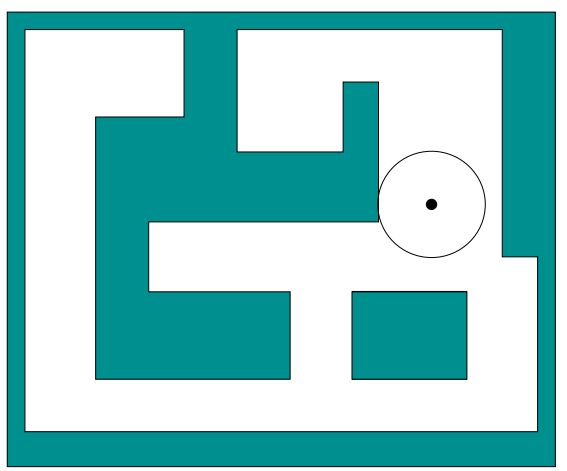

(b) Boundary distance

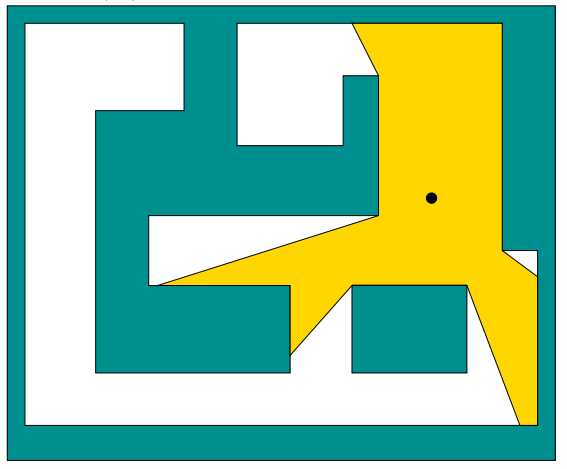

(d) Omnidirectional depth

Figure 9: Several variations exist for depth sensors.

is represented as $x=\left(q_{x}, q_{y}, q_{\theta}, E\right)$ with $\left(q_{x}, q_{y}\right) \in E$ and $E \in \mathcal{E}$. For convenience, the notation $p=\left(q_{x}, q_{y}\right)$ and $\theta=q_{\theta}$ will be used.

Model 6 (Directional Depth Sensor)

How far away is the wall in the direction the robot is facing? Figure 9(a) shows a mobile robot facing a direction to the upper right. Let $b(x)$ denote the point on the boundary of $E$ that is struck by a ray emanating from $p$ and extended in the direction of $\theta$. The sensor mapping

$$
h_{d}(p, \theta, E)=\|p-b(x)\|
$$

precisely yields the distance to the wall. This could be implemented using a sonar, shown in Figure 4 (b), or a single laser/camera combination.

Model 7 (Boundary Distance Sensor)

How far away is the nearest wall, regardless of direction? As shown in Figure 9(b), this can be considered as the radius of the largest disk that can be placed in $E$, centered on the robot. The sensor mapping can be expressed in terms of $h_{d}$ :

$$
h_{b d}(p, \theta, E)=\min _{\theta^{\prime} \in[0,2 \pi)} h_{d}\left(p, \theta^{\prime}, E\right)
$$

Note that $h_{b d}$ ignores $\theta$, as expected. This sensor could be implemented expensively by using two SICK laser scanners (shown in Figure 4(l)) and reporting the minimum distance value. A cruder version could be made from an array of sonars. 
Model 8 (Proximity Sensor)

Imagine that a light goes on when the robot is within a certain distance, $\epsilon>0$, to the wall. This is easily modeled as

$$
h_{p \epsilon}(p, \theta, E)= \begin{cases}1 & \text { if } h_{b d}(p, \theta, E) \leq \epsilon \\ 0 & \text { otherwise }\end{cases}
$$

An array of simple infrared sensors could accomplish this. A directional version could alternatively be made by using $h_{d}$ from (3) instead of $h_{b d}$ above.

Model 9 (Boundary Sensor)

By reducing $\epsilon$ to 0 , we obtain a sensor that indicates whether the robot is touching the boundary. This is called a boundary or contact sensor:

$$
h_{b d}(p, \theta, E)= \begin{cases}1 & \text { if } h_{b d}(p, \theta, E)=0 \\ 0 & \text { otherwise. }\end{cases}
$$

Note that $h_{b d}(p, \theta, E)=h_{p 0}(p, \theta, E)$. Again, a directional version can be made by substituting $h_{d}$ for $h_{b d}$. This sensor could be implemented using contact sensors, as shown in Figure 4(a).

Model 10 (Shifted Directional Depth Sensor)

This model is convenient for defining the next two. It is simply a directional sensor that allows an offset angle $\phi$ between the direction that the robot faces and the direction that the sensor is pointing:

$$
h_{s d \phi}(p, \theta, E)=\|p-b(p, \theta+\phi, E)\| .
$$

In comparison to $h_{d}$ in $(3)$, only $\phi$ has been inserted.

Model 11 (K-Directional Depth Sensor)

Suppose there is a set of offset angles $\phi_{1}, \ldots, \phi_{k}$, which in most cases are regularly spaced. Figure 9 (c) shows an example for which $k=4$ and the directions are spaced at right angles. In this case, the observation is a vector $y=\left(y_{1}, \ldots, y_{k}\right)$ in which

$$
y_{i}=h_{i}(p, \theta, E)=h_{s d \phi_{i}}(p, \theta, E) .
$$

This sensor could be implemented by an array of evenly spaced sonars, fixed around the boundary of a round robot. A laser scanner provides yet another implementation.

Model 12 (Omnidirectional Depth Sensor)

In the limiting case, imagine letting $k$ become infinite so that measurements are taken in all directions, as shown in Figure 9(d). In this case, the observation is an entire function (imagine an infinite-dimensional vector). We obtain $h_{o d}(x)=y$, in which $y: S^{1} \rightarrow[0, \infty)$ and

$$
y(\phi)=h_{o d \phi}(p, \theta, E) .
$$

This means that evaluating the function $y$ at $\phi \in[0,2 \pi)$ yields the shifted directional distance $h_{o d \phi}(p, \theta, E)$; see Figure 10. In practice, most sensors have a limited range of directions. In this case the domain of $y$ can be restricted from $S^{1}$ to $\left[\phi_{\min }, \phi_{\max }\right]$ to obtain observations of the form $y:\left[\phi_{\min }, \phi_{\max }\right] \rightarrow[0, \infty)$. In practice, this corresponds closely to the dense measurements obtained from the SICK laser scanner, shown in Figure 4(l). That one scans over 180 degrees; however, 360-degree variants exist. 


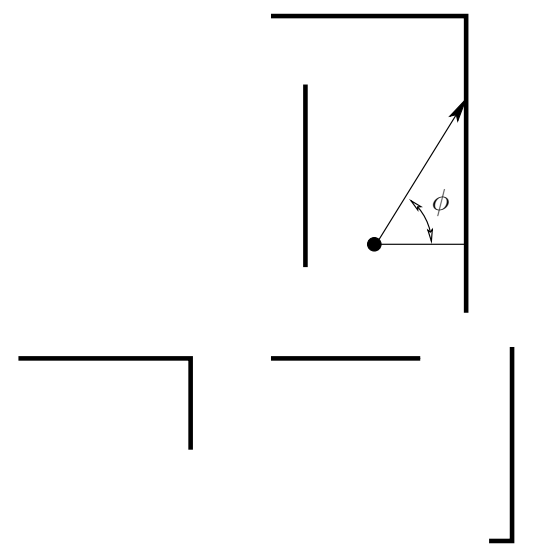

Figure 10: For the omnidirectional depth sensor, Model 12 a function $y: S^{1} \rightarrow[0, \infty)$ is obtain in which each $y(\phi)$ is the depth in the direction $\theta+\phi$. The figure shows how the depth data appears for the environment in Figure $9(\mathrm{a})$ and $\theta=0$.

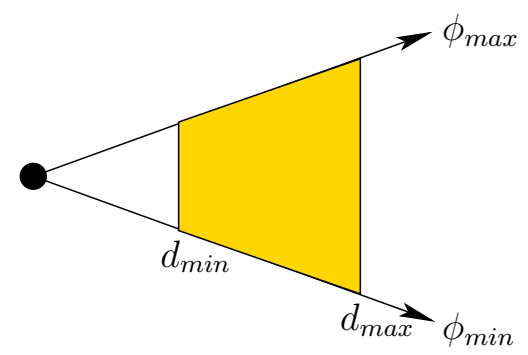

Figure 11: A depth sensor that has limited range and angle.

For all of the sensor models from Model 6 to 12, an important depth-limited variant can be made. When placed into large enough environments, a sensor might not be able to detect a wall that is too far away. Instead of a distance range $[0, \infty)$, we could have a range of distances from $d_{\min }$ to $d_{\max }$. The following model illustrates the idea.

Model 13 (Depth-Limited Directional Depth Sensor)

Model 6 (directional depth) can be modified to obtain a depth-limited version in which the sensor cannot give measurements when the distance is outside of the interval $\left[d_{\min }, d_{\max }\right]$ for some $d_{\min }, d_{\max } \geq 0$ with $d_{\min }<d_{\max }$. Let $d(x)=p-b(x)$. The sensor mapping is

$$
h_{d d}(p, \theta, E)= \begin{cases}d(x) & \text { if } d_{\min } \leq d(x) \leq d_{\max } \\ \# & \text { otherwise }\end{cases}
$$

in which the symbol \# indicates that the sensor cannot determine the distance. If the wall is too far away, most sensors will not report a value. For example, a sonar echo will not be heard. Thus, this model is realistic in many settings.

Figure 11 shows how a sensor that is both depth limited and angle limited might appear. Section 3.2.5 covers many depth-limited sensors, but with a different purpose. Rather than measuring depth, they are designed to detect bodies within their field of view. Depth-limited sensors also become important in Section 3.2.7, for defining gap sensors. 


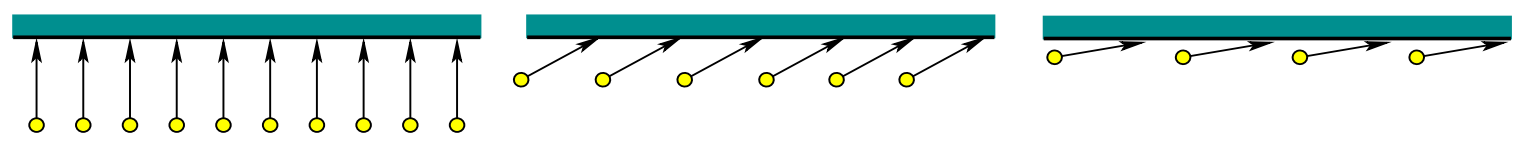

Figure 12: The preimage for a single-directional depth sensor is a two-dimensional subset of $S E(2)$, assuming the environment is given. Shown here are several robot configurations within the same preimage.

Now think about the preimages associated with the various depth senors and the resulting partition $\Pi(X)$. First consider the case of a given polygonal environment, leading to $X=E \times$ $S^{1}$. For Model 6 (directional depth), each $h^{-1}(y)$ is generally a two-dimensional subset of $X$ that corresponds to all possible configurations from which the same directional distance could be obtained. Thus, $\Pi(h)$ is a collection of disjoint, two-dimensional subsets of $E \times S^{1}$. For example, equivalent states along a single wall are depicted in Figure 12. Using the boundary sensor, Model 9, $\Pi(h)$, contains only two classes: All states in which the robot is in the interior of $E$, and all states in which it is on the boundary of $E$. The omnidirectional depth sensor, Model 12, is quite powerful. This leads to very small preimages. In most cases, these correspond to the finite set of symmetry classes of the environment. Such symmetries are usually encountered in robot localization. For example, in the environment at the extreme left of Figure 13, $h^{-1}(y)$ is a threeelement set, corresponding to the three possible orientations at which the same observation could be obtained.

Now suppose that the environment is unknown, leading to $X \subset S E(2) \times \mathcal{E}$. Each $h^{-1}(y)$ contains a set of possible environment and robot configuration pairs that could have produced the observation. In the case of a boundary sensor, $h^{-1}(1)$ would mean "all environments and configurations in which the robot is touching a wall". For the omnidirectional sensor, $h^{-1}(y)$ indicates all ways that the environment could exist beyond the field of view of the sensor.

\subsubsection{Detection Sensors}

As the name suggests, this family models sensors that detect whether one of more bodies are within their sensing range. Physical examples include a camera, the occupancy detector of Figure 4(e), and the pressure mat of Figure $4(\mathrm{k})$.

Three fundamental aspects become important in detection sensor models:

1. Can the sensor move? For example, it could be mounted on a robot or it could be fixed to a wall.

2. Are the bodies so large relative to the range of the sensor that the body models cannot be simplified to points?

3. Can the sensor provide additional information that helps to classify a body within its detection region?

If the answer is "no" to all three questions, then the simplest case is obtained: A stationary detection sensor that indicates whether at least one point body is within its range. For this case, let $V \subset E$ be called the detection region. Suppose that $E$ contains one ore more point bodies that can move around. Note that $V$ can be any shape, as shown in Figure 13.

We now present several models, starting with the simplest case and eventually taking into account all three complications above. 


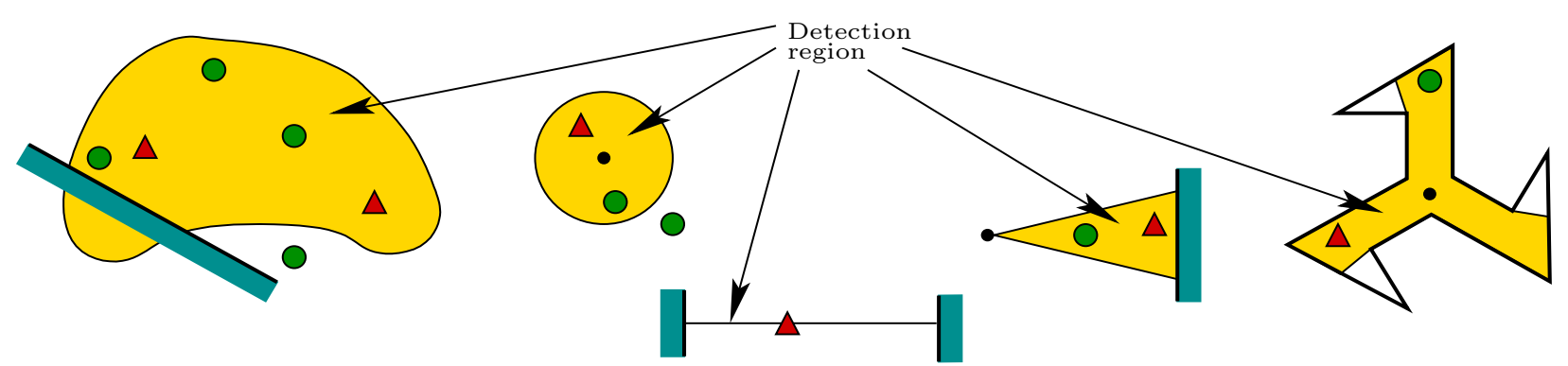

Figure 13: A detection region is a subset of the environment in which moving bodies (shown as triangles and discs) are detected. The detection regions may appear in various shapes and may or may not be attached to a movable body.

Model 14 (Static Binary Detector)

A simple detection model can now be defined in terms of $V$. Suppose that a single body moves in $E$ and its position is denoted by $p$. The sensor mapping is

$$
h(p, E)= \begin{cases}1 & \text { if } p \in V \\ 0 & \text { otherwise }\end{cases}
$$

It simply indicates whether the body is in the detection region. Physically, this could correspond to a cheap occupancy sensor that is mounted on the wall.

There are three separate axes along which to generalize (11). Each will be handled separately, but all three generalizations can clearly be combined.

Model 15 (Moving Binary Detector)

Suppose the sensor can move, as in a camera that is mounted on a mobile robot. Let $q$ denote the configuration of the body that is carrying the sensor. We now obtain $V(q) \subset E$ as the configurationdependent detection region. The sensor mapping is

$$
h(p, E)= \begin{cases}1 & \text { if } p \in V(q) \\ 0 & \text { otherwise }\end{cases}
$$

\section{Model 16 (Detecting Larger Bodies)}

What if the body has some shape and is transformed by $q^{\prime}$ to obtain $B\left(q^{\prime}\right) \subset E$ ? Then we could, for example, make a static binary detector for general bodies:

$$
h\left(q^{\prime}, E\right)= \begin{cases}1 & \text { if } B\left(q^{\prime}\right) \cap V \neq \emptyset \\ 0 & \text { otherwise. }\end{cases}
$$

The sensor detects the body if any part of it enters $V$. This is similar to the definition of configuration-space obstacle region, $\mathcal{C}_{o b s}$, in motion planning $[8,34,37]$. See Figure 14 . An alternative definition would require the body to be contained in the detection region: $B\left(q^{\prime}\right) \subseteq V$. If the sensor can additionally move, then $V$ in (13) is replaced with $V(q)$ and the state becomes $x=\left(q, q^{\prime}, E\right)$. 


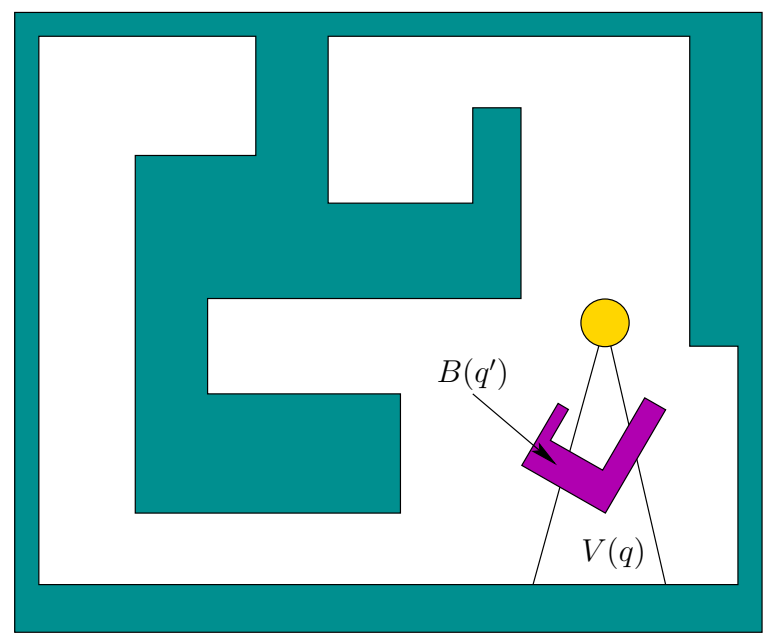

Figure 14: For larger bodies, we must declare either some or all of the body must be in view to be detected.
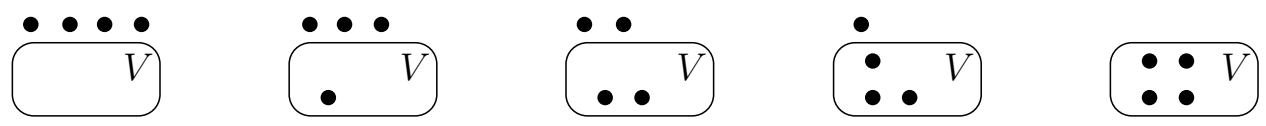

Figure 15: A fixed detection sensor among 4 moving points in $\mathbb{R}^{2}$ yields these 5 equivalences classes for the partition $\Pi(h)$ of $X$. In this model, the observation $y$ is the number of points in $V$.

Now suppose there are multiple bodies. Let $P=\left\{p_{1}, \ldots, p_{n}\right\}$ denote a set of $n$ point bodies that move in $E$. The state becomes $x=\left(q, p_{1}, \ldots, p_{n}, E\right)$ in which $q$ is the sensor configuration.

Model 17 (At-Least-One-Body Detector)

This model detects whether there is at least one body in the detection region $V(q)$. The sensor mapping is

$$
h\left(q, p_{1}, \ldots, p_{n}, E\right)= \begin{cases}1 & \text { if for any } i, p_{i} \in V(q) \\ 0 & \text { otherwise }\end{cases}
$$

Model 18 (Body Counter)

Moving away from a binary sensor, the sensor could count the number of bodies in the detection region $V(q)$. The sensor mapping is

$$
h\left(q, p_{1}, \ldots, p_{n}, E\right)=|P \cap V(q)|,
$$

in which $|\cdot|$ denotes the number of elements in a set.

A special case of Model 18 is presented next.

Model 19 (Planar Body Counter)

Suppose that $n$ point bodies move in $\mathbb{R}^{2}$ and a static detection sensor is installed that counts how many points are within a fixed detection region $V$. The state space is $X=\mathbb{R}^{2 n}$ and observation space is $Y=\{0,1, \ldots, n\}$. Now consider the preimages. The partition $\Pi(h)$ is formed by $n+1$ 
equivalence classes. Figure 15 shows how these subsets of $X$ could be depicted for the case of $n=4$. If the sensor were additionally able to distinguish between the points and determine which are in $V$, then there would be $2^{n}$ equivalence classes. Such a sensor would be strictly more powerful and the equivalence classes would be correspondingly smaller.

More generally, we can consider bodies that are partially distinguishable to the sensor. Let $L$ be a set of class labels, attribute values, or feature values that can be assigned to bodies, as discussed in Section 3.1.2. Let $\ell$ be an assignment mapping $\ell:\{1, \ldots, n\} \rightarrow L$.

Model 20 (Labeled-Body Detector)

Suppose that we want to detect when a body is in the detection region and it has a particular label $\lambda \in L$. In this case, the sensor mapping is:

$$
h_{\lambda}(p, E)= \begin{cases}1 & \text { if for some } i, p_{i} \in V \text { and } \ell(i)=\lambda \\ 0 & \text { otherwise. }\end{cases}
$$

In a physical implementation, a camera could be used with computer vision techniques to classify and label bodies in the image.

Numerous other extensions and variations are possible. Here are some ideas: 1) a detection sensor could count bodies that share the same label, 2) each body could be modeled as having its own configuration parameters, to allow translation and rotation, 3) the number of bodies may not be specified in advance, 4) if the boundary of $V$ has multiple components, the sensor might indicate which component was crossed, and 5) multiple detection sensors could be in use, each of which classifying bodies differently.

\subsubsection{Relational Sensors}

We now take detection sensors as a starting point and allow them to provide a critical piece of information: How is one body situated relative to another? This leads to the family of relational sensors, a term introduced by Guibas [22]. A detection sensor only tells us which bodies are in view, whereas a relational sensor additionally indicates how they are arranged.

Let $R$ be any relation on the set of all bodies. For a pair of bodies, $B_{1}$ and $B_{2}$, examples of $R\left(B_{1}, B_{2}\right)$ are:

- $B_{1}$ is in front of $B_{2}$

- $B_{1}$ is to the left of $B_{2}$

- $B_{1}$ is on top of $B_{2}$

- $B_{1}$ is closer than $B_{2}$

- $B_{1}$ is bigger than $B_{2}$.

This information actually depends on the full state: The configurations of the sensor and the bodies. We therefore write the relation as $R_{x}$ and define it over the set $\{1, \ldots, n\}$, which includes the indices of the bodies. Using this notation for the "in front of" example, $R_{x}(i, j)$ means that body $B_{i}$ is in front of $B_{j}$ when viewed from the state $x=\left(q_{s}, q_{1}, \ldots, q_{n}\right)$, in which $q_{s}$ is the sensor configuration and each remaining $q_{i}$ is the $i$ th body configuration. 


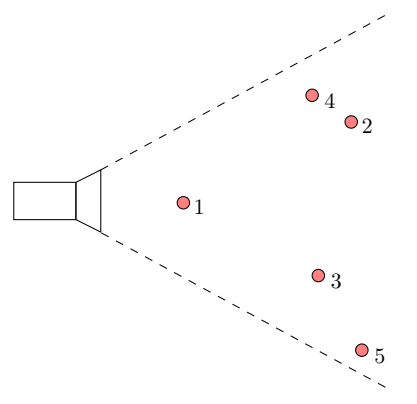

(a)

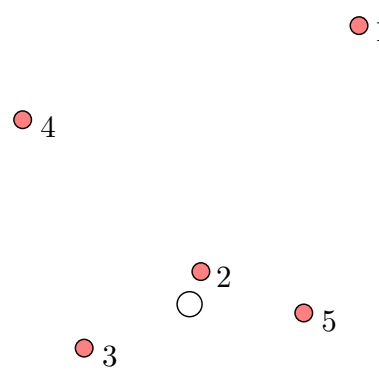

(b) $\bigcirc_{1}$

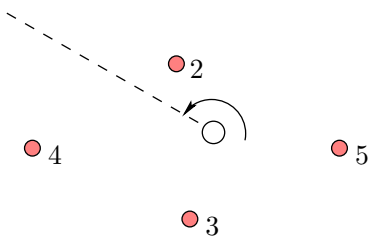

(c)

Figure 16: Three kinds of compound relational sensors: (a) The linear sensor observes that the landmarks are ordered from left to right as $(4,2,1,3,5)$. (b) This sensor sorts the landmarks closest to farthest, resulting in the observation $(2,3,5,4,1)$. (c) The cyclic sensor sweeps counterclockwise and yields the cyclic ordering $(1,2,4,3,5)$

Model 21 (Primitive Relational Sensor)

This sensor indicates whether the relation $R_{x}$ is satisfied for two bodies $B_{i}$ and $B_{j}$ that are in the detection region:

$$
h(x)= \begin{cases}1 & \text { if } R_{x}(i, j) \\ 0 & \text { otherwise }\end{cases}
$$

Numerous instantiations of Model 21 can be used in combination to obtain compound relational sensors. The idea is to make a sensor that produces a vector of binary observations, one from each primitive. The resulting observation can be considered as a graph $G_{x}$ for which the vertices are the set of bodies and a directed edge exists if and only if $R_{x}(i, j)$. As the state changes, the edges in $G_{x}$ may change.

An important compound relational sensor will now be defined.

Model 22 (Linear Permutation Sensor)

Suppose there is a finite set of static point bodies in the plane that are considered as completely distinguishable landmarks. Consider a relation $\preceq_{l}$, for which $a \preceq_{l} b$ means that $a$ appears to be to the left of $b$ when viewed from the sensor position $\left(q_{x}, q_{y}\right)$. If these are in the field of view of a camera, we should be able to determine the value of the relation for any pair of points. See Figure 16(a). The binary observations that determine $\preceq_{l}$ can be combined to yield a single observation that is a linear ordering of the landmarks. In the example, the observation would be $y=(4,2,1,3,5)$. If the landmarks were capable of moving, then any permutation might be possible, and $Y$ would be the set of all 5 ! permutations.

It is tempting to make primitive relations that have more than two outputs, especially if the bodies appear in some degenerate positions. For example, the sensor might not be able to determine whether $a$ is to the left or right of $b$ because they are perfectly aligned in the sensor view. Such cases can be handled by defining multiple relations. For example, one primitive could be $\preceq_{l}$, and a new one $\preceq_{a}$ could indicate whether they are aligned.

Model 23 (Distance Permutation Sensor)

Figure 16(b) shows how to obtain an alternative permutation based on sorting the bodies from 
nearest to farthest. In practice, imagine that each landmark has a radio transmitter. A sensor that measures the signal strengths could in principle sort them according to strength, and hence distance. This would work only under idealized conditions. In practice, it might be preferable to allow the sensor to report that two landmarks are of approximately equal distance away, when it is unable to reliably decide which is further.

For some problems, two-argument relations are insufficient. For example, we might want a primitive observation that tells whether point $p_{k}$ is to the left or right of a ray that starts at point $p_{i}$ and pierces point $p_{j}$. This relation involves triples of points, and can be expressed as $R_{x}(i, j, k)$. This relation can be used to define the next model.

Model 24 (Cyclic Permutation Sensor)

We extend Model 22 (linear permutation) to a sensor that performs a $360^{\circ}$ sweep. In this case, the notion of "left of" is not well defined because of the cyclic ordering. However, for a set of three points, $a, b$, and $c$, we can determine whether the cyclic permutation is $(a, b, c)$ or $(a, c, b)$ (note that others are equivalent, such as $(b, c, a)=(a, b, c))$. When the primitive observations are combined, the compound sensor in this case yields a cyclic permutation of the landmarks, as shown in Figure $16(\mathrm{c})$.

If the bodies are only partially distinguishable, then many interesting relational sensor variants arise.

\subsubsection{Gap Sensors}

This next family of sensor models is closely related to the previous three families. The idea is to report information obtained along the boundary of $V(q)$, which is denoted as $\partial V(q)$. For most 2D cases, $\partial V(q)$ is a closed curve. To motivate this model, recall Model 12 (omnidirectional depth), Figure 9(d), and Figure 10. The data from the omnidirectional depth sensor are depicted again in Figure 17(a), but this time discontinuities or gaps in the depth measurements are shown. When sweeping counter-clockwise, imagine a sensor that reports: A wall, then a gap $g_{1}$, then a wall, then a gap $g_{2}$, then a wall, and so on. The alternation between an obstacle or body and a gap in the distance measurements is the information provided by a gap sensor. In general, a gap sensor observation is a sequence, for example $\left(B_{2}, g_{1}, B_{3}, g_{2}, B_{1}\right)$, which alternates between bodies and gaps. Examples will be given in which this sequence is linear or cyclic. For the mobile robot models in Section 3.2.4, the complement of $E$ can be treated as a static body, so that the observation alternates between gaps and the environment boundary.

Model 25 (Simple Gap Sensor)

This sensor has already been described using Figure 17(a). Suppose that a robot carries a sensor with an omnidirectional field of view and is placed into a nondegenerate environment $E$ that bounded by a simple polygon and contains no interior obstacles. Treating the complement of $E$ as a special body, say $B_{0}$, the gap sensor for Figure 17(a) observes

$$
y=\left(B_{0}, g_{1}, B_{0}, g_{2}, B_{0}, g_{3}, B_{0}, g_{4}, B_{0}, g_{5}\right),
$$

which is interpreted as a cyclic sequence. Since it is impossible to have two consecutive gaps, the $B_{0}$ components contain no information, and (18) can be simplified to $y=\left(g_{1}, g_{2}, g_{3}, g_{4}, g_{5}\right)$. Once again, this observation is cyclic; for example, $y=\left(g_{3}, g_{4}, g_{5}, g_{1}, g_{2}\right)$ is equivalent. 


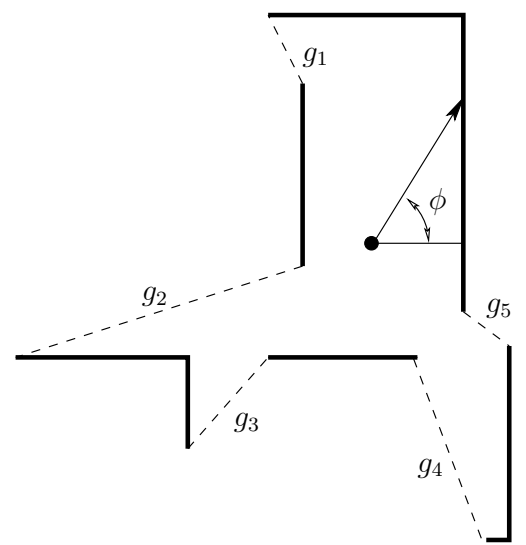

(a)

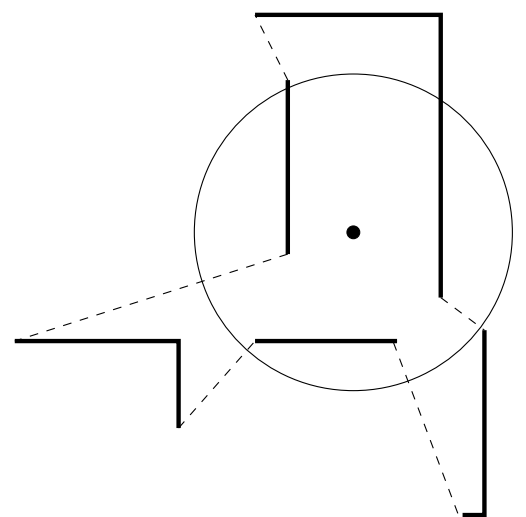

(b)

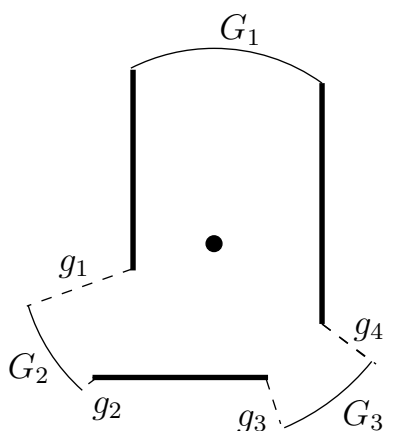

(c)

Figure 17: Gap sensor models: (a) Five discontinuities in depth are observed. (b) A limited range is considered. (c) Two kinds of gaps are obtained for limited range.

Model 26 (Depth-Limited Gap Sensor ${ }^{2}$ )

In reality, most sensors have limited range. Suppose that for an omnidirectional sensor, nothing can be sensed beyond some fixed distance, as shown in Figure 17(b). The resulting data from a depth sensor would appear as in Figure 17(c). There are two kinds of gaps: one from a discontinuity in depth and the other from a range of angles where the depth cannot be measured because the boundary is too far away. Let the discontinuity gaps be labeled $g_{i}$, as before, and the new gaps be labeled $G_{i}$. The observation for the example in Figure $17(\mathrm{c})$ is $y=\left(B_{0}, G_{1}, B_{0}, g_{1}, G_{2}, g_{2}, B_{0}, g_{3}, G_{3}, g_{4}\right)$, which again is a cyclic sequence. In contrast to Model 25 (simple gap), the appearances of $B_{0}$ cannot be deleted without losing information.

\section{Model 27 (Multibody Gap Sensor)}

In the models so far, only one body, $B_{0}$, was considered. Now suppose there are multiple bodies, as shown in Figure 18(a). The sensor sweeps from right to left, and is not omnidirectional. In this case, the observation is a linear sequence,

$$
y=\left(G_{1}, g_{1}, B_{4}, g_{2}, B_{5}, g_{3}, B_{4}, g_{4}, G_{2}, g_{5}, B_{3}, g_{6}, B_{2}, g_{7}, B_{1}\right) .
$$

For Model 27, it was assumed that the bodies are completely distinguishable. As in Model 20, it is once again possible assign labels to be bodies. In this case, Model 27 could be extended so that the observation yields a sequence of gaps and labels, as opposed to gaps and bodies.

Following along these lines, the next model simply counts the number of bodies between gaps. It is based on a model called the combinatorial visibility vector in [19].

Model 28 (Landmark Counter)

Let $E$ be a bounded environment with no interior holes. Let the bodies be a finite set of points that are static and distributed at distinct locations along the boundary of $E$. All bodies are assigned

\footnotetext{
${ }^{2}$ This model is based on the one introduced in [42].
} 


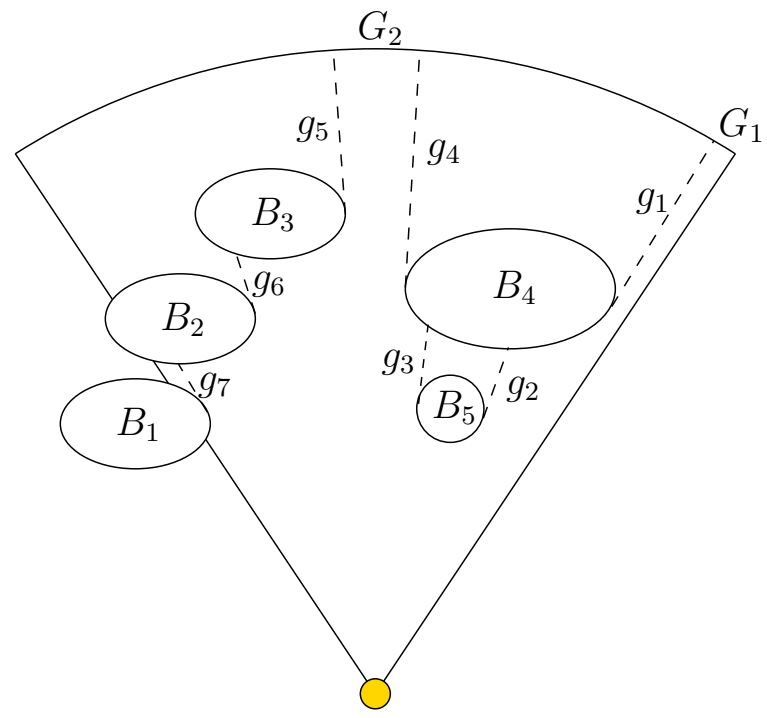

(a)

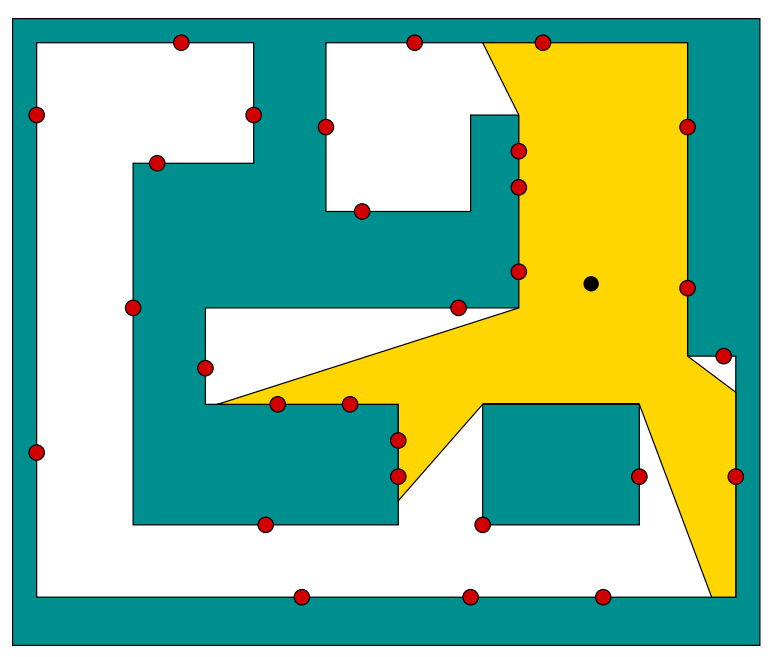

(b)

Figure 18: (a) A gap sensor among multiple bodies. (b) A sensor that counts landmarks between gaps.

a common label, such as "feature", meaning that they are completely indistinguishable. When in the interior of $E$, the sensor observation is a cyclic sequence of integers, corresponding to the number of bodies between each pair of gaps. The observation for the example in Figure 18(b) is $y=(3,3,4,0,1)$.

The model can be adapted in several ways: 1) a linear sequence could be obtained by placing the sensor on the boundary, or by observing the starting point of the omnidirectional sweep, 2) any level of partial or full distinguishability of bodies could be allowed, 3) the bodies could be placed in the interior, and 4) the bodies could be capable of motion.

\subsection{The Sensor Lattice}

After seeing so many sensor models, you might already have asked, what would it mean for one sensor to be more powerful than another? It turns out that there is a simple, clear way to determine this in terms of preimages.

For all of the discussion in this section, assume that the state space $X$ is predetermined and fixed. Let $h_{1}: X \rightarrow Y_{1}$ and $h_{2}: X \rightarrow Y_{2}$ be any two sensor models (recall the great variety from Section 3.2). A partition $\Pi_{1}$ is called a refinement of $\Pi_{2}$ if every set in $\Pi_{1}$ is a subset of some set in $\Pi_{2}$. We say that $h_{1}$ dominates $h_{2}$ if and only if $\Pi\left(h_{1}\right)$ is a refinement of $\Pi\left(h_{2}\right)$. This is denoted as $h_{1} \succeq h_{2}$.

For some state $x \in X$, imagine receiving $y_{1}=h_{1}(x)$ and $y_{2}=h_{2}(x)$. If $h_{1} \succeq h_{2}$, then $h_{1}^{-1}\left(y_{1}\right) \subseteq h_{2}^{-1}\left(y_{2}\right) \subseteq X$. This clearly means that $h_{1}$ provides at least as much information about $x$ as $h_{2}$ does. Furthermore, using $y_{1}$, we could infer what observation $y_{2}$ would be produced by $h_{2}$. Why? Since $\Pi\left(h_{1}\right)$ is a refinement of $\Pi\left(h_{2}\right)$, then every $x \in h_{1}^{-1}\left(y_{1}\right)$ must produce the same observation $y_{2}=h_{2}(x)$. This implies that there exists a function $g: Y_{1} \rightarrow Y_{2}$ such that $h_{2}(x)=g\left(h_{1}(x)\right)$, written as $h_{2}=g \circ h_{1}$. Here is a diagram of the functions: 


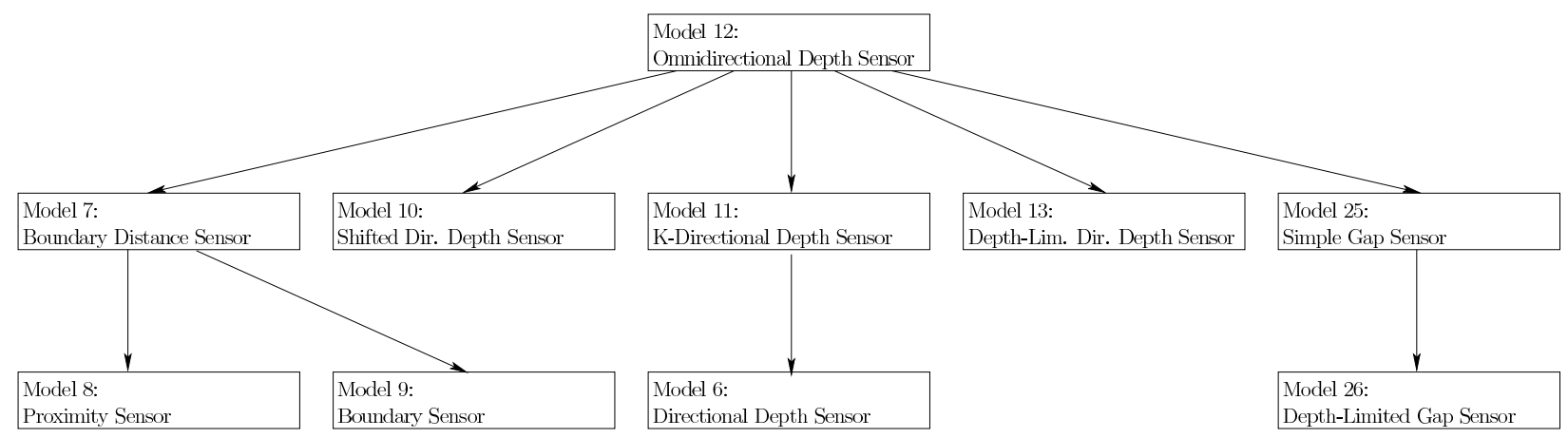

Figure 19: Several models from Section 3.2 are related using the idea of dominance, based on refinements of the partitions they induce over $X$. Models higher in the tree induce finer partitions. A lower sensor model can be "simulated" by any model along the path from the root of the tree to itself.

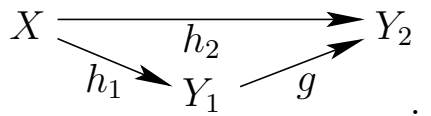

The existence of $g$ implies that $h_{1}$ 's observations can be used to "simulate" $h_{2}$, without needing additional information about the state. One important point, however, is that it might be computationally impractical or infeasible to compute $g$ in practice. The decidability and complexity of computing $g$ lead to interesting open research questions for various sensing models.

Using the dominance relation $\succeq$, we can naturally compare many of the sensors in Section 3.2. Note that $\succeq$ is a partial ordering; most sensor pairs are incomparable. Figure 19 shows how some sensors are related. The most powerful sensor of Section 3.2.4 is the omnidirectional depth sensor because it induced the finest partition of $X$. We can use it to simulate all other sensors in that section. For the directional sensors, it is assumed that the directions are properly aligned. Since gaps are just discontinuities in the depth function, the depth sensors can even be used to simulate gap sensors, such as Models 25 (simple gap) and 26 (depth-limited gap). Note that these relationships hold regardless of the particular collection $\mathcal{E}$ of possible environments. It does not matter whether the environment is given or is open to some infinite collection of possibilities.

Other sensors could be added to Figure 19. For example, the Model 1 (dummy), is dominated by all of these sensors. Furthermore, Model 2 (identity) dominates all of these. The same is true of Model 3 (bijective), since both induce the same partition of $X$.

What happens as we include more and more sensors, and continue to extend the diagram in Figure 19? It is truly remarkable that all possible sensors of the form $h: X \rightarrow Y$ over a fixed state space $X$ can be related in a clear way, and the tree extends into a lattice.

Note that $Y$ is not fixed, meaning we could take any set $Y$ and define any mapping $h: X \rightarrow Y$. Consider defining an equivalence relation $\sim$ on this enormous collection of sensors: We say that $h_{1} \sim h_{2}$ if and only if $\Pi\left(h_{1}\right)=\Pi\left(h_{2}\right)$. For example, Models 2 (identity) and 3 (bijective) are equivalent because the both induce the same partitions of $X$ (all preimages are singletons). More precisely, Model 3 is a family of sensors, which includes Model 2; however, the entire family is equivalent.

If we no longer pay attention to the particular $h$ and $Y$, but only consider the induced partition of $X$, then we imagine that a sensor is a partition of $X$. Continuing in this way, the set of all possible sensors is the set of all partitions of $X$.

The relationship between sensors in terms of dominance then leads to the well-known idea of a partition lattice, depicted in Figure 20 for the set $X=\{1,2,3,4\}$. Recall that a lattice is a set 


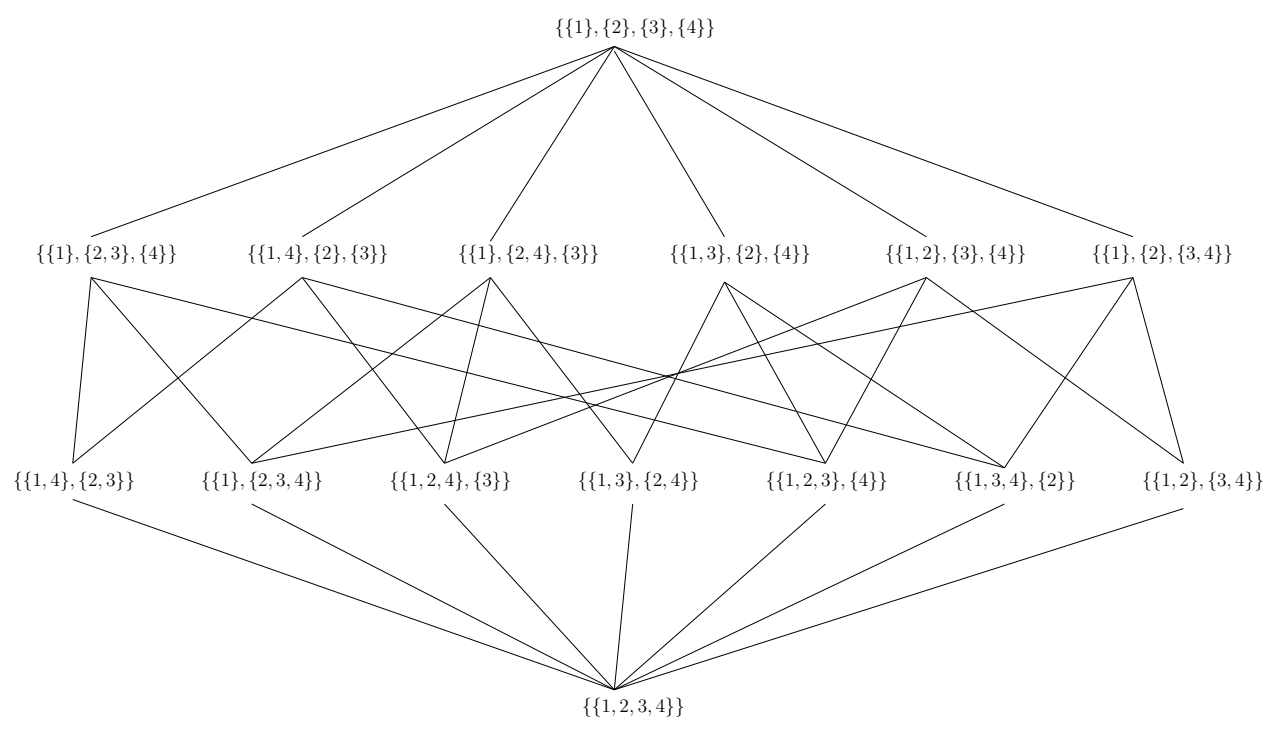

Figure 20: The partition lattice for a four-element set. The best and worst sensors are at the top and bottom, respectively.

together with a partial order relation $\succeq$ for which every pair of elements has a least upper bound (lub) and a greatest lower bound (glb). Starting with any set, the set of all partitions forms a lattice. The relation $\succeq$ is defined using refinements of partitions: $\pi_{1} \succeq \pi_{2}$ if and only if $\pi_{1}$ is a refinement of $\pi_{2}$.

Now observe that for any state space $X$, all possible sensors fit nicely into the partition lattice of $X$. Furthermore, $\succeq$ indicates precisely when one sensor dominates another. The tree depicted in Figure 19 is embedded in this lattice. The partition corresponding to Model 3 (bijective), is at the top of the lattice because it is the finest partition possible. Model 1 (dummy) is at the bottom of the lattice because it is the coarsest partition possible.

An important property of a lattice is that every pair of elements has a unique glb and a unique lub. These have interesting interpretations in the sensor lattice. Suppose that for two partitions, $\Pi\left(h_{1}\right)$ and $\Pi\left(h_{2}\right)$, neither is a refinement of the other. Let $\Pi\left(h_{3}\right)$ and $\Pi\left(h_{4}\right)$ be the glb and lub, respectively, of $h_{1}$ and $h_{2}$. The glb $\Pi\left(h_{3}\right)$ is the partition obtained by "overlaying" the partitions $\Pi\left(h_{1}\right)$ and $\Pi\left(h_{2}\right)$. Take any state $x \in X$. Let $y_{1}, \ldots, y_{4}$, be the observations obtained by applying $h_{1}, \ldots, h_{4}$, respectively. An element of $\Pi\left(h_{3}\right)$ is obtained by intersecting preimages, $h_{1}^{-1}\left(y_{1}\right) \cap h_{2}^{-1}\left(y_{2}\right)$. There is a straightforward way to construct some representative $h_{3}$ from $h_{1}$ and $h_{2}$. Let $Y_{3}=Y_{1} \times Y_{2}$ and $h_{3}: X \rightarrow Y_{3}$ be defined as $y_{3}=\left(y_{1}, y_{2}\right)=\left(h_{1}(x), h_{2}(x)\right)$. This means that both $h_{1}$ and $h_{2}$ are combined to produce a single sensor. The partition $\Pi\left(h_{3}\right)$ is just the common refinement.

The lub, $\Pi\left(h_{4}\right)$, is the opposite of $\Pi\left(h_{3}\right)$ in some sense. The partition $\Pi\left(h_{4}\right)$ is as coarse as it needs to be so that every element contains the complete preimages of $h_{1}$ and $h_{2}$. Again starting from any $x \in X, \Pi\left(h_{4}\right)$ is the finest partition for which $h^{-1}\left(y_{1}\right) \cup h^{-1}\left(y_{2}\right) \subseteq h^{-1}\left(y_{3}\right)$.

One way to "visualize" these relationships is to imagine the case in which $X=Y=\mathbb{R}^{3}$ and restrict the set of all sensor mappings to be only linear ones, $y=C x$, as given in Model 4 (linear). If $C$ has rank 2 , then the preimages $h^{-1}(y)$ are lines in $\mathbb{R}^{3}$. Consider two linear sensors, with matrices $C_{1}$ and $C_{2}$ having rank 2 . The glb produces preimages that are the intersection of two lines. The lines must always intersect because both preimages are observations of same state $x \in \mathbb{R}^{3}$. If the combined 3 by 6 matrix, $\left[C_{1} C_{2}\right]$, has rank three, then all preimages will be points, and the glb is a bijective sensor. The preimages for the lub in this case is the set of all planes in $\mathbb{R}^{3}$. Each plane 
is obtained by taking the union of the preimages, which forms a pair of intersecting lines.

\subsection{Additional Complications}

Up until now, all sensor models have been idealized because the observation is immediately and completely determined by applying $y=h(x)$. Although preimages $h^{-1}(y)$ reveal important information about sensing problems, additional concerns have so far been neglected. This section handles three independent issues: 1) the sensor observation might not be predictable, even if the state is known, 2) the observation might depend on state and time, and 3) the observation might depend on one or more previous states, rather than being instantaneous.

\subsubsection{Nondeterministic Disturbance}

Suppose that a sensor provides an observation instantaneously, but there is uncertainty about which observation will occur at state $x$. We consider two general models to address this: Nondeterministic and probabilistic. First, the nondeterministic model will be defined, which might alternatively be called possibilistic. In this case, the sensor mapping specifies a set of possible observations that could be obtained from some state $x \in X$.

Let an observation space $Y$ be defined as before and let $X$ be any state space. A nondeterministic sensor mapping is defined as

$$
h: X \rightarrow \operatorname{pow}(Y)
$$

in which pow $(Y)$ is the power set of $Y$, to yield any possible subset. For a state $x \in X$, the mapping $h$ yields a set $h(x) \subseteq Y$. An alternative to (20) is to define a nature action that generates a disturbances and construct a function that maps states and nature actions into observations; see Section 11.1 of [37].

Before giving examples of (20), consider the effect on preimages. A reasonable definition of a preimage for a nondeterministic sensor mapping $h$ is

$$
h^{-1}(y)=\{x \in X \mid y \in h(x)\} .
$$

(Note that this is not a preimage in the usual sense for functions because $y$ is not an element of pow $(Y)$.) If $h(x)$ is a singleton subset for all $x \in X$, then (21) reduces to the original preimage (2). As $h(x)$ grows in size, the preimages become larger. Rather than a partition of $X$, a cover of $X$ is obtained. This means that the union of all $h(x)$ is equal to $X$, but the preimages are not necessarily disjoint.

Model 29 (One-Dimensional Position Sensor)

Let $X=Y=\mathbb{R}$. Imagine a sensor that measures the position along $X$. Let $\epsilon>0$ be a bound in the maximum amount of measurement error. The nondeterministic sensor mapping is

$$
h(x)=\{y \in Y|| x-y \mid \leq \epsilon\} .
$$

For example, $h(2)=[2-\epsilon, 2+\epsilon]$. The actual observation produced by the sensor may be any value $y \in[2-\epsilon, 2+\epsilon]$.

The preimage of an observation $y$ is

$$
h^{-1}(y)=\{x \in X|| x-y \mid \leq \epsilon\} .
$$

For example, $h^{-1}(5)=[5-\epsilon, 5+\epsilon]$. Clearly, the preimages of $h$ yield a cover of $X=\mathbb{R}$. 
Nondeterministic versions of the sensors in Section 3.2 can be easily constructed. A couple of examples are given here.

Model 30 (K-Dimensional Position Sensor)

Model 29 can be easily extended to $k$ dimensions, usually with $k=2$ or $k=3$. Reusing $\epsilon>0$, the sensor mapping could be

$$
h(x)=\{y \in Y \mid\|x-y\| \leq \epsilon\},
$$

in which $\|\cdot\|$ is the Euclidean norm. In this case, $h(x)$ is a disc of radius $\epsilon$, as are the preimages of $h$.

Model 31 (Faulty Detectors)

Consider modifying a static binary sensor given by (11). The sensor might produce a false positive by yielding $h(p, E)=1$ even though $p \notin V$. In this case, the preimage would be $h^{-1}(1)=X$. If the sensor could also produce a false negative by yielding $h(p, E)=0$ when $p \in V$, then $h^{-1}(0)=X$. These two preimages together cover $X$ twice, and we clearly see that the sensor is absolutely worthless under this model: We can infer nothing about the state from the observation if false negatives and false positives are permitted. In practice, a sensor that commits such errors might nevertheless be useful, but probabilistic modeling is then needed (how likely is it to make a mistake?); this is the subject of Section 3.4.2.

The notion of dominance from Section 3.3 can be extended to the nondeterministic sense. Once again, we must determine whether one sensor can "simulate" the other. We say that $h_{1}$ dominates $h_{2}$ if there exists a mapping $g: Y_{1} \rightarrow \operatorname{pow}\left(Y_{2}\right)$ such that for all $y_{1} \in h_{1}(x), g\left(y_{1}\right) \subseteq h_{2}(x)$. Several alternative domination definitions are possible in this context.

Model 32 (Inaccurate Directional Depth)

Recall Model 6 (directional depth). For any $\epsilon \geq 0$, we can define a mapping

$$
h_{\epsilon}(p, \theta, E)=\{y \in[0, \infty)||\|p-b(x)-y\| \mid \leq \epsilon\} .
$$

Note the similarity to (22). For this model, $h_{\epsilon}$ dominates $h_{\epsilon^{\prime}}$ if and only if $\epsilon \leq \epsilon^{\prime}$.

\subsubsection{Probabilistic Disturbance}

Perhaps we have been observing a sensor over many trials and are able to better characterize the disturbances. Rather than simply talking about the set of possible observations, we could statistically learn a probability density over them. Start with (20), in which $h(x)$ yields a set of possible observations in $Y$. The models in this section place a probability density over $h(x)$. A convenient way to express this is

$$
p(y \mid x),
$$

which is a probability density function over $Y$, but conditioned on the particular state, $x \in X$. Unfortunately, this representation hides the underlying sensor mapping. Using $h$ that we can declare $p(y \mid x)=0$ for all $y \notin h(x)$, which is powerful information that is not reflected in $(26)$. Furthermore, all of the important preimage and cover information is obscured. It is therefore critical when using probabilistic models to recall and utilize the underlying structure of the sensor mapping $h$.

Some probabilistic sensor models will now be defined. 
Model 33 (Probabilistic 1D Position Sensor)

We first make a probabilistic variant of Model 29 (one-dimensional position). Assume the error density is Gaussian with zero mean and variance $\sigma^{2}$. The probability density function is

$$
p(y \mid x)=\frac{1}{\sigma \sqrt{2 \pi}} e^{-\frac{(x-y)^{2}}{2 \sigma^{2}}} .
$$

This function is maximized when $x=y$, which corresponds to the case of no disturbance.

Model 34 (Probabilistic General Position Sensor)

Now consider a probabilistic variant of Model 30 (k-dimensional position). Assume the error density is Gaussian with zero mean and $\Sigma$ as a $k \times k$ covariance matrix. The density function is

$$
p(y \mid x)=\frac{1}{|\Sigma|^{1 / 2}(2 \pi)^{k / 2}} e^{(y-x)^{T} \Sigma^{-1}(y-x)} .
$$

Model 35 (Probabilistic Detectors)

Revisiting Model 31 (faculty detectors), simply attach probabilities to false positives and false negatives. For a false positive, we define $p(y=1 \mid p \notin V)$. The condition $p \notin V$ could be replaced with a more precise location for $p$, to allow conditional probabilities that vary because of the state. Note that the "correct" positive is obtained by subtracting the above probability from one. Likewise, a false negative is defined by $p(y=0 \mid p \in V)$.

Model 36 (Probabilistic Directional Depth)

A generalization of Model 32 (inaccurate directional depth), again assuming a zero-mean Gaussian density for disturbances, is

$$
p(y \mid p, \theta, E)=\frac{1}{\sigma \sqrt{2 \pi}} e^{-\frac{(y-\|p-b(x)\|)^{2}}{2 \sigma^{2}}} .
$$

\subsubsection{Sensors Over State-Time Space}

Recall from Section 3.1.3, that once time is introduced, the state-time space $Z=X \times T$ is used to fully describe any situation. In this case, the sensor should logically be defined over $Z$, rather than $X$. This means that a combination of state $x \in X$ and time $t \in T$ produces an observation $y$. Thus, the basic sensor mapping (1) is replaced by

$$
h: Z \rightarrow Y \text {, }
$$

for which we write $y=h(z)$, or equivalently, $y=h(x, t)$.

Here is a simple example.

Model 37 (Perfect Clock)

This sensor simply reports the current time:

$$
y=h(z)=h(x, t)=t .
$$


The basic sensing examples from Section 3.2 .3 can be easily extended by replacing $X$ with $Z$. They could, for example, report time in addition to their existing observation. Here is an example that extends Model 14 (static binary detector).

Model 38 (Detector with Time Stamp)

Use the same model for $V, E$, and $p$ as in Model 14. Let $Y=\{0,1\} \times T$. The sensor mapping $h: Z \times Y$ is

$$
h(p, E, t)= \begin{cases}(1, t) & \text { if } p \in V \text { at time } t \\ (0, t) & \text { otherwise. }\end{cases}
$$

All of the concepts from Section 3.2.2 extend naturally from $X$ to $Z$. A preimage under the sensing model in (30) is

$$
h^{-1}(y)=\{(x, t) \in Z \mid y=h(x, t)\},
$$

Now consider partitions $\Pi(h)$ over $Z$. A weak sensor may partition $Z$ into large chunks of state-time space. Following Section 3.3, a sensor $h_{1}$ dominates another $h_{2}$ if and only if its partition $\Pi\left(h_{1}\right)$ of $Z$ is a refinement of $\Pi\left(h_{2}\right)$. In the same way as for $X$, a partition lattice over $Z$ is obtained.

The concepts from Sections 3.4.1 and 3.4.1 can be adapted here to yield nondeterministic and probabilistic sensor models. This results in $h: Z \rightarrow \operatorname{pow}(Y)$ for the nondeterministic case and $p(y \mid z)$ for the probabilistic case.

\subsubsection{History-Based Sensors}

As a natural transition to the temporal filters of Section 4, we consider one final extension to the sensor models. It might be the case that the sensor output depends on a history of previous states. The most common examples in practice are odometers, such as the wheel encoder in Figure 4(e). They accumulate changes over time and report the aggregate amount, such as total distance traveled. The relationship to Section 4 is that the sensors here could be realized by employing a filter that uses information from instantaneous sensors (such as $h: X \rightarrow Y$ ). In other words, a history-based sensor usually contains a built-in filter. This should become clearer after reading Section 4.

Let a state trajectory up to time $t$ be denoted as $\tilde{x}:[0, t] \rightarrow X$. The set of all possible trajectories for any possible $t \in T$ is called the trajectory space and is denoted by $\tilde{X}$. For a history-based sensor, the sensor mapping is

$$
h: \tilde{X} \rightarrow Y \text {. }
$$

In this case, a given state trajectory $\tilde{x}$ produces an observation $y=h(\tilde{x})$.

Once again, the notions of preimages, partitions, and the sensor lattice naturally extend. In this case, $X$ is simply replaced by $\tilde{X}$. For example,

$$
h^{-1}(y)=\{\tilde{x} \in \tilde{X} \mid y=h(\tilde{x})\}
$$

yields the set of possible trajectories in $\tilde{X}$ that produce the same $y$. The preimages induce a partition of $\tilde{X}$, and all history-based sensors can be arranged into a sensor lattice over $\tilde{X}$.

Some examples of history-based sensors follow. 
Model 39 (Linear Odometer)

Suppose we would like to measure how far a robot has traveled. Let $\left(v_{1}, v_{2}\right)$ represent the instantaneous velocity of a planar robot. A history-based sensor could integrate the magnitude of velocity obtain the distance:

$$
y=\theta_{0}+\int_{0}^{t} \sqrt{v_{1}^{2}+v_{2}^{2}} d s .
$$

This model implies that $v_{1}$ and $v_{2}$ are components of state (as is common in classical mechanics and modern control theory). For a robot moving in a given planar environment, the state could be represented as $x=\left(p_{1}, p_{2}, \theta, v_{1}, v_{2}\right)$.

Model 40 (Angular Odometer)

Recall the wheel encoder of Figure 4(e). An idealized model of this sensor can be made by considering the limit after making smaller and smaller holes along the disc. This results in a perfect angular odometer, which is modeled as

$$
y=\theta_{0}+\int_{0}^{t} \dot{\theta}(s) d s
$$

in which $y$ measures the net orientation change from some starting orientation. What would the sensor report if $|\dot{\theta}(s)|$ is integrated instead?

In practice, sensors cannot actually produce instantaneous observations. Using a history-based sensor, the delay can be explicitly modeled:

Model 41 (Delayed Measurement)

Suppose a sensor measures the state perfectly, but it takes one unit of time to output the result. This can be modeled as

$$
y= \begin{cases}\tilde{x}(t-1) & \text { if } t \geq 1 \\ \# & \text { otherwise }\end{cases}
$$

in which \# means that the state cannot yet be determined. A delayed version of any sensor of the form $h: X \rightarrow Y$ or $h: Z \rightarrow Y$ can be made in this way.

Model 42 (Discrete-Time Odometer)

Without referring directly to velocities, a history-based sensor can be constructed that estimates the distance traveled by comparing positions reported at various times. Consider some $\Delta t>0$, corresponding to a fixed time increment. Let $\tilde{p}(t)$ denote the robot position in $\mathbb{R}^{2}$ at time $t$; this can be determined from $\tilde{x}(t)$.

The sensor mapping is

$$
h(\tilde{x})=\sum_{i=1}^{\lceil t / \Delta t\rceil}\|\tilde{p}(i \Delta t)-\tilde{p}((i-1) \Delta t)\| .
$$

For a state trajectory $\tilde{x}:[0, t] \rightarrow X$, the total distance traveled is estimated. The quality of the estimate depends on how small $\Delta t$ is selected. This sensor is essentially constructed as a temporal filter, which will be covered in Section 4.2. 


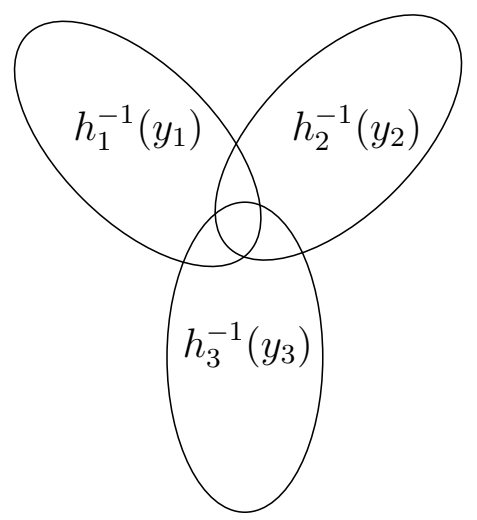

Figure 21: Triangulation is generally viewed as an intersection of preimages.

\section{$4 \quad$ Filtering}

A filter combines information from multiple observations to efficiently keep track of state properties that are needed for inference tasks. The observations may come from different sensors, which either appear in the same location or are distributed around the same environment. In these cases, a spatial filter sews the information together to make a coherent view. Alternatively, the observations may arrive sequentially over time from the same sensor. This leads to a temporal or causal filter, which incrementally updates its internal information for each new observation. A filter may even combine both spatial and causal elements. In the most general settings, we must resolve observations taken at different times from various sensors distributed around the environment.

\subsection{Spatial Filters}

Here we suppose that some sensors have been distributed around the environment and each has produced an observation. How is this information interpreted? The answer is mostly provided by analyzing preimages, which were introduced in Section 3.2.2.

\subsubsection{A general triangulation principle}

Suppose that several instantaneous sensors produce their observations at the same time. Techniques have been widely used for hundreds of years for combining information from these to obtain important quantities such as distance, longitude, and latitude. Most of this section is a generalization of the ancient idea of triangulation, in which observing the angles between pairs of distant features has been used to infer location. We now develop this idea in a general way using concepts of Section 3.

Consider any $n$ sensor mappings $h_{i}: X \rightarrow Y_{i}$ for $i$ from 1 to $n$. If each produces an observation $y_{i} \in Y_{i}$ at some common instant, then what are the possible states? The triangulation ${ }^{3}$ of the observations is denoted by $\Delta\left(y_{1}, \ldots, y_{n}\right)$. It is determined by intersecting the preimages (2) of each sensor to obtain (see Figure 21)

$$
\Delta\left(y_{1}, \ldots, y_{n}\right)=h_{1}^{-1}\left(y_{1}\right) \cap h_{2}^{-1}\left(y_{2}\right) \cap \cdots \cap h_{n}^{-1}\left(y_{n}\right),
$$

which is a subset of $X$. It is the set of all states that could possibly have produced the $n$ observations simultaneously.

\footnotetext{
${ }^{3}$ This is completely different from triangulations in computational geometry, topology, or meshing.
} 


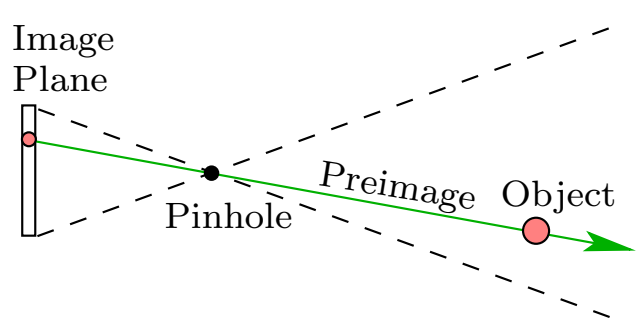

a.

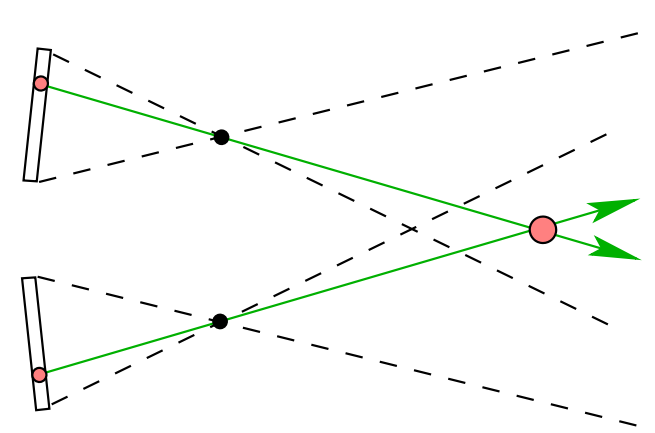

b.

Figure 22: a) Using the pinhole camera model, the preimage of a point object is an infinite ray that that connects from the pinhole to the object. b) From matching the same object in two camera images, its location can be determined by intersecting the two preimage rays.

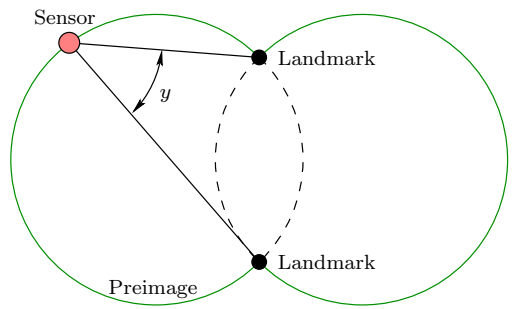

a.

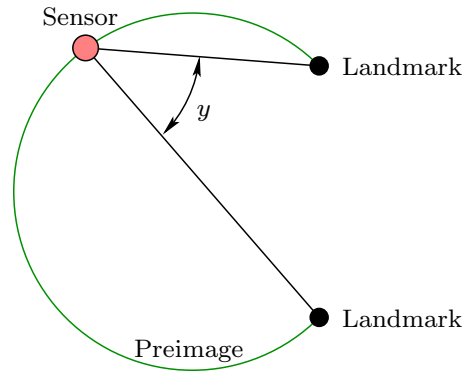

b.

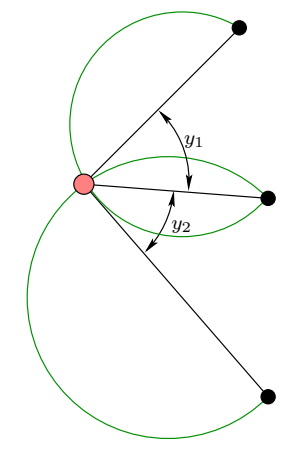

c.

Figure 23: The ancient triangulation technique uses observations of the angle between pairs of landmarks. (a) Holding the angle between two landmarks fixed, the preimage is a portion of two circles. (b) If it is known which landmark is to the left, then half of the original preimage is eliminated to obtain a circular arc. (c) With three landmarks, with known left-to-right order, the circular arcs are intersected to determine the sensor location.

Here are some important, classical examples of triangulation.

Filter 1 (Stereopsis)

Figure 22(a) shows a small object appearing in an image under the pinhole camera model. This can be imagined as an object in $X=\mathbb{R}^{3}$ with a $2 \mathrm{D}$ image, or an object in $X=\mathbb{R}^{2}$ with a onedimensional image. The sensor mapping $h: X \rightarrow Y$ is the standard perspective projection model, in which $y \in Y$ represents the location of the object in the image. The preimage $h^{-1}(y)$ is a ray that extends outward from the pinhole and through the object in $\mathbb{R}^{3}$. Figure 22(b) illustrates the principle of stereopsis, commonly used to locate objects in human vision and computer vision systems. In this case, the preimages are intersected to reveal the precise object location. This assumes that the positions and orientations of the cameras are known.

Filter 2 (Ancient Triangulation)

Figure 23 shows classical triangulation, which is a technique used for thousands of years by an- 


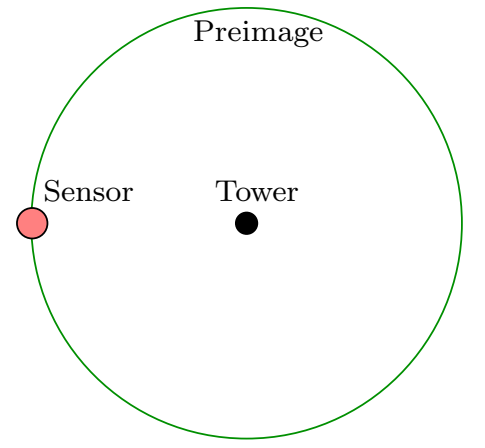

a.

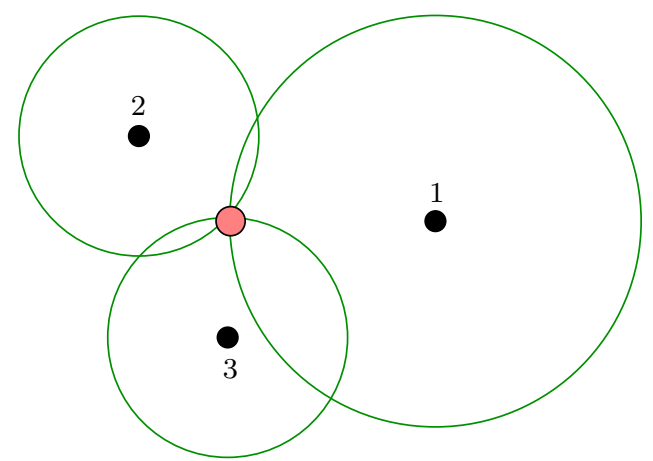

b.

Figure 24: The principle of trilateration enables the sensor location to be determined from observations of distances to known towers. a) From a single tower, the sensor could be anywhere along a circle. b) Using three towers, the position is uniquely determined.

cient Greeks, Egyptians, and Chinese. The sensor mapping provides the angle between a pair of landmarks, as observed from the sensor location. To understand the preimage, depicted in Figure 23(a), imagine moving around in the plane while holding the angle between two landmarks fixed. What curve do you trace? It turns out to be a circular arc; however, there are two arcs depending on whether you are "in front of" or "behind" the landmarks. The example shown is for angle observations $y<\pi / 2$. If $y=\pi / 2$, then the two arcs fuse into a single circle. If $y>\pi / 2$, then the preimages appears as the dashed lines in Figure 23(a). If there is not front/behind ambiguity, then one arc can be eliminated, as depicted in Figure 23(b). If there are three landmarks, then two angles are obtained and the preimage arcs are intersected to obtain the precise sensor location, shown in Figure 23(c).

\section{Filter 3 (Trilateration)}

Figure 24 shows the principle of trilateration. Rather than observing the angle between landmarks, imagine that a sensor observes the distance to a landmark. If both the landmark and sensor have synchronized clocks, then a virtual sensor can be constructed that estimates the distance based on the time of arrival (TOA) of the signal. This assumes a known propagation speed for sound waves or radio signals. Suppose that $X=\mathbb{R}^{2}$ and the landmark location is known. The sensor mapping $h: X \rightarrow Y$ yields a distance $y=h(x) \in(0, \infty)$. The preimages $h^{-1}(y)$ are circles of radius $y$, centered at the landmark.

Now consider determining the precise sensor location. If there are two landmarks, the intersection of preimages yields a pair of points because two circles intersect at two points in general. If there are three landmarks, then three circles are intersected to obtain the precise sensor location. This is the principle of trilateration.

If $X=\mathbb{R}^{3}$, then the preimages become spheres. In this case, four landmarks are needed: A pair of intersected spheres yields a circle, three spheres intersected yields two points, and four finally yield a unique point.

Filter 4 (Hyperbolic Positioning)

One shortcoming of trilateration is the precise clock synchronization needed to determine the signal time of arrival. Suppose that the towers are synchronized but the sensor is not. If the towers 


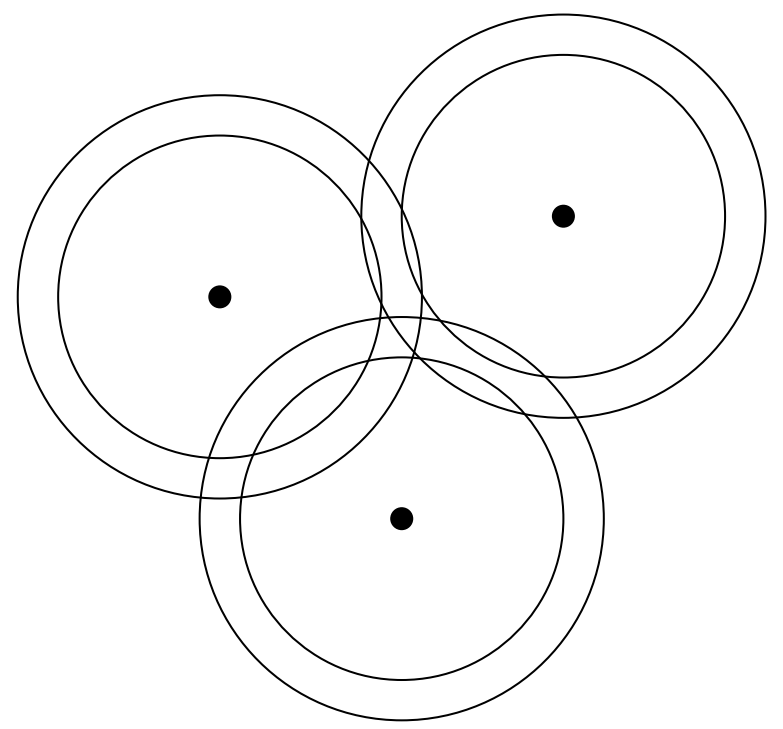

Figure 25: Disturbances lead to thicker preimages, which cause ambiguity in the triangulation.

send their signals at the same time, then the sensor can instead use a chronometer to measure the differences in their arrival times. This is called time difference of arrival (TDOA). If the signal propagation speed is known, then the virtual sensor yields the relative distances for each pair of towers. For example, it might tell us that Tower 1 is 432 meters closer to the sensor than Tower 2. What is the preimage in this case? The set of all points in the plane in which one tower is a fixed distance closer than another fall along a hyperbolic curve. The preimage in the three-dimensional case is one sheet of a hyperboloid. The method of hyperbolic positioning involves intersecting the hyperbolic preimages from multiple observations, one for each pair of towers, to obtain the precise location. This technique was used in the Decca Navigator System in World War II to locate ships, and in modern times it can be applied to localize a cell phone user using multiple cell towers.

How much can be learned about $x \in X$ from the observations? For any two sensors $h_{1}: X \rightarrow Y_{1}$ and $h_{2}: X \rightarrow Y_{2}$, consider their associated partitions $\Pi\left(h_{1}\right)$ and $\Pi\left(h_{2}\right)$. Let their observations be combined to produce one stronger sensor $h_{3}: X \rightarrow Y_{1} \times Y_{2}$, defined as $h_{3}(x)=\left(h_{1}(x), h_{2}(x)\right)$. In terms of partitions, $\Pi\left(h_{3}\right)$ is just the common refinement of $\Pi\left(h_{1}\right)$ and $\Pi\left(h_{2}\right)$. Furthermore, $h_{3}$ is just the greatest lower bound (glb) in the sensor lattice over $X$; recall Section 3.3.

Based on the filter models just presented, it is natural to wonder how information improves when sensors are combined. This depends on the properties of the preimages. Suppose, for example, that the sensor mappings are linear $y_{i}=C_{i} x$, with $Y=\mathbb{R}^{m_{i}}$ and $X=\mathbb{R}^{n}$. Each $h_{i}^{-1}\left(y_{i}\right)$ is a hyperplane through the origin of $X$, with its dimension depending on $m_{i}$ and the rank of $C_{i}$. Let every $C_{i}$ have full rank. If $m_{i}=n$, then $h_{i}^{-1}\left(y_{i}\right)$ indicates a unique $x \in X$ with a single observation. If $m_{i}=1$, then $\Delta\left(y_{1}, \ldots, y_{k}\right)$ produces a unique $x \in X$ only if $k=n$ and the $C_{i}$ column vectors are linearly independent. Moving away from this linear example, we generally consider nonlinear preimages. However, the properties of preimage dimension and sensor mapping independence remain critical to characterizing $\Delta\left(y_{1}, \ldots, y_{n}\right)$. 


\subsubsection{Handling disturbances}

In most applications of the triangulation principle, sensor observations are not perfectly predictable, even when the state is given. Therefore, it is crucial to consider disturbances. For the case of nondeterministic sensor mappings (20), the enlarged preimage (21) is obtained. See Figure 25. In that case, (40) is simply applied to the new preimages. One problem, however, is that the intersection of preimages might not be small enough to determine the state, regardless of how many observations are obtained. For example, suppose Filter 3 considers disturbances on the distance measured to the tower. If upper and lower bounds are specified on the distance, then each preimage is an annulus. If the distance error is at most $\epsilon>0$, then the annulus has thickness $2 \epsilon$. The intersection of several annuli will usually not result in a point, as shown in Figure 25.

This issue could motivate the consideration of disturbances probabilistically. The generalized triangulation principle can be adapted to the probabilistic case, in which the original set intersection is replaced by Bayes' rule. The probabilistic analog to (40) is

$$
p\left(x \mid y_{1}, \ldots, y_{n}\right)=\frac{p\left(y_{1} \mid x\right) p\left(y_{2} \mid x\right) \cdots p\left(y_{n} \mid x\right) p(x)}{p\left(y_{1}, \ldots, y_{n}\right)} .
$$

If we forget about making normalized probability density functions and define $p\left(y_{i} \mid x\right)=1$ if and only if $y_{i} \in h(x)$, then (41) is equivalent to (40). It can therefore be considered as an extension, assuming that such probabilities are realistically available.

In the probabilistic setting, it becomes reasonable to use many more observations than were minimally needed in Filters 1 to Filter 4. This allows as much information as possible to be applied to reduce ambiguity. In many problems the goal is to estimate the state $x$ that is producing the observations. Suppose $\hat{x}$ is a guess of the state. Let $d_{i}\left(\hat{x}, y_{i}\right)$ denote the Euclidean distance in $X$ from $\hat{x}$ to the nearest point on the preimage $h^{-1}\left(y_{i}\right)$. State estimation can now be formulated as an optimization problem: Try to find $\hat{x}$ that minimizes the distances $d_{i}$. How are all $d_{i}$ optimized simultaneously? One popular idea is to convert them to a scalar criterion, which often results in the least squares optimization problem:

$$
\min _{\hat{x} \in X} \sum_{i=1}^{n} d_{i}^{2}\left(\hat{x}, y_{i}\right) .
$$

This optimization is equivalent to maximum likelihood estimation in the probabilistic setting if the measurement errors are assumed to be distributed as zero-mean Gaussian densities. Otherwise, it can be simply viewed as a way to select $\hat{x}$ by reducing total error.

\subsubsection{Spatial filters over state-time space}

Recall from Section 3.4.3 that a sensor can also be defined over state-time space $Z$ to obtain $h: Z \rightarrow Y$. In this case, the concepts discussed so far extend naturally over time. We use the preimage definition (33) for triangulation in (40). See Figure 26. In this case, a spatiotemporal filter can be made that gains information simultaneously about state and time. An important example of this is GPS units. There is a difficult time synchronization problem, which makes it hard to use the simple trilateration model (Filter 3). GPS units therefore intersect preimages in $Z$ to infer both position and time, simultaneously. Minimally, one additional satellite is needed to sufficiently reduce the dimension of the set of possible $z=(x, t)$ values. This scheme generally requires five satellites; however, four is often cited the minimum number because the constraint that people stand on the earth is used to eliminate one. More satellites may be used, however, resulting in an optimization similar to (42) to overcome disturbances. 


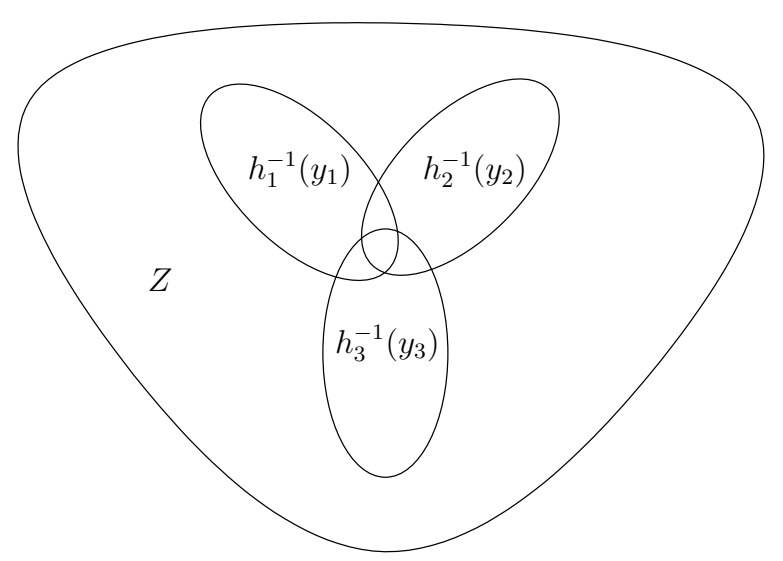

Figure 26: In state-time space, triangulation occurs by intersecting preimages in $Z$.

\subsection{Temporal Filters}

This section introduces filters that combine measurements over time.

\subsubsection{The Inference Problem}

Recall from Section 3.4.4 the notion of a state trajectory $\tilde{x}:[0, t] \rightarrow X$ and the space $\tilde{X}$ of all state trajectories. Now suppose that over the interval of time $[0, t]$, we have gathered and recorded sensor observations. If this could be done continuously, we obtain what would look like a "observation trajectory". Since observations may jump around erratically, rather than appearing as motions in the physical world, we instead call them the observation history, which is defined as a function $\tilde{y}:[0, t] \rightarrow Y$.

When presented with $\tilde{y}$, there are two fundamental questions:

1. What is the set of state trajectories $\tilde{x}:[0, t] \rightarrow X$ that might have occurred?

2. What is the set of possible current states, $\tilde{x}(t)$ ?

Using a single observation $y=h(\tilde{x}(t))$, we could answer the second question by the preimage $h^{-1}(y)$ from (2). However, based on an entire history $\tilde{y}$, we may able to further narrow down the set of possibilities. This will be the purpose of a temporal filter.

Before preceding to the filter details, we first introduce some concepts that help to illuminate the two fundamental questions above. Suppose that a sensor $h: X \rightarrow Y$ is given and is applied over an interval of time $[0, t]$. For every $t^{\prime} \in[0, t]$ some observation $\tilde{y}\left(t^{\prime}\right)=h\left(\tilde{x}\left(t^{\prime}\right)\right)$ is obtained. This means that we can use $h$, applied over $[0, t]$, to define a mapping

$$
H: \tilde{X} \rightarrow \tilde{Y}
$$

in which $\tilde{Y}$ is the set of all possible observation histories. For any sensor mapping $h: X \rightarrow Y$, the mapping $H$ is automatically induced.

To understand the two fundamental questions, we now only need to look at preimages of $H$. Compare this to $(2)$ :

$$
H^{-1}(\tilde{y})=\{\tilde{x} \in \tilde{X} \mid \tilde{y}=H(\tilde{x})\} .
$$

This is the "answer" to the first fundamental question: It yields the set of all state trajectories that could have produced $\tilde{y}$. Computing or even explicitly representing this answer could be extremely challenging. 


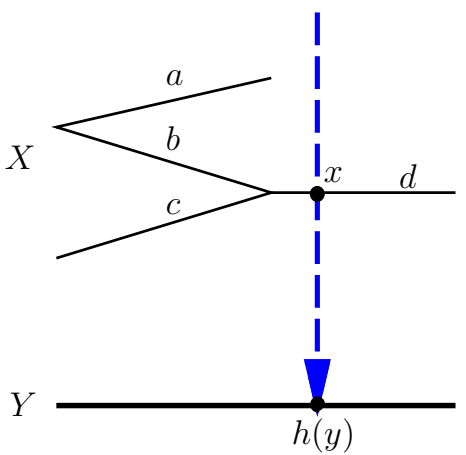

(a)

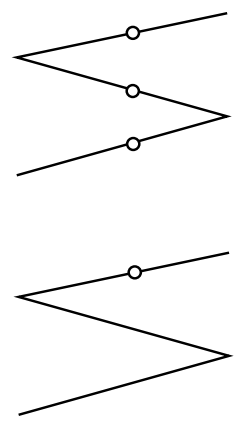

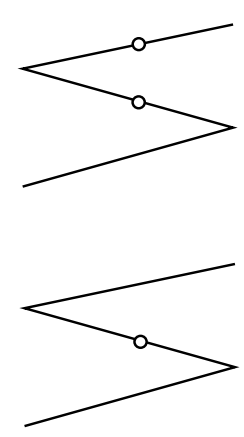
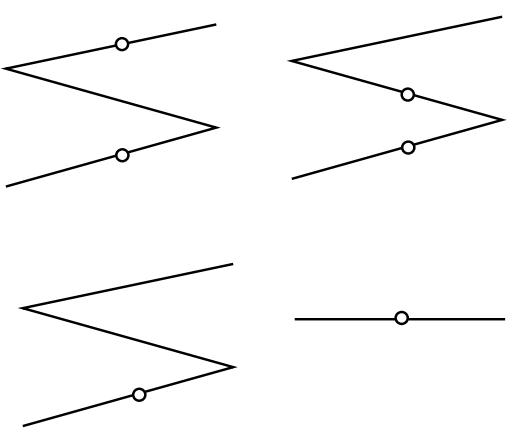

Figure 27: (a) Imagine trying to infer the location of a point on a planar graph while observing only a single coordinate. This simple example involves a point moving along a graph that has four edges. When the point is on the rightmost edge, there is no uncertainty; however, uncertainty exists when the point travels along the other edges. (b) The various possible I-states are shown, based on sensor preimages.

The second fundamental question can also be addressed using $H$. Here is one way to express the answer:

$$
\left\{x \in X \mid \exists \tilde{x} \in H^{-1}(\tilde{y}) \text { such that } \tilde{x}(t)=x\right\}
$$

A simple example will now be presented.

\section{Example 1}

Consider the upper part of Figure 27(a), in which there are four edges in an embedded planar graph that represents the state space $X \subset \mathbb{R}^{2}$. For convenience, the edges are labeled from $a$ to $d$. A point body travels around inside of the graph, and we would like to determine where it has gone using a simple sensor that measures only the horizontal coordinate. In other words, for any $p \in X$, with coordinates $p=\left(p_{1}, p_{2}\right)$, the sensor yields $y=h(x)=p_{1}$.

Consider the preimages of $h$ for various observations. If the body moves in edge $d$, then the state can be immediately inferred from the sensor observation. The preimage $h^{-1}(y)$ consists of a single point in $d$. If the body moves in the other edges, then $h^{-1}(y)$ contains three points, one inside of each of edge $a, b$, and $c$.

Now think about the two fundamental questions above. Imagine that the body has been moving around in $X$ for some time, and we want to reason about where it has been. Given $\tilde{y}:[0, t] \rightarrow Y$, what trajectories are possible, assuming the body must move on a continuous path? Any trajectory portion that places the body in edge $d$ can be completely determined from the observations. There is ambiguity only when the body is in other edges. If the body moves from edge $d$ to the left, then it can only enter edge $b$ or $c$. The observation preimage indicates that the body could be in $a, b$, or $c$; however, the history that the body came from $d$ eliminates the possibility of being in edge $a$. This nicely answers the second basic question, which is formulated mathematically in (45).

For the first question, we consider entire possible trajectories. For this simple problem, the set is always finite. For a simple example, imagine a trajectory that starts the body in the midpoint of $d$ at time $t=0$. The body then moves left to the midpoint of edge $b$ or $c$, arriving at time $t=1 / 2$. The body then returns to the right, reaching the midpoint of edge $d$ at time $t=1$. Based on $\tilde{y}:[0,1] \rightarrow Y$, the preimage $H^{-1}(\tilde{y})$, defined in (44), contains only two possible trajectories. One trajectory moves the body up to edge $b$ and then returns. The other moves the body down to 


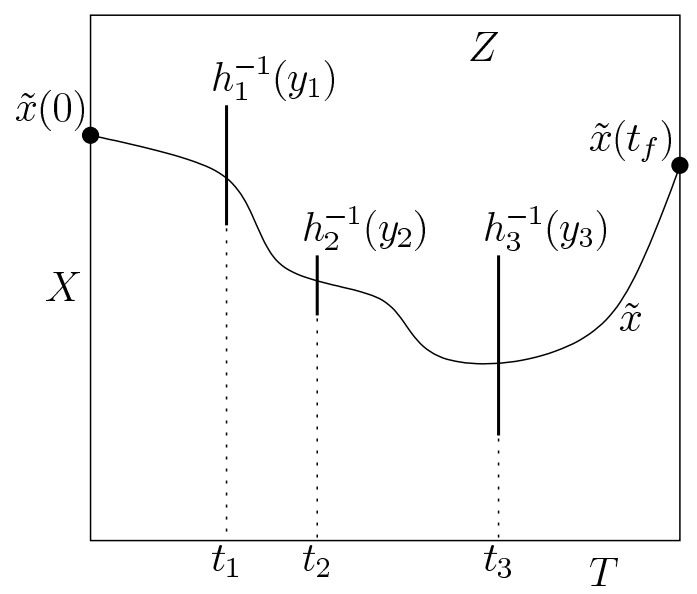

Figure 28: Each complete trajectory that is consistent with the observations must pass through the preimage of each observation, $y_{1}, y_{2}$, and $y_{3}$.

edge $c$ and returns. Note that the particular timing information is not ambiguous because using $\tilde{y}$, we know exactly which the times at which the body must be in various positions. The only ambiguity is which edge, $b$ or $c$, contains the body during part of the trajectory. If the sensor did not report all of the timing information, then $H^{-1}(\tilde{y})$ could be infinite. Imagine, for example, the possible trajectories if the sensor reports the horizontal position only once every 0.1 seconds. Any continuous trajectory segment is possible in between the observations.

In the spirit of Section 3.3, we can talk about the partition of $\tilde{X}$, denoted by $\tilde{\Pi}$, that is induced by $H$. Note that $\tilde{\Pi}\left(h_{1}\right)$ is a refinement of $\tilde{\Pi}\left(h_{2}\right)$ if and only if $h_{1} \succeq h_{2}$. This means that a more powerful sensor produces a better filter, which we would expect. Section 4.2.2 provides a more detailed construction of this.

\subsubsection{Triangulations over trajectory space}

The triangulation ideas from Section 4.1.1 can be extended over time. Recall the state-time space $Z=X \times T$ (Section 3.4.3) and the trajectory space $\tilde{X}$ (Section 3.4.4). It will be helpful to distinguish between complete and partial trajectories. Suppose $T=\left[0, t_{f}\right]$, in which $t_{f}$ is the final time. A complete trajectory is of the form $\tilde{x}: T \rightarrow X$ and a partial trajectory is of the form $\tilde{x}:[0, t] \rightarrow X$ for any $t \in\left[0, t_{f}\right)$. (We could alternatively allow unbounded trajectories: Define $T=[0, \infty)$ and allow any $t \in T$ to define a partial trajectory.) Let $\tilde{X}_{c}$ denote the set of complete trajectories.

Suppose that each sensor is of the form $h_{i}: Z \rightarrow Y$ and $y_{i}=h_{i}\left(x_{i}, t_{i}\right)=\left(y_{i}^{\prime}, t_{i}\right)$, in which $y_{i}^{\prime}=h_{i}^{\prime}(x)$ is a standard sensor mapping from Section 3.2.1. Here $x_{i}$ is the state at time $t_{i} \in T$, which can be written as $x_{i}=\tilde{x}\left(t_{i}\right)$ for the trajectory $\tilde{x}: T \rightarrow X$ in $\tilde{X}$. Essentially, $h_{i}$ is a sensor that indicates the precise time of each observation.

Suppose that $n$ observations, $y_{1}, \ldots, y_{n}$ are obtained, each of which is generated by $y_{i}=$ $h_{i}\left(\tilde{x}\left(t_{i}\right), t_{i}\right)$. What is the set of possible complete trajectories? Figure 28 illustrates the calculations. Each $y_{i}$ introduces a thin vertical window $h^{-1}\left(y_{i}\right) \subset Z$ at time $t_{i}$ through which a trajectory must travel to account for the observation. The set of possible complete trajectories are those that travel 
through all $n$ windows.

For a single observation $y_{i}=h_{i}\left(x_{i}, t_{i}\right)$, the set of possible complete trajectories is

$$
\tilde{h}_{i}^{-1}\left(y_{i}\right)=\left\{\tilde{x} \in \tilde{X}_{c} \mid \tilde{x}\left(t_{i}\right)=h_{i}\left(x_{i}, t_{i}\right)\right\}
$$

which is similar to (2). For the set of $n$ observations, we calculate the subset of $\tilde{X}_{c}$ of possible complete trajectories by

$$
\tilde{\triangle}\left(y_{1}, \ldots, y_{n}\right)=\tilde{h}_{1}^{-1}\left(y_{1}\right) \cap \tilde{h}_{2}^{-1}\left(y_{2}\right) \cap \cdots \cap \tilde{h}_{n}^{-1}\left(y_{n}\right),
$$

which can be considered as a form of triangulation, similar to (40). This can be interpreted as a filter that answers the second fundamental question of Section 4.2.1.

Since (46) and (47) appear to be preimages and triangulation, they can be once again viewed in a sensor lattice. However, the lattice is over $\tilde{X}_{c}$, the set of all complete trajectories. Each observation

partitions $\tilde{X}_{c}$ into class of trajectories that could account for it. The triangulation (47) applied to a pair of observations calculates a refinement of partitions, which is a lub in the lattice. Thus, this form of temporal filtering can be considered as traveling in a sensor lattice. In most settings, a unique trajectory will not be discovered due to time gaps in which no observations are made. Hence, the top of the lattice is not reached.

The second fundamental question of Section 4.2.1 is to determine the possible states at some time $t \in T$, assuming $t>t_{i}$ for all $i$ from 1 to $n$. This is expressed as

$$
\left\{x \in X \mid \exists \tilde{x} \in \tilde{\triangle}\left(y_{1}, \ldots, y_{n}\right) \text { with } \tilde{x}(t)=x\right\} .
$$

What was neglected above is the more complicated case in which perfect time stamps $t_{i}$ are not available with each $y_{i}$. In this case, the vertical bars at precise times in Figure 28 become "blobs" or more general regions in $Z$ because the times are unknown. For an observation $y \in Y$, any preimage in $Z$ and corresponding partition of $Z$ is possible. The temporal filter then uses the observations to construct a refinement of partitions over $Z$. This might not, however, induce a refinement of partitions over $\tilde{X}_{c}$ because one trajectory could be explained by multiple observation sets $y_{1}, \ldots$, $y_{n}$ (this was not the case when perfect time stamps were given). The next section considers an alternative model, in which sensor observations are received sequentially, but without time stamps. Furthermore, motion models are introduced to partially account for how the state may change over time when there are no observations.

\subsubsection{Temporal filters over discrete stages}

Discretely indexed observation histories Suppose that for some sensor model $h: X \rightarrow Y$, observations arrive at discretely over time, to yield a sequence $\tilde{y}=\left(y_{1}, \ldots, y_{k}\right)$ observations. Each $y_{i}$ will be said to correspond to a stage $i$. Depending on the particular model, it may or may not be known when each $y_{i}$ occurred; however, it will always be assumed that $y_{i+1}$ is obtained at a later time than $y_{i}$ for every $i$ from 1 to $k-1$.

In the previous model of observation histories $\tilde{y}:[0, t] \rightarrow Y$, the state was always being observed by the sensor. Under this new model, $\tilde{y}$ provides observations at some discrete points along time. In between these observations, the sensor does not make any other observations. Based on this situation, two distinguishing features of temporal filters arise:

1. Since observations arrive incrementally, we define filter information that can be updated incrementally. 
2. A model is needed of how the state might change over time, particularly when no observations are available.

It now becomes important to precisely define the information that will be stored internally during the filter operation. Let $\mathcal{I}$ be any set, and call it an information space ${ }^{4}$ (or I-space) and let any $\iota \in \mathcal{I}$ be called an information state (or I-state).

A temporal filter has two components:

1. A given initial I-state, denoted by $\iota_{0} \in \mathcal{I}$.

2. A transition function $\phi$ of the form $\phi: \mathcal{I} \times Y \rightarrow \mathcal{I}$. In particular, the structure is

$$
\iota_{k}=\phi\left(\iota_{k-1}, y_{k}\right),
$$

in which the new I-state $\iota_{k}$ is determined from the previous I-state $\iota_{k-1}$ and new observation $y_{k}$. When convenient, (49) will be shifted one stage forward to equivalently obtain $\iota_{k+1}=$ $\phi\left(\iota_{k}, y_{k+1}\right)$

Information states could have also been useful in Section 4.1 for spatial filters, but we were not concerned since all observations appeared simultaneously. Once observations are distributed over time, we must define what is stored internally.

Some generic, straightforward examples of temporal filters are now given.

Filter 5 (Sensor Feedback)

As a trivial special case, imagine a filter that maintains only the most recent observation. In this case $\mathcal{I}=Y$ and $\iota_{k}=\phi\left(\iota_{k-1}, y_{k}\right)=y_{k}$. Note that this filter does not even use the previous I-state $\iota_{k-1}$. This implies that it does not even require the initial I-state $\iota_{0}$, which has been left unspecified.

\section{Filter 6 (Stage Counter)}

Another simple example is to report the current stage. In this case, $\mathcal{I}=\mathbb{N} \cup\{0\}$, the set of nonnegative integers. We have $\iota_{0}=0$ and the filter is

$$
\iota_{k}=\iota_{k-1}+1 .
$$

Filter 7 (Simple State Estimator)

Suppose that $\mathcal{I}=X$ and the goal is to reconstruct the state from observations. To make the filter simple, suppose that $X=\mathbb{R}^{2}$ and a history-based sensor mapping is defined as

$$
y_{k}=h\left(x_{k}, x_{k-1}\right)=x_{k}-x_{k-1} .
$$

The initial I-state $\iota_{0}$ is the initial state, $\iota_{0} \in X$. The following filter perfectly recovers the state:

$$
\iota_{k}=\iota_{k-1}+y_{k} .
$$

By adding the observations, a telescoping sum is produced that results in $\iota_{k}=x_{k}$. Note that this filter refers back to the state space. Such reference is critical to most useful filters, and will be considered more carefully in Section 4.2.4.

\footnotetext{
${ }^{4} \mathrm{~A}$ more mathematically accurate name here would be information set because no topology is implied.
} 
Note that any filter can be extended to form a mapping over all observation histories. Suppose that $\tilde{y}_{k}=\left(y_{1}, \ldots, y_{k}\right)$ is provided. Starting with $\iota_{0}$, we can apply $\phi$ iteratively to obtain $\iota_{k}$. This implies that any filter (49), once given an initial I-state $\iota_{0}$, can be converted into a mapping $\kappa: \mathcal{I} \times \tilde{Y} \rightarrow \mathcal{I}$. This appears as

$$
\iota_{k}=\kappa\left(\iota_{0}, \tilde{y}_{k}\right) .
$$

We could more generally consider proposing any I-space $\mathcal{I}$ and any mapping $\kappa: \mathcal{I} \times \tilde{Y} \rightarrow \mathcal{I}$; however, in this section we are only interested in ones that can be constructed incrementally by iterating (49).

\subsubsection{Including motion models}

We would like to use a model about the evolution of states to help construct more informed filters. Filter 7 referred to the state, but did not need any assumptions about how states change.

We can introduce a state transition function, $f: X \rightarrow X$, which correctly predicts $x_{k+1}$ from $x_{k}$ with the equation $x_{k+1}=f\left(x_{k}\right)$. Let $X_{k}$ denote a given subset of $X$ that means the set of possible states at stage $k$. We will sometimes write $X_{k}(\ldots)$ to denote the set of possible states at stage $k$ based on all information inside of the parentheses. If the current state is not known, but is restricted to some subset $X_{k} \subseteq X$, then we can apply $f$ to every $x \in X_{k}$ to determine a forward projection:

$$
X_{k+1}\left(X_{k}\right)=\left\{x_{k+1} \in X \mid x_{k} \in X_{k} \text { and } x_{k+1}=f\left(x_{k}\right)\right\} .
$$

For many problems, perfect predictability is too restrictive. Perhaps we only know a set of possible future states. In this case, a nondeterministic state transition function is obtained, which is of the form $F: X \rightarrow \operatorname{pow}(X)$. The forward projection for this model becomes:

$$
X_{k+1}\left(X_{k}\right)=\left\{x_{k+1} \in X \mid x_{k} \in X_{k} \text { and } x_{k+1} \in F\left(x_{k}\right)\right\} .
$$

Probabilistic models could also be introduced, to obtain a probabilistic transition function $p\left(x_{k+1} \mid x_{k}\right)$. The forward projection in this case becomes a marginalization: $x_{k}$ is not actually given, but is replaced by some density $p\left(x_{k}\right)$. This yields:

$$
p\left(x_{k+1}\right)=\sum_{x_{k} \in X} p\left(x_{k+1} \mid x_{k}\right) p\left(x_{k}\right) .
$$

It could be the case that our characterization of future states depends on some actions chosen by ourselves or other bodies in the environment. Suppose that these actions are known and are recorded in the same way as a sensor observation. In this case, let $U$ be an action space and let $u_{k} \in U$ be an action applied at stage $k$. The particular $u_{k}$ simply becomes a new parameter in the state transition function. In the predictable case, we obtain $f: X \times U \rightarrow X$ and $x_{k+1}=f\left(x_{k}, u_{k}\right)$. In the nondeterministic case, we obtain $F: X \times U \rightarrow \operatorname{pow}(X)$. In the probabilistic case, we obtain $p\left(x_{k+1} \mid x_{k}, u_{k}\right)$. These are standard models used in control theory. The forward projections presented above naturally extend to allow $u_{k}$ to appear in the conditions.

It is assumed that one action is chosen at each stage. Let $\tilde{u}_{k}=\left(u_{1}, \ldots, u_{k}\right)$ be called an action history. The filter itself does not "chose" the actions. Whatever actions were applied by the system are merely reported as inputs to the filter. Choosing the actions is covered briefly in Section 5.1. The total information available at stage $k$ for constructing a filter in some I-space $\mathcal{I}$ is $\iota_{0}, \tilde{y}_{k}$, and $\tilde{u}_{k-1}$.

Taking the additional action information into account, the filter structure from (49) is extended to

$$
\iota_{k}=\phi\left(\iota_{k-1}, u_{k-1}, y_{k}\right)
$$


Filter 8 (History I-Space Filter)

One special kind of I-space will now be defined. Let $\eta_{k}=\left(\tilde{y}_{k}, \tilde{u}_{k-1}\right)$ be called the history I-state at stage $k$. It corresponds to all information available at stage $k$. Let $\mathcal{I}_{\text {hist }}$ be the set of all possible $\eta_{k}$ for all possible $k \geq 1$. For $k=1$, we obtain $\eta_{1}=\tilde{y}_{1}$ because there is no $u_{0}$. A trivial filter can be defined over $\mathcal{I}_{\text {hist }}$ as

$$
\eta_{k}=\phi\left(\eta_{k-1}, u_{k-1}, y_{k}\right) .
$$

In each iteration, the history I-state $\eta_{k-1}=\left(\tilde{u}_{k-1}, \tilde{y}_{k-1}\right)$ is simply extended to include the new information $u_{k-1}$ and $y_{k}$, which directly forms $\eta_{k}$. To initialize (58) properly, let $\eta_{0}=\emptyset$ and define the history I-space to include $\emptyset \in \mathcal{I}_{\text {hist }}$.

\subsubsection{Nondeterministic filters}

Once a state transition function has been determined, a generic filter can be defined that keeps track of the set of possible states at every stage, given all available information. For this filter, we define $\mathcal{I}_{n d e t}=\operatorname{pow}(X)$, in which $\mathcal{I}_{\text {ndet }}$ is called the nondeterministic I-space. Using all available information $\eta_{k}=\left(\tilde{u}_{k-1}, \tilde{y}_{k-1}\right)$ at stage $k$, we denote an I-state as $X_{k}\left(\eta_{k}\right)$, which is a subset of $X$. Thus, $X_{k}\left(\eta_{k}\right) \in \mathcal{I}_{n d e t}$.

Filter 9 (Generic Nondeterministic Filter)

See Section 11.2.2 of [37] for a detailed derivation and presentation of the filter appearing here. The general form of the generic nondeterministic filter is

$$
X_{k+1}\left(\eta_{k+1}\right)=\phi\left(X_{k}\left(\eta_{k}\right), u_{k}, y_{k+1}\right) .
$$

Let $X_{1} \subseteq X$ denote the initial I-state. We thus interpret $X_{1}$ as $\iota_{0}$, and $X_{1} \in \mathcal{I}_{\text {ndet }}$. The filter uses the sensor mapping $h: X \rightarrow Y$ and state transition function $F: X \times U \rightarrow \operatorname{pow}(X)$. After the first observation, the set of possible states is $X_{1}\left(y_{1}\right)=X_{1} \cap h^{-1}\left(y_{1}\right)$. In words, this simply intersects the initial possible states with the preimage due to $y_{1}$.

Now suppose inductively that $X_{k}\left(\eta_{k}\right)$ has been given, as appearing in (59). First consider taking into account $u_{k}$. This yields:

$$
X_{k+1}\left(\eta_{k}, u_{k}\right)=\bigcup_{x_{k} \in X_{k}\left(\eta_{k}\right)} F\left(x_{k}, u_{k}\right) .
$$

This can be considered as the set of all states that can be reached by starting from some state in $X_{k}\left(\eta_{k}\right)$ and applying an action $u_{k} \in U$. See Figure 29.

The next step is to take into account the observation $y_{k+1}$. This information alone indicates that $x_{k+1}$ lies in the preimage $h^{-1}\left(y_{k+1}\right)$. Therefore, an intersection is performed to obtain

$$
X_{k+1}\left(\eta_{k+1}\right)=X_{k+1}\left(\eta_{k}, u_{k}, y_{k+1}\right)=X_{k+1}\left(\eta_{k}, u_{k}\right) \cap h^{-1}\left(y_{k+1}\right) .
$$

This completes the detailed specification of (59).

After starting with the initial subset of $X$, the nondeterministic I-states at any stage can be computed by iterating (60) and (61) as many times as necessary. Note, however, that this generic filter may have high complexity or might not even be computable. This depends on the particular structure of the problem and on how the I-states are encoded; Section 4.3 will provide some settings in which the computations are simple and efficient. 

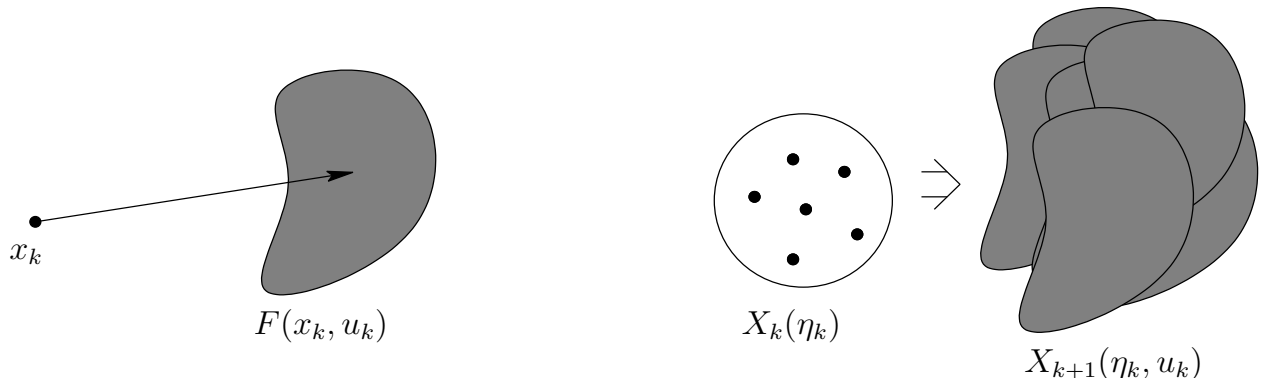

Figure 29: The first step in computing the nondeterministic I-state is to take the union of $F\left(x_{k}, u_{k}\right)$ over all possible $x_{k} \in X_{k}\left(\eta_{k}\right)$. It is the effect of applying an action when the current state is not precisely known.

\subsubsection{Probabilistic (Bayesian) filters}

A generic probabilistic (or Bayesian) filter can also be made. We define $\mathcal{I}_{\text {prob }}$ as the probabilistic $I$-space, which is the set of all probability density functions over $X$. It is assumed that such density functions exist (which might not be possible for some state spaces). Using $\eta_{k}$, we denote an I-state as $p\left(x_{k} \mid \eta_{k}\right)$ and note that $p\left(x_{k} \mid \eta_{k}\right) \in \mathcal{I}_{\text {prob }}$.

Filter 10 (Generic Probabilistic (Bayesian) Filter)

The filter takes the general form

$$
p\left(x_{k+1} \mid \eta_{k+1}\right)=\phi\left(p\left(x_{k} \mid \eta_{k}\right), u_{k}, y_{k+1}\right),
$$

which should be compared to the nondeterministic version (59).

To construct the filter, we use the sensor model $p\left(x_{k} \mid y_{k}\right)$ and the state transition model $p\left(x_{k+1} \mid x_{k}, u_{k}\right)$. The initial density, corresponding to $\iota_{0}$, is a given prior $p\left(x_{1}\right)$. The filter presented here is derived in Section 11.2.3 of [37] using the same notation. Other sources include [8, 31, 49].

Assume for now that $X$ is discrete. The filter (62) can be nicely expressed by first considering the effect of $u_{k}$, followed by $y_{k+1}$. Starting from $p\left(x_{k} \mid y_{k}\right)$ and using $u_{k}$ in the state transition model, we obtain

$$
\begin{aligned}
p\left(x_{k+1} \mid \eta_{k}, u_{k}\right) & =\sum_{x_{k} \in X} p\left(x_{k+1} \mid x_{k}, u_{k}, \eta_{k}\right) p\left(x_{k} \mid \eta_{k}\right) \\
& =\sum_{x_{k} \in X} p\left(x_{k+1} \mid x_{k}, u_{k}\right) p\left(x_{k} \mid \eta_{k}\right) .
\end{aligned}
$$

Taking this result and applying the sensor model on $y_{k+1}$ yields

$$
p\left(x_{k+1} \mid y_{k+1}, \eta_{k}, u_{k}\right)=\frac{p\left(y_{k+1} \mid x_{k+1}, \eta_{k}, u_{k}\right) p\left(x_{k+1} \mid \eta_{k}, u_{k}\right)}{\sum_{x_{k+1} \in X} p\left(y_{k+1} \mid x_{k+1}, \eta_{k}, u_{k}\right) p\left(x_{k+1} \mid \eta_{k}, u_{k}\right)} .
$$

If $X$ is not discrete, then the summations above are replaced with integrals.

Once again, there may be considerable computational challenges when implementing these filters. Many sampling-based techniques, such as particle filters, have been developed and used in practice to implement them. For their application in robotics, see [49].

For some problems, the probabilistic I-space collapses nicely and the computations become efficient. 
Filter 11 (Kalman Filter)

The most famous and useful example of this is the Kalman filter. It can be considered as a specialized version of Filter 10 that just happens to work out efficiently. In the special case in which both $f$ and $h$ are linear functions, and $p(\theta), p(\psi)$, and $p\left(x_{1}\right)$ are Gaussian, all probabilistic filter I-states become Gaussian. This means that the probabilistic I-space, $\mathcal{I}_{\text {prob }}$, does not need to represent every conceivable probability density function. The filter I-state is always trapped in the subspace of $\mathcal{I}_{\text {prob }}$ that corresponds only to Gaussians. The subspace is denoted as $\mathcal{I}_{\text {gauss }}$.

The motion model is called linear-Gaussian (or $L G$ ). Each Gaussian density on $\mathbb{R}^{n}$ is fully specified by its $n$-dimensional mean vector $\mu$ and an $n \times n$ symmetric covariance matrix, $\Sigma$. Therefore, $\mathcal{I}_{\text {gauss }}$ can be considered as a subset of $\mathbb{R}^{m}$ in which $m=2 n+\left(\begin{array}{c}n \\ 2\end{array}\right)$. For example, if $X=\mathbb{R}^{n}$, then $\mathcal{I}_{\text {gauss }} \subset \mathbb{R}^{5}$, because two independent parameters specify the mean and three independent parameters specify the covariance matrix (not four, because of symmetry).

The full Kalman filter is expressed below, but the full derivation, which is lengthy but not difficult and can be found in virtually any textbook on stochastic control (e.g., [4, 31]).

All of the required spaces are Euclidean, but they may have different dimensions. Therefore, let $X=\mathbb{R}^{n}, U=\Theta=\mathbb{R}^{m}$, and $Y=\Psi=\mathbb{R}^{r}$. Let $A_{k}, B_{k}, C_{k}, G_{k}$, and $H_{k}$ each denote a matrix with constant real-valued entries and which may or may not be singular. The matrices are assumed to have appropriate dimensions for the multiplications below. The $k$ subscript is used to indicate that a different matrix may be used in each stage. In many applications, the matrices will be the same in each stage.

The motion model in this setting becomes

$$
x_{k+1}=f_{k}\left(x_{k}, u_{k}\right)=A_{k} x_{k}+B_{k} u_{k}+G_{k} \theta_{k},
$$

The notation $f_{k}$ is used because the model can vary with stages.

The sensor mapping $y_{k}=h_{k}\left(x_{k}, \psi_{k}\right)$ is

$$
y_{k}=C_{k} x_{k}+H_{k} \psi_{k}
$$

Note that with $C_{k}$, the sensor output may also vary with stages.

In each stage, disturbance parameters $\theta_{k}$ and $\psi_{k}$ are modeled with zero-mean Gaussians. Thus, each has an associated covariance matrix, denoted by $\Sigma_{\theta}$ and $\Sigma_{\psi}$, respectively. Using the model given so far and starting with an initial Gaussian density over $X$, all resulting I-states will be Gaussian [31].

Since every I-state in $\mathcal{I}_{\text {gauss }}$ can be represented by a mean and covariance, let $\mu_{k}$ and $\Sigma_{k}$ denote the mean and covariance of $p\left(x_{k} \mid \eta_{k}\right)$. The expressions given in the remainder of this section define a derived information transition equation that computes $\mu_{k+1}$ and $\Sigma_{k+1}$, given $\mu_{k}, \Sigma_{k}, u_{k}$, and $y_{k+1}$. The process starts by computing $\mu_{1}$ and $\Sigma_{1}$ from the initial conditions.

Assume that an initial condition is given that represents a Gaussian density over $\mathbb{R}^{n}$. Let this be denoted by $\mu_{0}$, and $\Sigma_{0}$. The first I-state, which incorporates the first observation $y_{1}$, is computed as $\mu_{1}=\mu_{0}+L_{1}\left(y_{1}-C_{1} \mu_{0}\right)$ and

$$
\Sigma_{1}=\left(I-L_{1} C_{1}\right) \Sigma_{0}
$$

in which $I$ is the identity matrix and

$$
L_{1}=\Sigma_{0} C_{1}^{T}\left(C_{1} \Sigma_{0} C_{1}^{T}+H_{1} \Sigma_{\psi} H_{1}\right)^{-1} .
$$

Although the expression for $L_{1}$ is complicated, note that all matrices on the right were given. 
Now that $\mu_{1}$ and $\Sigma_{1}$ have been expressed, the base case is completed. The next part is to give the iterative updates from stage $k$ to stage $k+1$. Using $\mu_{k}$, the mean at the next stage is computed as

$$
\mu_{k+1}=A_{k} \mu_{k}+B_{k} u_{k}+L_{k+1}\left(y_{k+1}-C_{k+1}\left(A_{k} \mu_{k}+B_{k} u_{k}\right)\right)
$$

in which $L_{k+1}$ will be defined shortly. The covariance is computed in two steps; one is based on applying $u_{k}$, and the other arises from considering $y_{k+1}$. Thus, after $u_{k}$ is applied, the covariance becomes

$$
\Sigma_{k+1}^{\prime}=A_{k} \Sigma_{k} A_{k}^{T}+G_{k} \Sigma_{\theta} G_{k}^{T} .
$$

After $y_{k+1}$ is received, the covariance $\Sigma_{k+1}$ is computed from $\Sigma_{k+1}^{\prime}$ as

$$
\Sigma_{k+1}=\left(I-L_{k+1} C_{k+1}\right) \Sigma_{k+1}^{\prime} .
$$

The expression for $L_{k}$ is

$$
L_{k}=\Sigma_{k}^{\prime} C_{k}^{T}\left(C_{k} \Sigma_{k}^{\prime} C_{k}^{T}+H_{k} \Sigma_{\psi} H_{k}\right)^{-1} .
$$

To obtain $L_{k+1}$, substitute $k+1$ for $k$ in (72). Note that to compute $\mu_{k+1}$ using (69), $\Sigma_{k+1}^{\prime}$ must first be computed because (69) depends on $L_{k+1}$, which in turn depends on $\Sigma_{k+1}^{\prime}$.

Although the expressions above appear complicated, note the simple structure of the Kalman filter:

$$
\left(\mu_{k+1}, \Sigma_{k+1}\right)=\phi\left(\left(\mu_{k}, \Sigma_{k}\right), u_{k}, y_{k+1}\right)
$$

Due to the collapsing I-space and efficient updates, the Kalman filter provides a beautiful solution to the class of linear Gaussian models.

It would be wonderful if the same type of simplification that took us from general Bayesian filters to the Kalman filter were possible for the case of nondeterministic filters. It turns out that there are many circumstances in which analogous behavior occurs. This is the subject of Section 4.3.

\subsection{Combinatorial Filters}

This section adapts the nondeterministic filter (Filter 9) to a family of problems for which the computations become very efficient. By careful choice of models, the computation step illustrated in Figure 29 can be computed in an exact, combinatorial way. This leads to a family called combinatorial filters.

\subsubsection{Obstacles and beams}

This topic is adapted from [50]. Consider what information can be obtained from the knowledge that one or more moving bodies pass through detection beams. See Figure 30(a). An unpredictable point body moves inside of a given polygonal region $E$ in the plane. The state space is $X=E \subset \mathbb{R}^{2}$. There are several static binary detectors, as in Model 14, and for each the detection region $V$ is a line segment for which each endpoint connects to $\partial E$ (the boundary of $E$ ). Each detector is called a beam and is uniquely labeled $a, b, c$, and so on. Rather than output 0 or 1 as in (11), we modify $h$ so that each outputs 0 or its own label instead of 1 . We can combine the beams into a single sensor that produces a label whenever the corresponding beam is crossed. If the body is not touching any beams, then the combined sensor continuously yields 0 . An observation history $\tilde{y}$ will therefore be compressed to indicate the sequence of beams that were crossed over time. For example $\tilde{y}=$ cbabdeeefe for the state trajectory in Figure 30(a). 


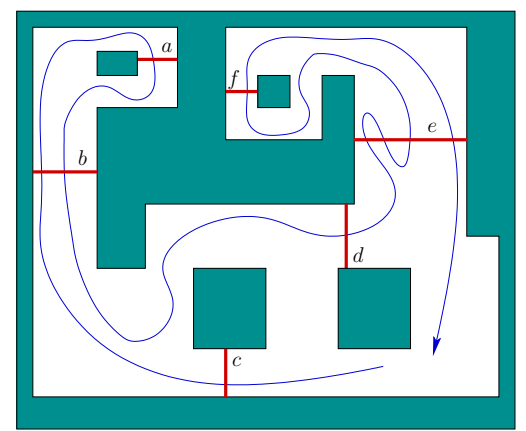

a.

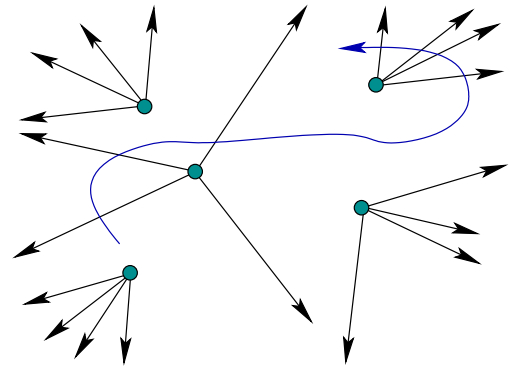

b.

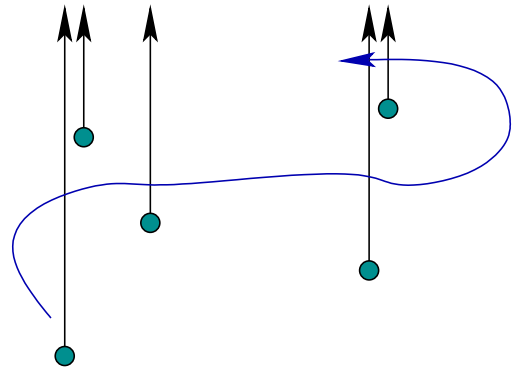

c.

Figure 30: (a) Imagine placing some beam-shaped detection sensors around an indoor environment. (b) Virtual beams could even arise by noticing when towers become aligned in an image while exploring a field. (c) Alternatively, virtual beams could arise from a robot using a compass: A virtual beam is crossed whenever a target is directly north.

There are many ways to physically implement this model. We could, for example, use the safety beams shown in Figure 4(h). We could even obtain "virtual beams" through an entirely different physical sensor model. For example, Figure 30(b) shows 5 landmarks (imagine them as towers) in the plane. If a robot moves in the plane and uses a camera, it could detect when a pair of landmarks is perfectly aligned. This would correspond to having beams placed as shown in the figure. Alternatively, Figure 30(c) shows how virtual beams would appear if a robot has a compass and can determine precisely when a landmark is directly south of its position.

Now think about what can be inferred after receiving a sequence of beam crossings. Clearly, the sensing model is too weak to recover the state. For the example in Figure 31(a), suppose that the beams divide $E$ into three two-dimensional regions, $r_{1}, r_{2}, r_{3}$. Let $R=\left\{r_{1}, r_{2}, r_{3}\right\}$. For this simple example, if the initial region is given, then the new region can be determined after each beam is crossed. This assumes that the body never touches a beam without crossing it completely.

Filter 12 (Simple Region Filter)

Consider any environment $E \subseteq \mathbb{R}^{2}$ in which a finite collection of beams is arranged so that: 1) every beam either touches $\partial E$ at each end or shoots off to infinity, 2) every beam is uniquely labeled, 3) no pair of beams intersects. Let $R$ be the set of two-dimensional regions formed by taking the maximal connected regions that can be traversed by the body without crossing a beam (this was applied to generate the three regions in Figure 31(a)).

For this general problem, we can make a simple filter that keeps track of the current region, assuming it was known initially. Let $\mathcal{I}=R$ and specify $\iota_{0}=r_{0}$ as the initial region. Using the filter template (49), we obtain $\phi: R \times Y \rightarrow R$ and

$$
r_{k}=\phi\left(r_{k-1}, y_{k}\right) \text {. }
$$

The next region $r_{k}$ can be easily determined once $r_{k-1}$ and $y_{k}$ (the most recent beam crossed) are given.

We now consider a more complicated problem, such as the one shown in Figure 31(b). Suppose that the initial region is not known. Furthermore, we will allow a much more complicated collection of beams. We still require all beams to have linear detection regions for which both ends reach $\partial E$. However, beams may or may not have each of the following three properties: 


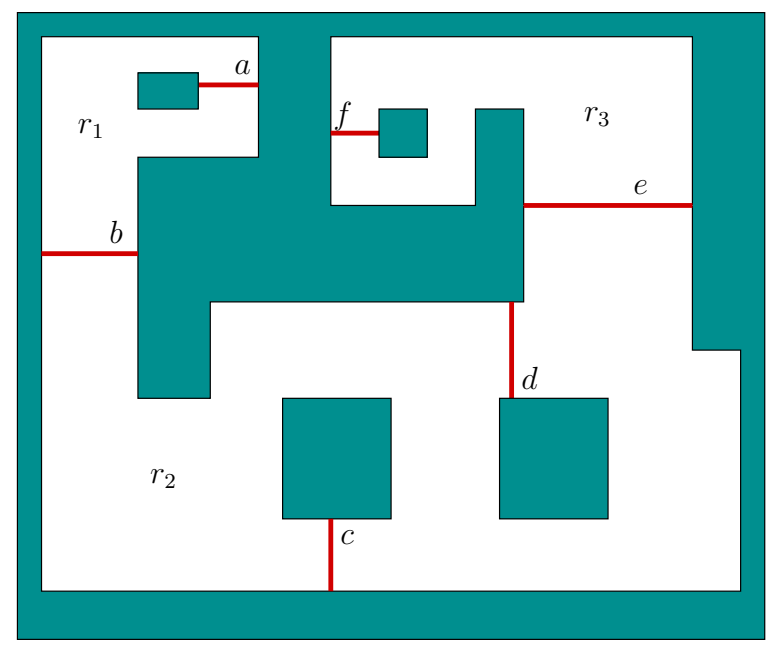

a.

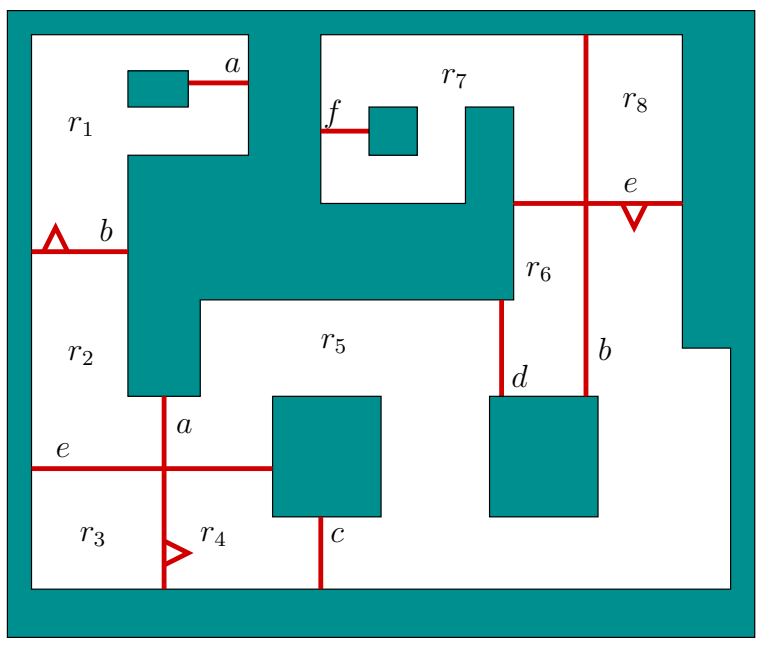

b.

Figure 31: (a) A simple example, which leads to three regions, $r_{1}, r_{2}$, and $r_{3}$. (b) Beams may be directional, may intersect, and may be indistinguishable.

1. Beams may or may not be distinguishable. Two or more beams could produce the same label.

2. Beams may or may not be disjoint. In other words, a pair of beams may intersect in the interior of $E$.

3. Beams may or may not be directed. If a beam is directed, then we know which direction that body went when crossing. The beams in Figure 31(a) were undirected. For some beams in Figure 31(b), the beam's natural direction is shown with a triangular pointer placed on the beam. A body may traverse such beams in the natural direction or in the opposite direction. This information can be observed. For example, we may receive $a$ if beam $a$ is crossed in the natural direction, or receive $a^{\prime}$ for crossing in the opposite direction.

In spite of this complicated problem, the set $R$ of regions can be defined as before. Each $r \in R$ is a connected two-dimensional region in $\mathbb{R}^{2}$ in which the body can travel without crossing a beam. We define a multigraph $G$ as follows. Every vertex in $G$ corresponds to a region in $\mathbb{R}^{2}$. A directed edge is made from $r_{1} \in R$ to $r_{2} \in R$ if and only if the body can cross a single beam to go from $r_{1}$ to $r_{2}$. The beam label is placed on the edge. If the beam is undirected, then directed edges are made in both directions with the same label. If beam $a$ is directed, then the label is $a$ in one direction and $a^{\prime}$ in the other. Note that a self-loop edge may be formed if a beam can be crossed while remaining in the same region. Figure 32 shows a simple example in which there are two beams and two regions.

Filter 13 (Nondeterministic Region Filter)

We now describe a filter that keeps track of the set of possible current regions. It can be considered as an special case of Filter 9 for which the nondeterministic I-space has structure that makes the filter easy to compute. Let $\mathcal{I}=\operatorname{pow}(R)$, and $\iota_{0}=R_{0}$, a set of possible initial regions. The multigraph $G$ is given (it can be computed from a description of $E$ and the beam locations). A simple example is shown in Figure 32.

The method keeps track of possible regions by marking the corresponding vertices of $G$. Initially, mark every vertex in $R_{0}$; all other vertices are cleared. The filter proceeds inductively, yielding $R_{k+1}=\phi\left(R_{k}, y_{k+1}\right)$. At stage $k$, the marked vertices are precisely those corresponding to $R_{k}$. 


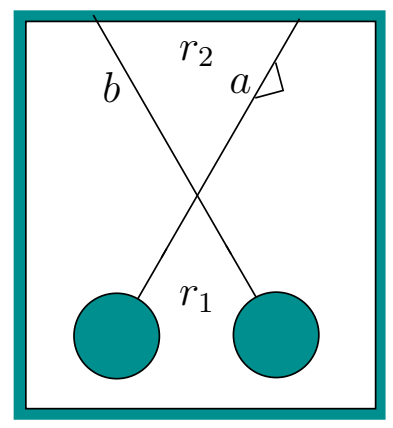

a.

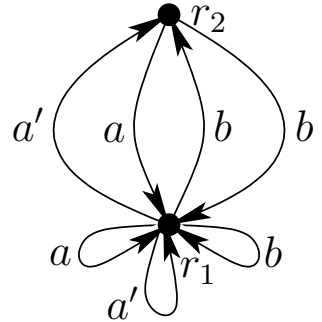

b.

Figure 32: (a) An example that has two intersecting beams, one directed and one undirected. (b) The corresponding multigraph $G$.

Suppose that $y_{k+1}$ is observed, which extends the sensor word by one observation. For each marked vertex, look for any outgoing edge labeled with $y_{k+1}$. For each one found, the destination vertex is marked. Any vertex that was marked at stage $k$ but did not get marked in stage $k+1$ becomes cleared. Note that the total number of marked vertices may increase because from a single vertex there may be multiple edges that match $y_{k+1}$. Also, this approach works for the case of partially distinguishable beams because the match is based on the observation $y_{k+1}$, rather than the particular beam. The set of marked vertices yields $R_{k+1}$.

Filter 14 (Multiple Body Filter)

What if there are multiple bodies moving in $E$ ? Filter 13 could be generalized to keep track of which room each body might be in. Consider the example in Figure 33 in which two bodies move in an annulus-shaped environment $E$. The state space is $X=E^{2} \subset \mathbb{R}^{4}$. Since there are two agents, there are 9 possible combinations: $(1,1),(1,2),(1,3),(2,1),(2,2),(2,3),(3,1),(3,2)$, and $(3,3)$. In terms of the filter template (49), we let $\mathcal{I}=\operatorname{pow}(R \times R)$. Initially, $\iota_{0}$ is given, which is the set of possible initial room combinations. For example, someone may tell us that they are both in $R_{1}$, in which case $\iota_{0}=\{(1,1)\}$.

For simplicity here, assume that beams cannot be crossed simultaneously by two bodies. To make the filter, we can directly extend the method of Filter 13 . Let $G^{2}$ be the multigraph formed by taking the Cartesian product $G \times G$ in the sense that the vertices correspond to all ordered pairs of regions. Each edge in $G^{2}$ is formed if a transition from one ordered pair to another is possible after a single observation $y_{k+1}$. Once $G^{2}$ is formed, the method of propagating markers over the vertices, used in Filter 13, can be adapted and used here. In each iteration, the set of possible region pairs is maintained.

If there are $n$ bodies in the environment, then the method can be extended by forming an $n$-fold Cartesian product of $R$ to obtain $\mathcal{I}$ and a $n$-fold product of $G$ to obtain $G^{n}$. If the number of bodies is unknown but bounded by $n$, then the disjoint union of I-spaces and $G^{i}$ is formed, for each $i$ from 0 to $n$. If the number is bodies is unbounded, then the method as described fails.

Of course the scheme described in Filter 14 is computationally prohibitive if there are many bodies and regions. Asymptotically, the number of vertices in $G^{n}$ is exponential in $n$. There are many opportunities to dramatically reduce the filter complexity depending on the particular information of interest. This remains an open research problem: Determine what information can 


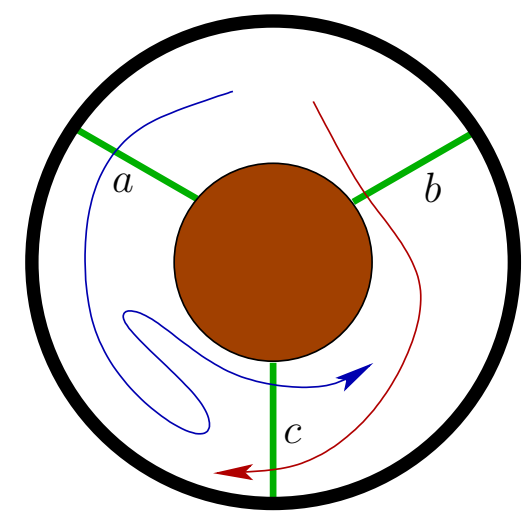

a.

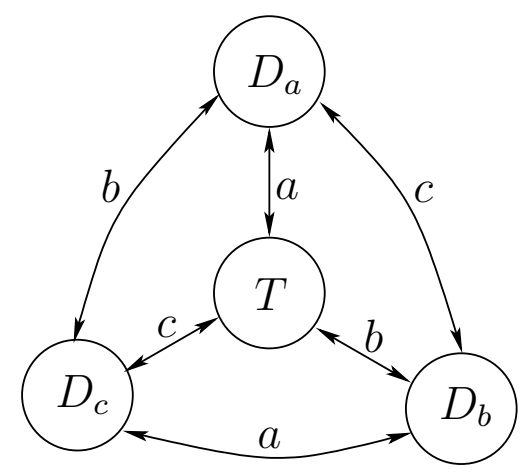

$\mathrm{b}$.

Figure 33: (a) Two bodies move in an environment consisting of three regions and three undirected beams. (b) A simple filter with only four I-states is sufficient to keep track of whether the two bodies are together in some region.

be maintained by a region filters that have much lower complexity. This is similar to the task of minimizing automata using Nerode equivalence classes in the classical theory of computation [26].

Filter 15 (Two-Bit Filter)

A simple example is presented in Figure 33. Two bodies move in a simple environment and we are interested only in one particular question: Are the two bodies together in a region, or are they separated by a beam? It turns out that a particular filter can be designed for this question, and it is dramatically simpler than the generic one from Filter 14 . The I-space is $\mathcal{I}=\left\{T, D_{a}, D_{b}, D_{c}\right\}$ and the filter is depicted in Figure 33(b). The $T$ I-state means that the bodies are together. Each $D_{x}$ I-state means that they are in neighboring regions and are separated by beam $x$. The automaton in Figure 33(b) defines the filter $\iota_{k}=\phi\left(\iota_{k-1}, y_{k}\right)$ by indicating the transitions caused by each specific $y_{k}$. If the initial I-state is given, then this simple "two-bit" filter can always, correctly answer the question about whether the two bodies are together (represented by the $T$ I-state).

Filter 15 dramatically reduces the complexity of Filter 14 by finding the perfect I-space. How can this be done for other problems? Many exciting problems for future research remain!

In addition to trying to simplify the region filters, we might also want to make them more robust by tolerating disturbances in the beams. There may be false positives and false negatives, as described in Model 31 (faulty detectors). If we learned probabilistic models of these disturbances, then Filter 13 can be adapted to the probabilistic case. The I-space is the set of all probability density functions over $R$. This would follow the template of Section 4.2.6, resulting in a probabilistic filter (Filter 10) that starts with a prior $p\left(r_{1}\right)$ and iteratively computes $p\left(r_{k+1} \mid \tilde{y}_{k+1}\right)$ using only $p\left(r_{k} \mid \tilde{y}_{k}\right)$ and $y_{k+1}$. At each stage, the filter maintains a probability density over the possible regions that contain the body, based on the entire observation history.

\subsubsection{Shadow information spaces}

This topic is adapted from [53]. For these filters, imagine that several robots carry detection sensors (from Section 3.2.5) and move through a common environment that contains numerous moving point bodies, as shown in Figure 34. Suppose that $q$ represents the configuration of all robots and 


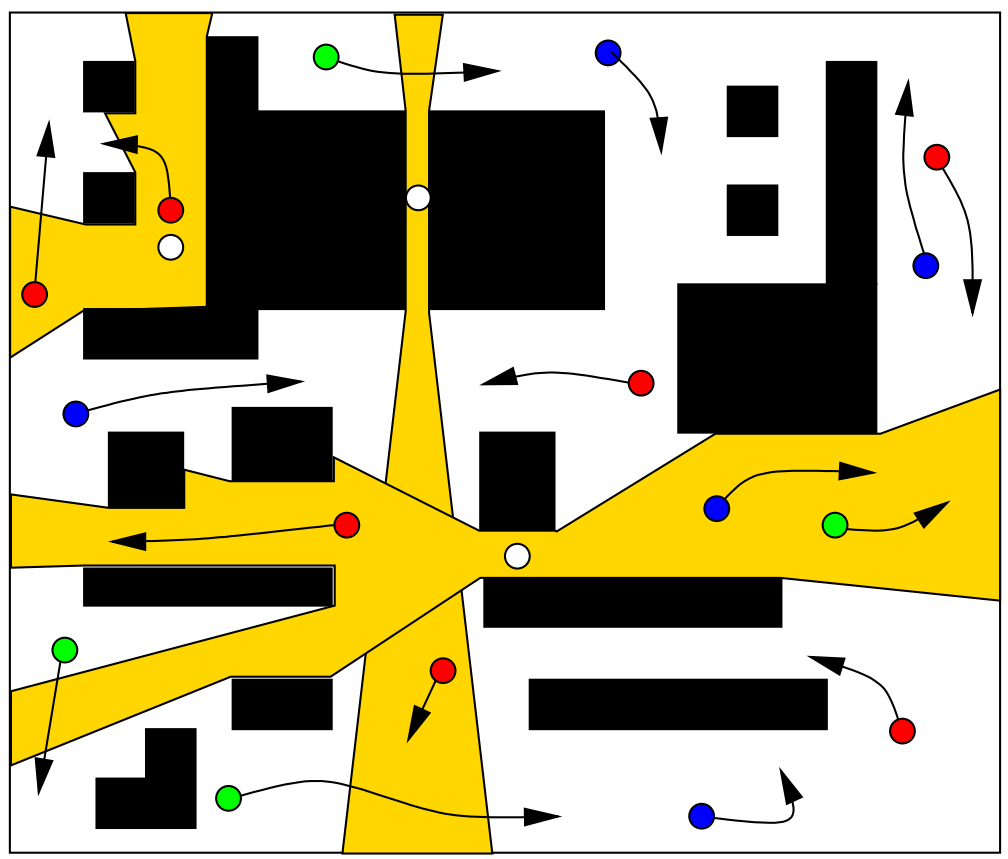

Figure 34: Imagine trying to keep track of bodies outside of the field of view of sensors. These regions, shown in white, are called shadows. This example has nine shadows.

$V(q) \subseteq E$ is their combined detection region in some environment $E$. The topic of interest is the part of $E$ that is not visible to the sensors at a particular instant. We define $S(q)=E \backslash V(q)$ and call it the shadow region. See Figure 35. Assume that if any body enters $V(q)$ it is detected by the sensors. Now think about portions of $S(q)$ within which bodies become trapped: Within any connected component of $S(q)$, a body cannot leave without being detected. We therefore consider a family of filters based on analyzing the connected components of the shadow region $S(q)$ and how they change over time.

A shadow region can generally be partitioned into a finite set of such components, called shadow components. As the robots move, the particular $S(q)$ gradually changes. The only changes of interest to us are the following combinatorial events:

1. Disappear: A shadow component vanishes, which eliminates a hiding place for the bodies.

2. Appear: A shadow component appears, which introduces a new hiding place for the bodies.

3. Split: A shadow component splits into multiple shadow components.

4. Merge: Multiple shadow components merge into one shadow component.

These are the only events that will concern us. To keep this paper simple, assume that: 1) no two events occur simultaneously, 2) a shadow component splits into at most two components, and 3) at most two components may merge into one. The events are illustrated in Figure 36.

Each time period over which no combinatorial events occur can be referred to as a stage. During a stage, if the particular shape or size of a shadow component varies, it will not be of interest. Each shadow component during a stage will be denoted $s$, and an entire set of $n$ shadow components at stage $k$ is denoted

$$
S_{k}=\left\{s_{1}, s_{2}, \ldots, s_{n}\right\}
$$




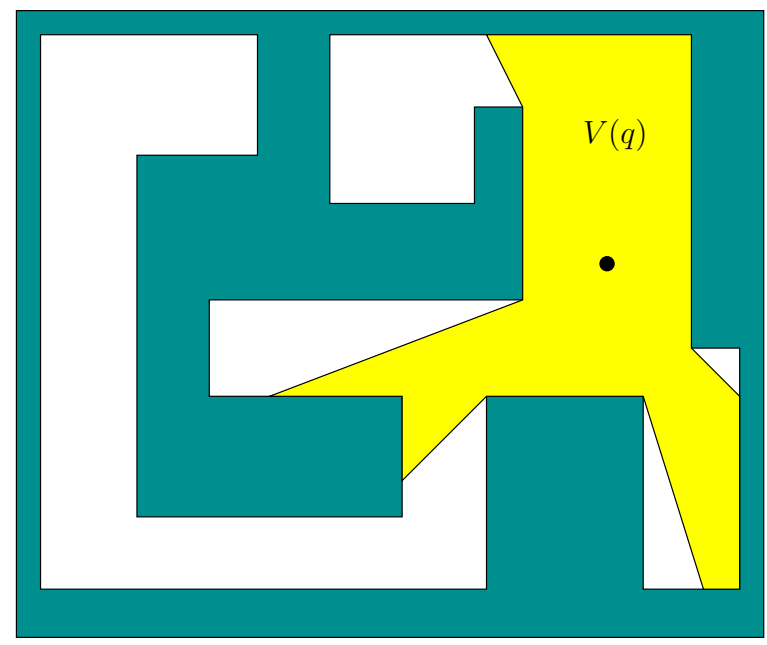

a.

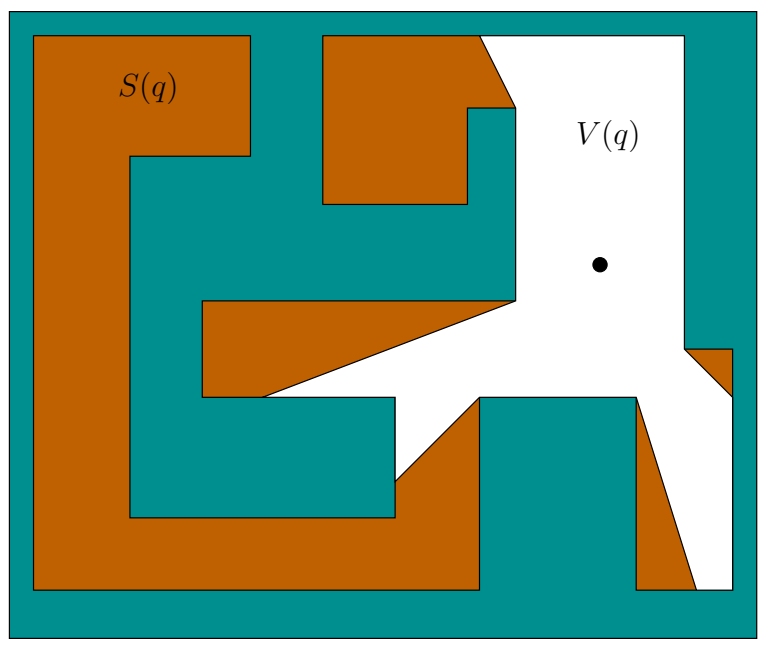

b.

Figure 35: (a) The detection region for a single robot that carries a detection sensor. (b) The corresponding shadow region, which has 5 connected components.

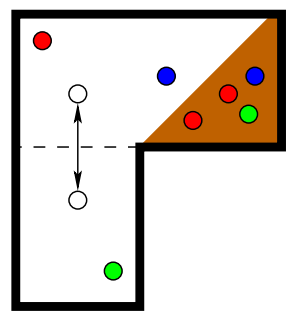

a.

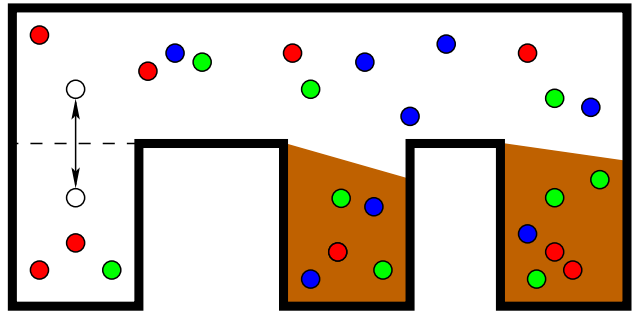

b.

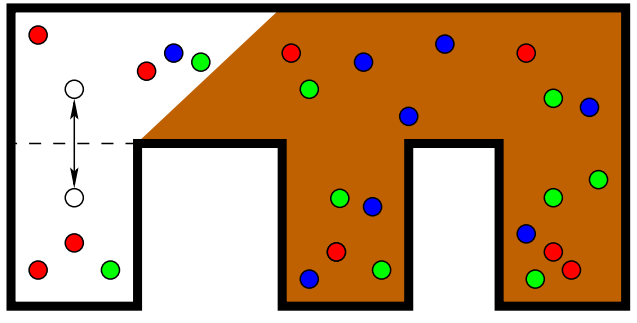

c.

Figure 36: (a) A disappear or appear event occurs, depending on the direction of motion. (b,c) A split or merge event is illustrated, depending on the direction of motion.

We start with $S_{1}$ as the initial shadow components. Based on a combinatorial event, we obtain a transition from $S_{k}$ to $S_{k+1}$. Typically, most components remain unchanged. For the disappear event, $S_{k+1}=S_{k} \backslash\{s\}$ for some component $s$. For an appear event, some new $s$ is used to obtain $S_{k+1}=S_{k} \cup\{s\}$. For the split and merge events, three components become involved in the change. Let $M\left(s, s^{\prime}, s^{\prime \prime}\right)$ denote a merge relation, which is true when $s$ and $s^{\prime}$ merge to form $s^{\prime \prime}$. Likewise, let $S\left(s, s^{\prime}, s^{\prime \prime}\right)$ denote a split relation, which is true when $s$ splits to form $s^{\prime}$ and $s^{\prime \prime}$. Figure 37 shows a simple example.

We call the complete sequence $\left(S_{1}, \ldots, S_{k}\right)$, together with the specification of the split and merge relations, a shadow sequence I-state, and denote it by $\sigma_{k}$. Let $\mathcal{I}_{\text {sseq }}$ denote the shadow sequence I-space, which corresponds to all possible $\sigma_{k}$ for a given problem. We assume that for whatever given problem, the mapping from $\mathcal{I}_{\text {hist }}$ to $\mathcal{I}_{\text {sseq }}$ exists and can be applied to yield $\sigma_{k}$ from the observation and action histories.

We now define some filters that keep track of information over $\mathcal{I}_{\text {sseq }}$.

Filter 16 (Pursuit-Evasion Filter)

Suppose we would like to keep track of whether each shadow component is known not to contain 


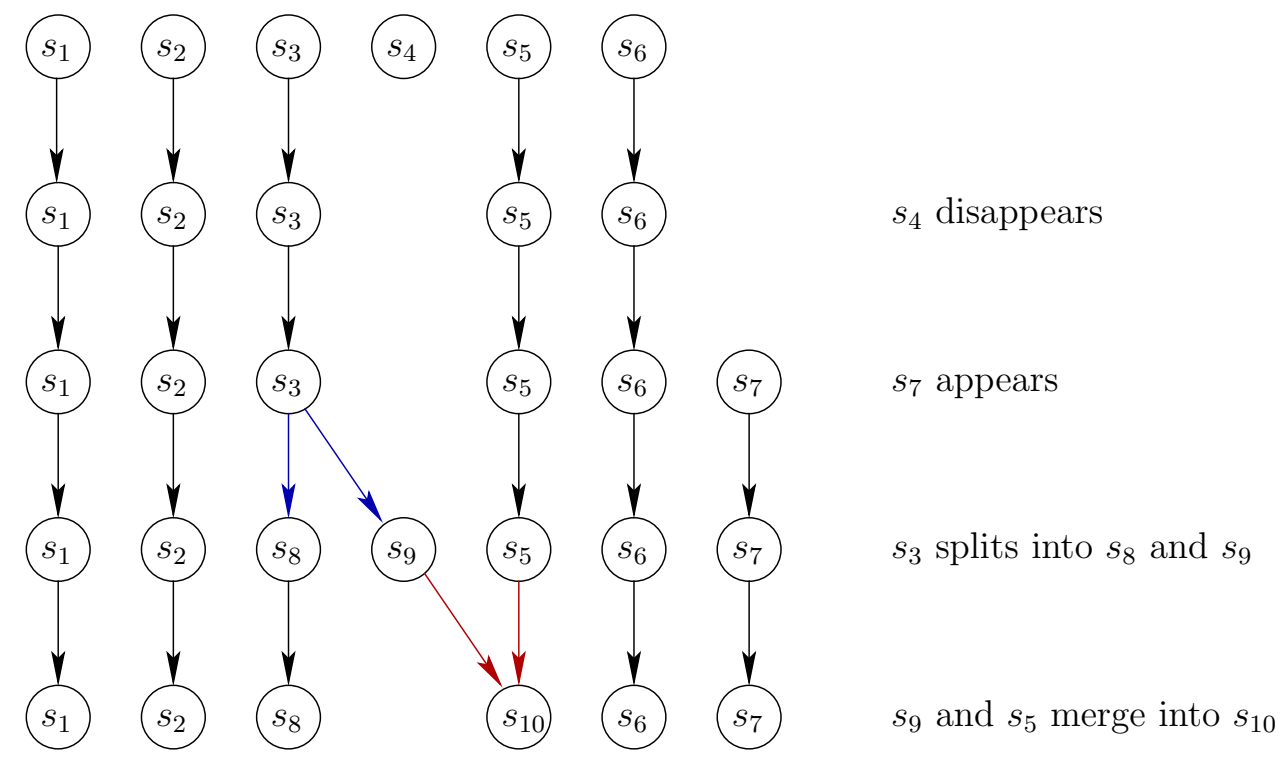

Figure 37: This example shows five stages, caused by each of the four combinatorial events.

any agents. This is useful, for example, to record the status of a pursuit strategy when solving a visibility-based pursuit-evasion problem [18, 23, 47].

The filter needs only to maintain a single bit per component:

- "0" means that there is definitely no body in $s_{1}$

- "1" means that might be a body in $s_{1}$

The filter will obtain all of the information it needs from the shadow sequence I-state. For each set $S_{k}$ of shadow components, we associate a mapping $b_{k}: S_{k} \rightarrow\{0,1\}$ which assigns a status bit to each shadow component. The initial mapping $b_{1}$ is given. If there might be an evader in a shadow component and we have no idea which one, then a common initial assignment is $b_{1}(s)=1$ for every $s \in S_{1}$.

Now consider the operation of the filter when transitioning from $\sigma_{k}$ to $\sigma_{k+1}$. Assume that $b_{k}$ is already computed, and we have to determine $b_{k+1}$. The only additional information used comes from the split and merge relations from stage $k$ to $k+1$. For any shadow component that appears, we assign $b_{k+1}(s)=0$. For any that disappears, there is no assignment to make. The split and merge relations are utilized to handle the other two event types. If $S\left(s, s^{\prime}, s^{\prime \prime}\right)$, then $b_{k+1}\left(s^{\prime}\right)=b_{k}(s)$ and $b_{k+1}\left(s^{\prime \prime}\right)=b_{k}(s)$. If $M\left(s, s^{\prime}, s^{\prime \prime}\right)$, then $b_{k+1}(s)=0$ if and only if $b_{k}\left(s^{\prime}\right)=0$ and $b_{k}\left(s^{\prime \prime}\right)=0$.

To gain an intuition for this filter, imagine that we are searching for a "lost" moving body which is outside of the field of view of the sensors. We do not know where it might be initially, which motivates assigning a value of 1 to every shadow component. As shadow events occur, we must update the bits so that every component $s \in S_{k+1}$ that might contain a body has $b_{k+1}(s)=1$. Any component for which we are sure a body does not lie obtains the 0 status. If components merge, then we can assign 0 only if both original components are certain not to contain a body.

Filter 17 (Count Bounding Filter)

For this filter, we want to keep track of how many bodies there are in every shadow component. This simply replaces each $b_{k}$ function of Filter 16 with two functions $\ell_{k}: S_{k} \rightarrow C$ and $u_{k}: S_{k} \rightarrow C$ 
in which $C=\mathbb{N} \cup\{0, \infty\}$. The function $\ell_{k}$ is the lower bound on the number of bodies in each shadow component. Likewise, $u_{k}$ is the upper bound. As the names suggest, we require that $\ell(s) \leq u(s)$ for any shadow component $s$.

The first functions $\ell_{1}$ and $u_{1}$ are assumed to be given. If no information is available for a shadow component $s$, we could assign $\ell_{1}(s)=0$ and $u_{1}(s)=\infty$. Now consider the incremental operation of the filter. If a component $s$ appears, it receives $\ell_{k+1}(s)=u_{k+1}(s)=0$. If a component disappears, there is nothing to do. If $S\left(s, s^{\prime}, s^{\prime \prime}\right)$, then $\ell_{k+1}\left(s^{\prime}\right)=0, \ell_{k+1}\left(s^{\prime \prime}\right)=0, \ell_{k+1}\left(s^{\prime}\right)=u_{k}(s)$, and $u_{k+1}\left(s^{\prime \prime}\right)=u_{k}(s)$. If $M\left(s, s^{\prime}, s^{\prime \prime}\right)$, then $\ell_{k+1}\left(s^{\prime \prime}\right)=\ell_{k}(s)+\ell_{k}\left(s^{\prime}\right)$ and $u_{k+1}\left(s^{\prime \prime}\right)=u_{k}(s)+u_{k}\left(s^{\prime}\right)$.

So far the filter seems to lose important information. Let $c, c^{\prime}$, and $c^{\prime \prime}$ be the actual number of bodies in $s, s^{\prime}$, and $s^{\prime \prime}$, respectively. Even though these quantities are unknown, if $S\left(s, s^{\prime}, s^{\prime \prime}\right)$, then we must have $c=c^{\prime}+c^{\prime \prime}$. Likewise, if $M\left(s, s^{\prime}, s^{\prime \prime}\right)$, then $c+c^{\prime}=c^{\prime \prime}$. The filter should keep track of this information as well.

An interesting interpretation can be made. Let $S_{k}^{\prime}$ be the set of all shadow components observed up to stage $k$, even including ones that have disappeared. Let $m$ be the number of elements in $S_{k}^{\prime}$. For each $i$ from 1 to $m$, an unknown integer represents the number of bodies in a particular shadow component. An $m$-dimensional vector $v$ of integers can represent the number of bodies in every component. The set of all possible $v$ is a subset of $\mathbb{Z}^{m}$ (each $\mathbb{Z}$ is the set of all integers). For component of $v$, upper and lower bounds are determined, which means that $v$ is restricted to an axis-aligned rectangular subset of $\mathbb{Z}^{m}$. Furthermore, constraints of the form $c=c^{\prime}+c^{\prime \prime}$ and $c+c^{\prime}=c^{\prime \prime}$ due to splits and merges further restrict the set of possible $v$. Since all constraints are linear, the set of allowable $v$ all lie in a polytope in $\mathbb{R}^{m}$. To ask particular questions about how many bodies are in a particular shadow component, taking all constraints into account, an integer linear program is obtained. Efficient solutions to this particular program are described in [53]

Filter 18 (Team Maintenance Filter)

Filter 17 can be extended easily to the case of partially distinguishable bodies. Suppose that each body belongs to a team. There could be only one team, which means they are all indistinguishable. If each is on a unique team, then they are all fully distinguishable. As an example in between, suppose they are classified as men or women. This yields two teams, and bodies are assumed to be indistinguishable inside of each team.

For this problem, we simply make a "vector version" of Filter 17, with one part for each team. For each shadow component and team, an upper and lower bound is maintained. All filter updates are handled for each team independently [53].

An important complication can be easily considered in the filters above, and it is important to practical implementations. A body may pass in or out of the detection region $V(q)$, in which case we obtain additional information to be used in the filters. In this case, $V(q)$ may be treated as a special component for which the exact count on the number of bodies is known. Consider extending Filter 17. If we observe a body entering from shadow component $s$ into $V(q)$, then we should decrement by one the upper and lower bounds associated with $s$. Likewise, if a body leaves $V(q)$ and enters some shadow component $s$, then we could increment its bounds. Such details are worked out in [53].

\subsubsection{Gap navigation trees}

We now develop a filter that is closely related to the shadow I-space. If a single robot with a gap sensor is placed into a simply connected environment, Model 25 (simple gap), then every gap 
corresponds directly to a shadow component. For example, when the robot is placed as in Figure 35(a), there are 5 gaps and each one corresponds to a shadow component.

Return to the shadow sequence I-state. We can similarly define a gap sequence I-state. The notions of combinatorial events and stages from Section 4.3.2 are used here. Rather than (75), we obtain

$$
G_{k}=\left\{g_{1}, g_{2}, \ldots, g_{n}\right\}
$$

in which $G_{k}$ is the set of gaps that exist over stage $k$, which is an interval of time during which no combinatorial events occur. We start with $G_{1}$, and have transitions from $G_{k}$ to $G_{k+1}$ in the same way as with shadow components: Gaps may disappear, appear, split, or merge. Furthermore, the merge and split relations are used here. This results in a gap sequence I-state $\gamma_{k}$ and gap sequence I-space, $\mathcal{I}_{\text {gseq }}$.

As part of $\gamma_{k}$, we additionally assume that the split and merge relations can be inferred from the observation history. Here we obtain $M\left(g, g^{\prime}, g^{\prime \prime}\right)$ and $S\left(g, g^{\prime}, g^{\prime \prime}\right)$, defined in the same way as for shadows in Section 4.3.2. Note that this may or may not be possible in practice, depending in particular on how the gap sensor is implemented. In other words, to determine $M\left(g, g^{\prime}, g^{\prime \prime}\right)$, there needs to be sufficient information to infer that $g^{\prime \prime}$ was formed precisely as $g$ and $g^{\prime}$ merged.

Filter 19 (Gap Navigation Tree)

We describe a filter over an I-space $\mathcal{I}_{\text {trees }}$ of rooted trees [51]. Each tree captures some critical structure of the environment and is combinatorially equivalent to the notion of a shortest path tree that arises in visibility algorithms [?]. Initially, for $\gamma_{1}$, the I-state $\iota_{0} \in \mathcal{I}_{\text {trees }}$ consists of a root node that is connected to one child node for every gap in $G_{1}$. Every child vertex is labeled with its corresponding gap name.

The construction of the tree will now be described inductively. Assume that a tree $\iota_{k}$ has been computed by the filter. Using the new gaps $G_{k+1}$, a new tree $\iota_{k+1}$ is formed. Assuming that only one event occurs from stage $k$ to $k+1$, it must either be an appear, appear, split, or merge. The filter is defined by describing the "surgery" that performed on $\iota_{k}$ to obtain $\iota_{k+1}$. For an appear event, a new child is added from the root and given the label of the new gap. For a disappear event, the corresponding child is deleted from $\iota_{k}$ to obtain $\iota_{k+1}$.

For a merge event $M\left(g, g^{\prime}, g^{\prime \prime}\right)$, consider the two subtrees corresponding to the two gaps $g$ and $g^{\prime}$. A new child of the root is inserted with label $g^{\prime \prime}$. The subtrees corresponding to $g$ and $g^{\prime}$ are moved from the root and attached to $g^{\prime \prime}$ to indicate that these were merged into $g^{\prime \prime}$. In the case of a split event, the process works in reverse. For $S\left(g, g^{\prime}, g^{\prime \prime}\right)$, if there are already subtrees corresponding to $g^{\prime}$ and $g^{\prime \prime}$, then these are attached as children of the root when $g$ is deleted. If there are no subtrees labeled $g^{\prime}$ and $g^{\prime \prime}$, then new child nodes corresponding to $g^{\prime}$ and $g^{\prime \prime}$ are attached to the root. More details appear in $[37,51]$.

What is actually being recorded by the tree in Filter 19? The critical events are actually caused by generalized inflections and bitangents. Figures 39 and 40 show these cases. If the robot crosses an inflection ray, then an appear or disappear event occurs, depending on which direction it crosses. If the robot crosses a bitangent ray, then a split or merge event occurs, again depending on the direction. It turns out that the inner segments of bitangents are part of a well-known structure called the shortest-path graph (or sometimes called the reduced visibility graph [34]). An example is shown in Figure 38. Consider all pairs $p, p^{\prime}$ of points in a simply connected polygonal environment $E$. For each pair, there is a unique shortest path, which happens to be piecewise linear. Except for the initial and final path segments, all other segments must be either polygon edges or bitangent edges. Every nonsmooth point along the path corresponds to a reflex vertex along $\partial E$ (interior angle greater than $\pi$ ). 


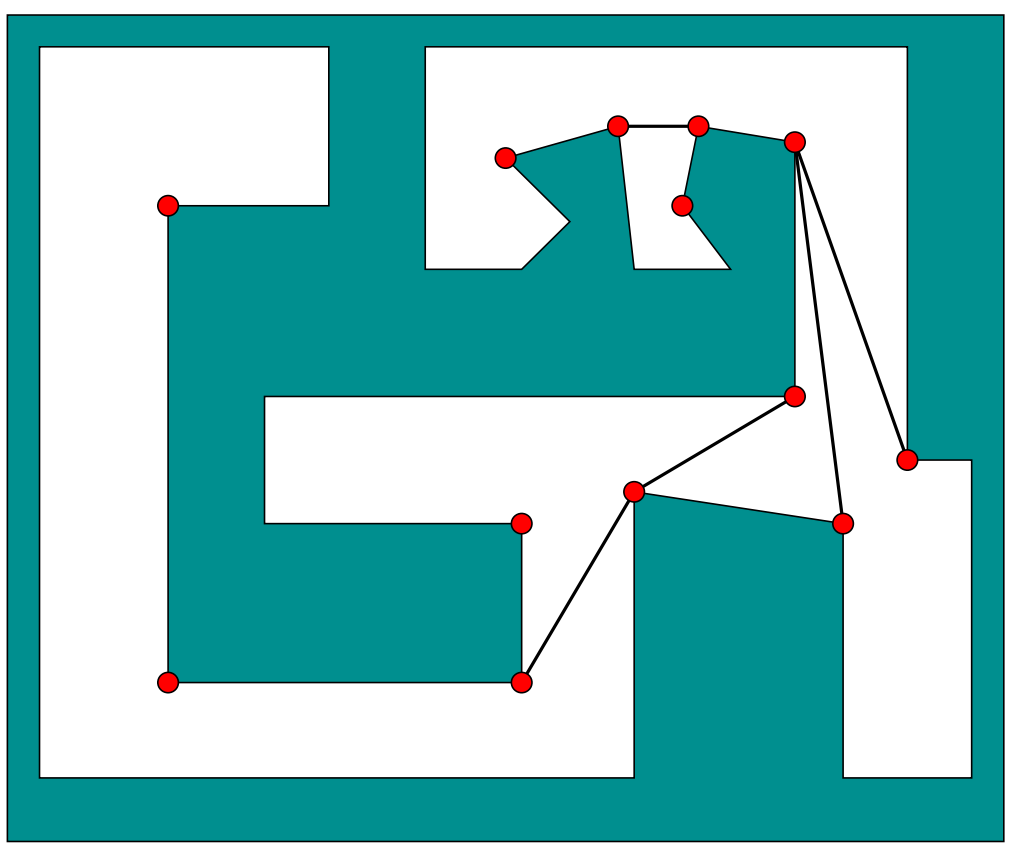

Figure 38: The shortest-path graph. It is somtimes referred to as a (reduced) visibility graph [34].

If the robot explores enough of the environment, it is shown in [51] that the tree produced by Filter 19 encodes a portion of the shortest-path graph that is sufficient for optimal navigation to any place in $E$ from the current robot location. This is depicted in Figure 41. Distance-optimal navigation can be performed using this filter, and is briefly described in Section 5.1.3.

\subsection{Grid-based exploration and mapping}

For the final examples of filters, consider a grid-based physical state space. Although this does not correspond to the way we ordinarily view the physical world, it is convenient for modeling purposes and has been used in many previous works. See Figure 42(a). There is a single robot, with position coordinates described by a pair $(i, j)$ of integers. There are only four possible orientations (such

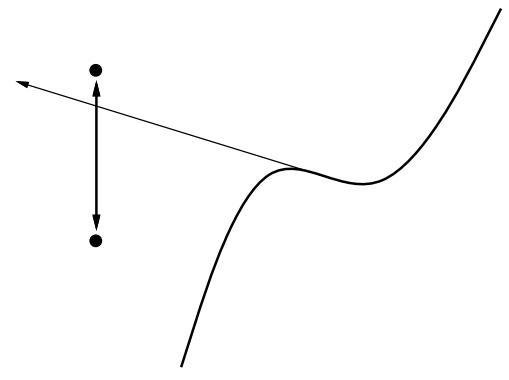

(a)

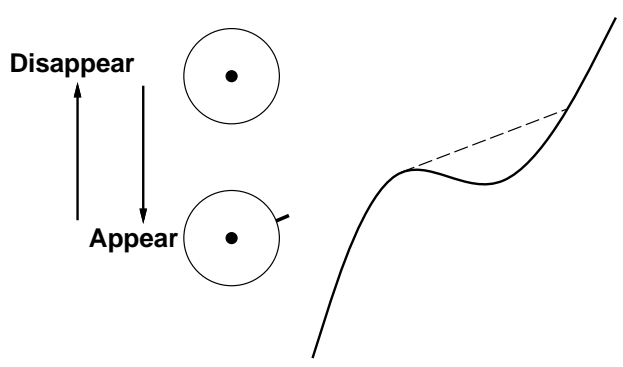

(b)

Figure 39: (a) The robot crosses a ray that extends from an inflectional tangent. (b) A gap appears or disappears from the gap sensor, depending on the direction. 


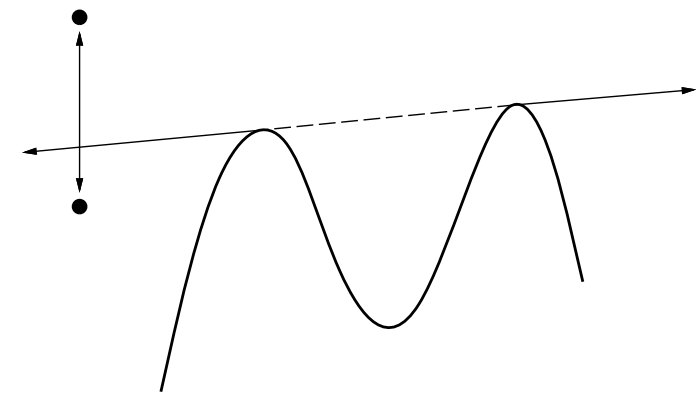

(a)

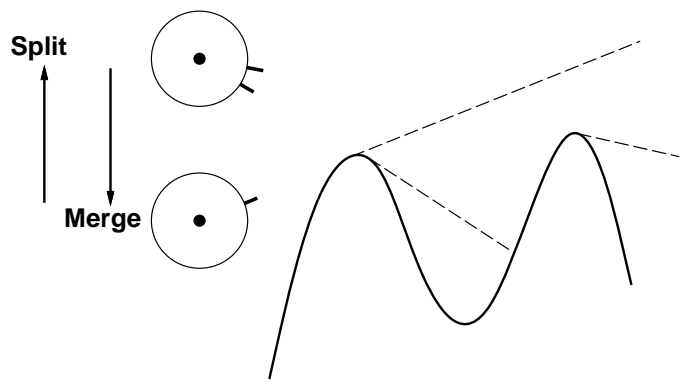

(b)

Figure 40: (a) The robot crosses a ray that extends from a bitangent. (b) Gaps split or merge, depending on the direction.

as north, east, west, south). Let $\mathbb{Z} \times \mathbb{Z}=\mathbb{Z}^{2}$, in which $\mathbb{Z}$ is the set of all integers. At every $(i, j)$, suppose a tile is placed which may be either "white" or "black". The robot may occupy any white tile at any time; however, it is unable to occupy a black tile.

Recall the continuous-space models from Section 3.1.1. For a known map, the state space was $X \subset \mathbb{R}^{2} \times S^{1}$. For the grid-based counterpart to this model, $\mathbb{R}^{2}$ is replaced by $\mathbb{Z}^{2}$ and $S^{1}$ is replaced by the set $D$ of four directions. An environment, or map, $E$ is just a connected set of white tiles. This can be expressed as a set of $(i, j)$ pairs. If $E$ is known, then $X=E \times D$. This would be appropriate for a discrete robot localization problem. However, it is more interesting to consider a simultaneous localization and mapping (SLAM) problem, in which neither the robot configuration nor the map $E$ are known. In this case, let $\mathcal{E}$ denote the set of possible environments, resulting in

$$
X \subset \mathbb{Z} \times \mathbb{Z} \times D \times \mathcal{E}
$$

More precisely, $X$ is the set of all $(i, j, d, E)$ for which $(i, j) \in E, d \in D$, and $E \in \mathcal{E}$.

Now we create a motion model. Only two actions are needed: 1$)$ move FORWARD in the direction the robot is facing, and 2) ROTATE the robot 90 degrees counterclockwise. If the robot is facing a black tile and FORWARD is applied, then a sensor reports that it is blocked and the robot does not move.

Suppose that the robot is initially placed on a white tile, in an unknown environment, with an unknown orientation. What kinds of filters can be made? Consider once again implement the nondeterministic filter (Figure 9). The filter I-space is $\mathcal{I}=\operatorname{pow}(\mathbb{Z} \times \mathbb{Z} \times D \times \mathcal{E})$. What are the possible states consistent the history I-state?

Filter 20 (Naive Map List)

The nondeterministic filter computes $X_{k+1}\left(\eta_{k+1}\right) \subset X$ from $X_{k}\left(\eta_{k}\right)$ after each action and observation. Without any insights, it appears we must enumerate all possible maps $E \in \mathcal{E}$ that are consistent with the history I-state, and for each one, enumerate all possible $(i, j) \in \mathbb{Z} \times \mathbb{Z}$ and orientations $d \in D$. Since $X_{k}\left(\eta_{k}\right)$ is infinite, it is impossible to directly represent it as a set of sets of tiles. One possibility is to declare in the model that all environments $E$ must fit into a bounded region. For example, suppose it is known that if $|i|>1000$ or $|j|>1000$, then the tile must be black. In this case, we can apply (60) to compute the effect of an action, and (61) to take into account the sensor reading. Obviously, this is horribly inefficient. 

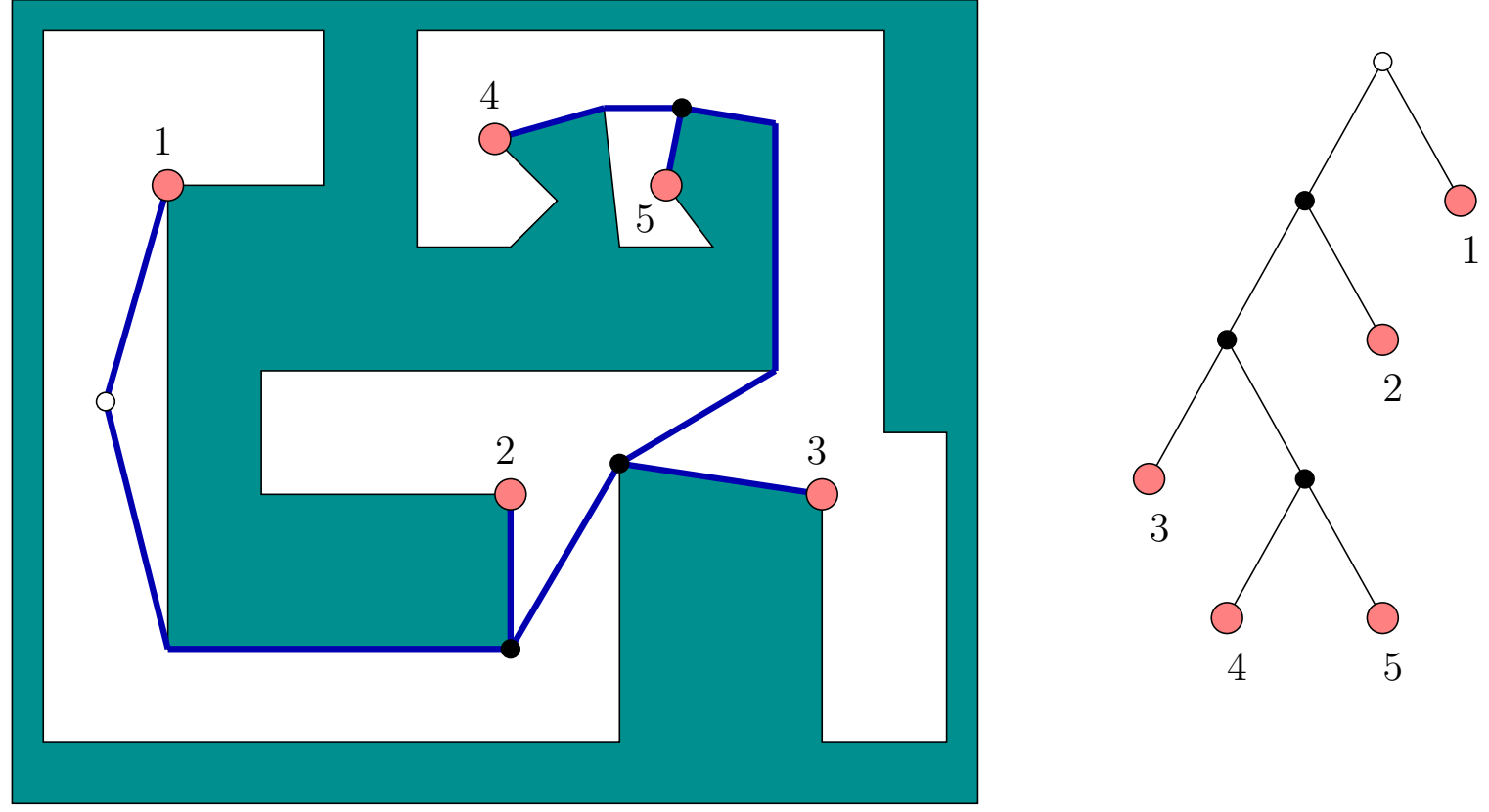

Figure 41: The gap navigation tree captures the structure of the shortest paths to the current robot location. The robot position is shown on the left. The tree on the right characterizes precisely how the shortest to the robot location are structured.

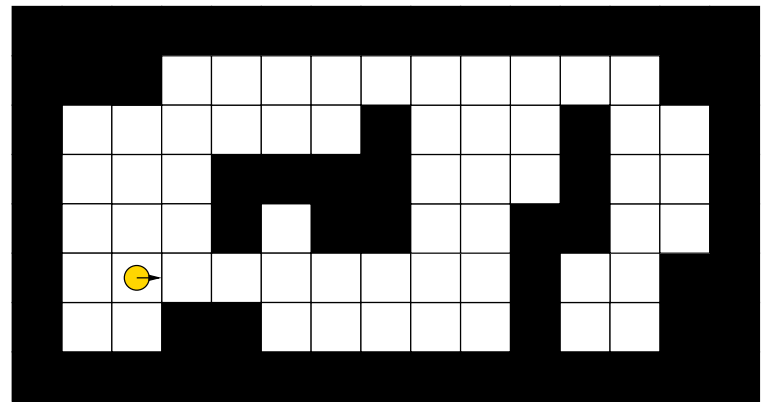

(a)

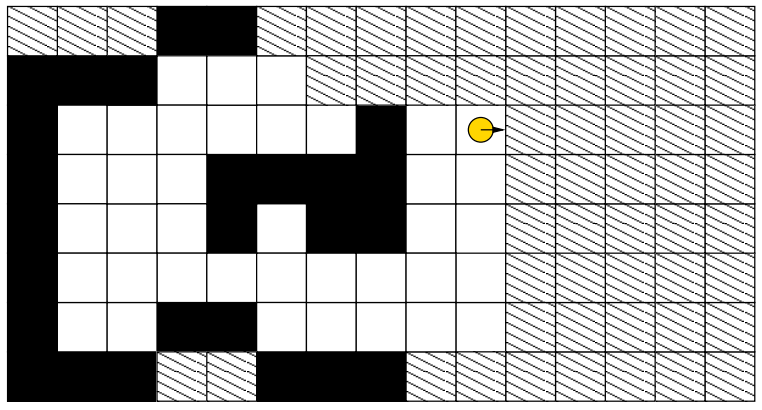

(b)

Figure 42: (a) A discrete grid problem is made in which a robot is placed into a bounded, unknown environment. (b) An encoding of a partial map, obtained from some exploration. The hatched lines represent unknown tiles (neither white nor black). 
Filter 21 (Keeping a Partial Map)

A much improved filter can be made by compactly representing the set of possible maps, as shown in Figure 42(b). Rather than being forced to label every $(i, j) \in \mathbb{Z} \times \mathbb{Z}$ as "black" or "white", we can assign a third label, "unknown". Initially, the tile that contains the robot is "white" and all others are "unknown". As the robot is blocked by walls, some tiles become labeled "black". The result is a partial map that has a finite number of "white" and "black" tiles, with all other tiles being labeled "unknown". An I-state can be described as two finite sets $W$ (white tiles) and $B$ (black tiles), which are disjoint subsets of $\mathbb{Z} \times \mathbb{Z}$. Any tiles not included in $W$ or $B$ are assumed to be "unknown".

A further compression has been assumed. Since it is impossible to determine the precise initial position and orientation, it seems harmless to arbitrarily invent some initial values. If the map were given, then possible positions and orientations would be constrained, but without the map, there is no way to know. This should match your intuition. If I place you into Kentucky and give you a map, you can describe your location in terms of the map's coordinates. However, if you are dropped into Kentucky without a map, then you might as well declare your starting position to be the origin of the personal map that you will construct.

Therefore, the robot is declared initially to be at tile $(0,0)$ and facing east. All other $i$ and $j$ coordinates are derived from the action history, which is essentially discrete odometry. The partial map shown in 42(b) actually represents an equivalence class of partial maps that can be superimposed using a discrete translation and rotation. All of this has been nicely compressed into a single representation.

\section{Discussion}

In this article, Section 2 introduced a wide range of physical sensors. Section 3 then developed the notion of virtual sensors as mappings $h$ from a predetermined physical state space $X$ into an observation space $Y$. This allowed sensors to be modeled in an implementation-independent way. We then defined many categories of sensors which are compared in terms of $h$ 's preimages. These models were also extended in several ways, including mappings over state-time space $Z$, state trajectory space, $\tilde{X}$, and disturbances that are either nondeterministic or probabilistic. Section 4 then provided methods for aggregating observations made from spatially or temporally distributed sensors. The main idea is to recall is that efficient filters can often be designed through a careful choice of models, rather than incurring the full cost of generic probabilistic or nondeterministic filters.

The remainder of this section concludes the paper by giving ideas for uses of simple sensors and filters, indicating some topics for further reading, and presenting open challenges and problems. There are many exciting directions for future research and timely opportunities to impact the development of robust, efficient, low-cost systems that mix sensors, actuators, and computation.

\subsection{From Filtering to Planning and Control}

Section 4 covered the passive problem of gathering data from sensors and recorded actions, and then making inferences. Filters can be used to estimate the state, count the number of bodies, and so on, but they do not actively manipulate the state. In Section 5.1, we briefly explain how to connect the outputs of filters to the set of actions so that actions are chosen based on the filter I-state. The result may be called a plan, control law, policy, strategy, and so on. The process of 
funding such a solution may be called planning or control. Below, we choose the terms plans and planning.

We view all planning problems as a search or optimization in an I-space, rather than the state space (unless the I-space happens to coincide with the state space). When there is substantial uncertainty due to sensors, planning occurs over an appropriate I-space. The challenging task is to design the system and resulting I-space so that task-solving plans can be efficiently computed and successfully executed. Ideally, we would like to accomplish tasks without having to fully reconstruct or estimate the full external state.

\subsubsection{Plans and Execution}

Let $\mathcal{I}$ be any I-space. Suppose that a state transition model from Section 4.2.4 is available that includes an action space $U$. Furthermore, suppose that a filter $\phi$ has been defined of the form (57). This produces a new I-state $\iota_{k} \in \mathcal{I}$ from the previous I-state $\iota_{k-1} \in \mathcal{I}$, previous action $u_{k-1} \in U$, and new observation $y_{k} \in Y$.

Generally, the planning problem is to chose each $u_{k}$ so that some predetermined goal is achieved. Let $G \subset \mathcal{I}$ be called a goal region in the I-space. This should be correspond to the achievement of a goal with respect to the physical world; if $G$ cannot be defined to ensure this, then $\mathcal{I}$ must be redesigned. Starting from an initial I-state $\iota_{0} \in \mathcal{I}$, what sequence of actions $u_{1}, u_{2}, \ldots$, will lead to some future I-state $\iota_{k} \in G$ ? Since future observations are usually unpredictable, it may be impossible to specify the appropriate action sequence in advance. Therefore, a more complete way to specify the action selections is to define a plan:

$$
\pi: \mathcal{I} \rightarrow U
$$

In this case, an action is determined from every I-state. During execution of the plan, the filter is executed, I-states are generated, and actions get automatically applied using $\pi$.

Using a filter $\phi$, the execution of a plan can be incorporated as

$$
\iota_{k}=\phi\left(\iota_{k-1}, y_{k}, \pi\left(\iota_{k-1}\right)\right),
$$

which makes the filter no longer appear to depend on actions. The filter runs autonomously as the observations appear.

The main challenge is to construct a plan that achieves the desired goals. This paper provides only a starting point for addressing this. The main issues that arise repeatedly in planning are:

- Predictability: Are the effects of actions predictable in the I-space? If not, then plans may be considered in which actions depends on future, unpredictable I-states. Otherwise, execution may look like a predictable path in the I-space.

- Reachability: Is the goal region even reachable from the initial I-state? In other words, do there even exist actions that will take us to the goal? Also, based on the I-space, does there exist a plan that can reach the goal? If there is unpredictability, then we might additionally require that the goal is guaranteed to be reachable, over all possible disturbances.

- Optimality: If there are many plausible alternative plans, then what cost criteria should be formulated, and which plans are optimal with respect to them? Do optimal plans even exist?

- Computability: Given a description of the problem, can an algorithm be determined that automatically computes a useful plan? In many cases, a plan is designed by a clever human; 
however, automated planning is desirable in many circumstances. If a plan is theoretically computable, there are still practical issues such as algorithm complexity (running time and space) and implementation difficulty.

\subsubsection{Important Generic Examples}

Several examples are given here in which plans are described over various I-spaces that appeared in Section 4. They are widely used in robotics, planning, and control theory. Their description here, however, they may look unusual because we based all of them on I-space concepts.

Example 2 (State Feedback Plans)

Suppose we have a filter that produces a reliable estimate of $x_{k}$ using $\eta_{k}$. Assume the filter fits the general form (57), in which the I-space is $\mathcal{I}=X$ and $\iota_{k}$ is the estimate of $x_{k}$. In this case, a plan as expressed in (78) becomes $\pi: X \rightarrow U$.

Once the filter is running, there is no need to worry in the planning stage about uncertainty with regard to the current state. All sensing uncertainty is the problem of the filter. This is a standard approach throughout control theory and robotics: Produce a good state-estimating filter and then produce a plan or policy that uses state feedback. This enables the two issues of sensing and planning to be decoupled. Although a useful approach in many settings, we are most interested in this paper in ways to analyze both together, leading to simpler I-spaces, filters, and planning problems.

Example 3 (Open-Loop Plans)

In this case, we use Filter 6, which simply counts the number of stages. Recall the simple update equation (50) and I-space $\mathcal{I}=\mathbb{N}$. A plan is expressed as $\pi: \mathbb{N} \rightarrow U$. This can be interpreted as a specifying a sequence of actions:

$$
\pi=\left(u_{1}, u_{2}, u_{3}, \ldots\right)
$$

Such plans are often called open loop because no significant sensor observations are being utilized during execution. However, be careful, because some implicit time information is certainly being used: It is known that $u_{3}$ is being applied later than $u_{2}$, for example.

In (80), the actions appear to execute forever. In practice, the plan may terminate after a finite number of stages. See Chapter 2 of [37] for discussions of termination issues.

Example 4 (Sensor-Feedback Plans)

Now suppose that Filter 5 is applied, which produces only the most recent sensor observation $y_{k}$. In this case, a plan becomes $\pi: Y \rightarrow X$. It is wonderfully simple if such a "reactive" plan can solve a useful task. For most tasks, however, some history of observations is needed.

Example 5 (Plans Over History I-Space)

Recall Filter 8, which simply reports the complete history of all observations and actions obtained so far. In this case, a plan is $\pi: \mathcal{I}_{\text {hist }} \rightarrow U$, which appears to be the most powerful plan possible. Every action depends on all possible information that can be utilized. There are several drawbacks, however. Since $\mathcal{I}_{\text {hist }}$ is large, it maybe be difficult or impossible to even represent an interesting plan. Furthermore, it may be hard to determine that the plan is in fact achieving a goal. Expressing the goal in this I-space may also be impractical because there is no direct connection to the state space. 


\subsubsection{Problem-Specific Examples}

The examples of Section 5.1.2 used generic I-spaces that do not necessarily take into account problem-specific information to reduce the overall complexity of the planning problem. Using the concepts of this paper, we encourage the following overall process:

1. Consider a system, which includes the environment, bodies, and sensors, and is designed for some task or tasks.

2. Define the models, which provide the state space $X$, the sensor mapping $h$, and the state transition function $f$.

3. Select an I-space $\mathcal{I}$ for which a filter $\phi$ can be practically computed.

4. Take the desired goal, expressed over $X$, and convert it into an expression over $\mathcal{I}$.

5. Compute a plan $\pi$ over $\mathcal{I}$ that achieves the task in terms of $\mathcal{I}$.

Ideally, these steps should all be taken into account together; otherwise, a poor choice in an earlier step could lead to artificially high complexity in later steps. Worse yet, a feasible solution might be lost. Consider how Steps 4 and 5 may fail. Suppose that in Step 3, a simple I-space is designed so that each I-state is straightforward and efficient to compute. If we are not careful, then Step 4 could fail because it might be impossible to determine whether particular I-states achieve the goal. For example, Filter 6 simply keeps track of the current stage number. In most settings, this provides no relevant information about what has been achieved in the state space. Suppose that Step 4 is successful, and consider what could happen in Step 5. A nice filter could be designed with an easily expressed goal in $\mathcal{I}$; however, there might exist no plans that can achieve it. It could be the case that the problem is impossible to solve in the physical world under any circumstances, but a more common problem is that sufficient progress cannot be made in $\mathcal{I}$. This could mean, for example, that when actions are applied as in (79), the desired I-states are not even reachable.

Just to give the idea, we consider a couple of examples that utilize the filters of Section 4.

Example 6 (Gap Navigation Trees)

Suppose that we would like to fully explore a continuous, planar environment. The map, together with position and orientation, is replaced in the current setting by a tree.

We start with Filter 19 and additionally introduce a motion model. The idea is that the robot can "chase" a gap, which means that an action $u$ can be given so that the robot moves in the direction of the gap until a critical event occurs: The gap will either disappear or split [37, 51].

The first task is to make a plan that explores the whole environment. Recall the "unknown" labels from Example 21. The analogous issue here is that each leaf of the tree might contain a part of the environment that has not yet been explored. This means that if we chase that gap, we do not know whether it will disappear or split. We therefore augment Filter 19 so that it records a single bit of information for each leaf node. If we are certain that the gap would disappear, then we label the corresponding leaf node as "primitive". The search strategy is to chase any ancestor of a nonprimitive leaf node, causing a sequence of splits, and terminating with a critical event for the leaf node. If it splits, then a descendant is chased. If it disappears, then a new non-primitive leaf node is selected and the plan continues. It is shown in [51] that in a simply connected environment, this plan always terminates with all leaves labeled as "primitive". At this point, the entire environment has been explored.

Once the entire environment has been explored, the tree can also be used for optimally moving the robot between locations. Since there are no coordinates in which to express the goals, the sensing 
model and filter are slightly augmented so that an object can be placed in $E$ and is detected when it is visible from the robot. As the object disappears from view, it is simply recorded in the tree as if it were a disappearing gap. To return to the object optimally in terms of the shortest possible Euclidean distance traveled, the plan chases every ancestor of the object [51].

The process above was followed by selecting a specific I-space in which the filter could be efficiently computed, the task could be specified, and a successful plan was found.

Example 7 (Searching a Grid)

Recall Filter 21. Suppose now that we want to use the filter to move the robot so that every tile in $E$ is guaranteed to be visited. This strategy could be used to find an lost "treasure", which has been placed on an unknown tile.

Here is a simple plan that uses this filter. For any "unknown" tile that is adjacent to a "white" tile, we attempt to move the robot onto it to determine how to label it. This process repeats until no more "unknown" tiles are reachable, which implies that the environment has been completely explored. Several algorithm details are missing here, but you should be able to implement it without trouble.

The method works; however, a far more interesting filter and plan are given in [5] by carefully using the task to reduce filter complexity. Their filter maintains I-states that use only logarithmic memory in terms of the number of tiles, whereas recording the entire map would use linear memory. They show that with very little space, not nearly enough to build a map, the environment can nevertheless be systematically searched. For this case, the I-state keeps track of only one coordinate (for example, in the north-south direction) and the orientation, expressed with two bits. An entire plan is defined in [5] that is guaranteed to visit all white tiles using only this information (the method is also summarized in Section 12.3.1 of [37]).

\section{$5.2 \quad$ Related subjects}

The topics covered in this paper touch a wide variety of disciplines and research fields. The sensor-centric perspective introduced here is quite unique; concepts such as sensor lattices and combinatorial sensors have not been introduced before. To fully understand and apply the ideas to problems of interest, it is helpful to understand how they relate to existing fields. Therefore, some connections and tips for further reading are presented here. This is not meant as a brief survey of "everything"; instead, it is a list of pointers to related topics as seen from this unusual perspective. For further reading on physical sensors themselves, see [17, 52].

Mobile robotics is the main inspiration for the concepts presented here, particularly the problems of mobile robot localization, simultaneous localization and mapping (SLAM), navigation, pursuitevasion, target tracking, and searching. Most approaches require learning as much as possible about the model and the robot configuration while proceeding. For example, nearly all SLAM approaches construct complete, precise geometric maps of the environment and maintain the robot configuration at all times $[13,49]$. The main source of uncertainty handled is sensor noise/disturbances, rather than the preimages of Section 3.2.2. Probabilistic modeling and sampling-based algorithms are employed to compute the I-states of Bayesian filters (Filter 10). There are recent efforts to develop reduced-complexity probabilistic I-spaces (called belief spaces) for the purpose of planning specific tasks $[25,32,44]$. Just as the Bayesian filter I-space of all posterior pdfs collapsed nicely into the Kalman filter I-space of Guassians, attempts are made to restrict or approximate the space of pdfs. Within robotics, preimages of sensor mappings have been considered in earlier works 
$[11,10]$. Furthermore, preimages of the state transition mapping has also been explored, which have the interesting property of reducing uncertainty as preimages become larger [15, 16, 20, 35, 38], which is contrary to sensor preimages. In addition to the probabilistic I-spaces mentioned above, nondeterministic I-spaces have appeared in robotics work in [14, 23].

Robotics itself is closely connected to the emerging field of cyber-physical systems (CPS), which covers engineering systems that combine sensing, actuation (or control), and computation. The CPS field is a culmination of research from three earlier disciplines: 1) hybrid systems, which involves the design of control laws for state spaces that have both discrete and continuous components; 2) sensor networks, which studies filtering and networking issues for a distributed collection of sensors that wirelessly transmit information; 3) real-time and embedded systems, which develops specialpurpose computer systems placed into the physical world, typically involving sensor measurements. With regard to hybrid systems, this paper certainly has mixed observation spaces with continuous state spaces; however, the discrete vs. continuous distinction is not as critical as external physical state vs. internal information state. Sensor networks typically involve stationary nodes; however, the extension to actuated sensor networks is essentially a multiple mobile robot system. In addition to filtering, however, concerns such as overall power consumption and networking efficiency are typically emphasized. With regard to real-time and embedded systems, the paper may indicate new types of filters that could be developed across space and time. With regard to CPS in general, there is currently great interest in developing mathematical foundations. Since sensors must extract information from the physical world, it seems that sensor mappings, preimages, sensor lattices, filters, and information spaces must be part of such a foundation.

Also related to robotics are the fields of computer vision, compressed sensing, sensor fusion, and machine learning. Computer vision and image processing focus on cameras, which are the most widely utilized sensing sources. Vision techniques may be helpful for the physical implementation of many virtual sensor models discussed in Section 3.2. Identifying or counting people, for example, is a common vision task. At the intersection of robotics and vision lies visual servoing, which performs robot control in the observation space of a camera [27]. Compressed sensing is a field that has recently exploded with interest [12] by showing that extremely low resolution devices (for example, a single-pixel camera) provide sufficient information for reconstruction. This implies enormous preimages in the sensor mapping, which fits nicely with the minimalist philosophy in this paper. The approaches can be considered as a form of random-projection-based dimensionality reduction, enabled by the Johnson-Lindenstrauss Lemma. Sensor fusion has for decades been concerned with the integration of sensor data from multiple sources, mainly for the purpose of inference [24]. This paper takes a preimage-based approach to this integration, which is most evident in the general triangulation principle of Section 4.1. Finally, machine learning provides powerful techniques for discovering statistical relationships between observed data. This can be useful for developing probabilistic sensor mappings and motion models. At one extreme, it is sometimes possible to achieve desired outputs of a system by gathering input-output observations without directly defining an external physical state space. This article, on the other hand, assumes that powerful behaviors of the physical world, such as continuity of motion, the impassibility of obstacles, rigid body mechanics, and so on, should be exploited in the filter design process.

The similarity of the names information spaces and information theory suggests a connection between them, but this is more of a name coincidence. Information theory [9] is part of communication theory in which the model looks much like a telegraph: A medium or channel is used to send messages from one end to another. The central question is: How much information can be pushed through the channel within a given time? This naturally led to a precise characterization of information itself in terms of bit complexity. The term information space comes from sequential (or dynamic) game theory, in which players in a game might not have access to the state after 
decisions have been made. Imagine, for example, the classical game of Battleship. How can possible ship locations be encoded during play? In 1944, Von Neumann and Morgenstern refered to a player's state of "incomplete information" which they shortened to state of information [?]. Kuhn later referred to information sets [30], and the idea was extended further to information spaces in stochastic control $[3,31]$ and dynamic game theory [2]. Rather than talking about a communication channel, as in information theory, information spaces in this article mainly arise due to the mapping between the physical world and the internal computation states. This mapping may or may not be viewed as a communication channel, and comminucation issues are independent of the important preimage structure due to the mapping itself.

Returning to Figure 1, the connections to the classical theory of computation and algorithms should be more clear. Rather than imagining an automaton interacting with a tape, we connect the automaton (or a whole Turing machine) to the physical world and develop algorithms that account for the preimages due to the sensor mapping. In this way, the "state" has both external and internal components. The external components are most often continuous. The external space may be enormous, but it does not have to be encoded in the usual way for computation. The filters of Section 4 may be considered as algorithms in the traditional sense, but it important to keep in mind the sensor mappings that produce the information. The overall complexity of the system depends on this. The "input" for these algorithms is essentially the physical world. When the physical world is taken into account, alternative notions of decidability and complexity should follow. In some of our own work we have taken early steps in this direction by comparing sensors in the sensor lattice in Section 3.3 and by developing a general approach to comparing robotic systems based on their reachability characteristics in I-spaces [43].

Section 5.1 briefly expressed now this article is related to planning and control theory. Much of the state space concepts, models, and notation in this article follow from control theory. For example, $y=h(x)$ is the standard notation used to express an output mapping. In its classical form, however, $y$ is usually not discrete and computation states are not considered. For a more complete introduction to planning that fits with the notation of this article, see [37]. Other helpful references include $[8,34]$. Planning and control are also closely related to verification, which involves showing that there does not exist a solution policy, rather than finding one. In both the cases of planning and control, it has almost always been assumed that the external, physical state needs to be fully recovered through powerful sensors and filters so that state feedback can essentially be applied. This decouples the problem of "handling uncertainty" from the driving the system to its goal. This is in sharp contrast to the main theme in this article, which advocates leaving the external state ambiguous while nevertheless assuring that a task is achieved.

\subsection{Future Challenges: Help!}

Numerous open and interesting research challenges remain, both in the design of particular systems and in establishing general mathematical foundations. When applying the ideas of this article to a particular system, it is important to consider the task carefully so that the virtual sensor model provides sufficient information without including irrelevant data. For example, it is advantageous early on if it is recognized that a binary detection sensor is sufficient, rather than a detailed camera image. Following in this way, it may be possible to design spatial and temporal filters that have substantially reduced complexity in terms of computation time and space. This often involves finding an I-space that contains enough information to solve the problem and for which updates can be efficiently computed when new data are available. For active tasks, this may consequently simplify the planning problem, as described in Section 5.1.

In terms of general, basic research, this paper has set up terminology and models and then barely 
scratched the surface on interesting questions that can be formulated and solved. The formulation itself, mainly covered in Section 3, should be extended in many ways. The families of virtual sensors, such depth sensors, relational sensors, and gap sensors are far from complete. Both new sensor mappings should be developed within the families and new families themselves will undoubtedly emerge as different settings and tasks are considered. The additional complications of Section 3.4 require the most development. What virtual sensor models and families are appropriate for nondeterministic and probabilistic disturbances? Can interesting virtual sensor models and families be constructed over state-time space $Z$ ? This is undoubtedly important for distributed systems in which time synchronization is difficult or impossible. At the experimental end, there are opportunities to design physical implementations of the proposed virtual sensors. What virtual sensor models allow low-cost, low-energy, and reliable measurements? Some virtual sensors may appear ideal for solving tasks, but may be impractical. It is therefore crucial to study implementability. Even in cases where implementations exist, there is room to find better alternatives for the same virtual sensor model.

Moving on to Section 4, both spatial and temporal filters need much more development. For the spatial case, what other well-known triangulation methods can be expressed as the intersection of preimages? Can novel spatial triangulation methods be designed for a distributed set of sensors? What is the best way to handle disturbances, either in the nondeterministic or probabilistic setting. Robustness can be achieved by using more sensors, but how can the result of their combination be expressed? Using the state-time space $Z$, what triangulation methods can be developed as the intersection of preimages? Is it possible to cleanly express Global Position System (GPS) techniques as the intersection of $3 \mathrm{D}$ preimages in $Z$ ?

For temporal filtering, what is the key to collapsing the I-space down to simpler forms? The most general spaces $\mathcal{I}_{\text {prob }}$ (probabilistic) and $\mathcal{I}_{\text {ndet }}$ (nondeterministic) are often too difficult to work with directly. A wise choice of virtual sensor model is one important step. The next critical step is the choice of motion model. In the classical case of the Kalman filter, the key was the combination of a linear motion model, linear sensing model, and Gaussian noise (which has closure under linearity). The combinatorial filters of Section 4.3 were enabled by the simple assumption that state trajectories are continuous in the configuration space. A more detailed and precise motion model might intuitively seem advantageous, but it could unnecessarily complicate the filter. The general problem of computing the effect of an action is equivalent to calculating reachable sets of a control system. For nonlinear systems, the best known only algorithms are computationally costly and produce only approximate reachable sets. Therefore, enormous advantages come in the filter design by simplifying the motion model. Many more efficient combinatorial filters based on continuity of motion should be possible, working in a manner similar to Filters 12 to 18. There is also the open problem of finding other motion properties (instead of simple continuity) that might lead to simpler filters. Another direction is carefully studying the task to dramatically reduce complexity, as in Filter 15, the two-bit filter. Finally, can distributed filtering methods be designed that involve spatial triangulation combined with temporal updates? Perhaps multiple, distributed I-states are obtained.

Another interesting direction for future research is the transition from filtering to planning. Historically, planning has started with complete, idealized models and then uncertainty concerns were gradually inserted. By starting with sensor-centric considerations as in this paper, it should be possible to design more effective planning algorithms at the outset, rather than incorporating sensing uncertainty as an afterthought. Earlier examples of this approach in the literature include bug algorithms [28, 29, 36, 40, 39, 48] and sensorless manipulation [16, 21, 41]. Given a planning task and a filter, what further reduction of the I-space is possible? Can a successful plan be found that requires only a coarse partition of the filter I-space? This amounts to preimages in $\mathcal{I}$ from a 
plan $\pi: \mathcal{I} \rightarrow U$, considered as if it were a sensor mapping. Paralleling the development of motion planning methods (see Chapters 5 and 6 of [37]), it should also be possible to develop both combinatorial and sampling-based planning methods for I-spaces. There are some examples for particular problems. Recent sampling-based methods explore probabilistic I-spaces in [25, 32, 44]. Combinatorial approaches to pursuit-evasion have led to exact nondeterministic I-space exploration [18, 23]. However, these are not as general as the methods that work well for motion planning. Can general solutions be developed for I-spaces? Would combinatorial algorithms provide exact solutions for a broad class of problems? Similarly, would sampling-based algorithms provide practical solutions to a wide array of problems?

Finally, the notion of a sensor lattice has not been considered before. For this topic, many interesting challenges remain. Many more virtual sensor models can be arranged in the lattice, leading to related chains, trees, or directed acyclic graphs of sensors. The computational tradeoffs as one virtual sensor is replaced with another in a filter are not well understood. The lattice merely indicates that one sensor can be replaced with another, but may require unreasonable computation. If a sensor is replaced in a system, what is the complexity change for the overall system? The complexity of algorithms depends on the input, and it is important to develop algorithmic complexity notions that take into account sensor mappings and their tradeoffs. One final open question is whether sensor lattices or something with comparable power can be developed for sensing models that have nondeterministic or probabilistic uncertainty.

\section{Acknowledgments}

This work is supported in part by NSF grant 0904501 (IIS Robotics), NSF grant 1035345 (CNS Cyberphysical Systems), DARPA SToMP grant HR0011-05-1-0008, and MURI/ONR grant N0001409-1-1052. I am grateful to the helpful feedback provided by Wolfram Burgard and Michael Kaess. 

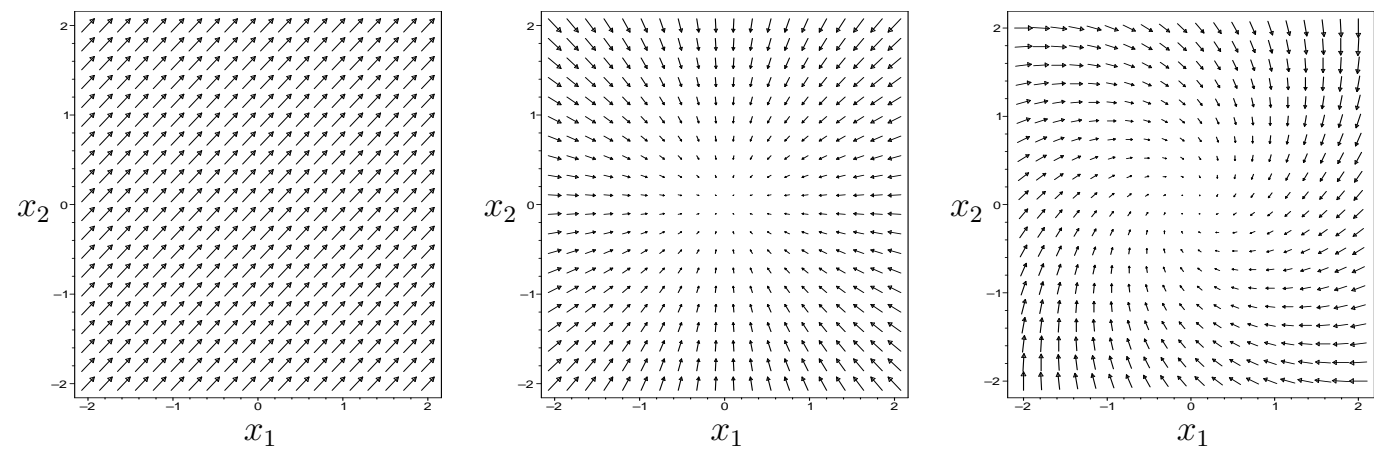

Figure 43: The physical world may contain vector fields on which to base sensor measurements.

\section{A Appendix}

\section{A.1 Models Based on Fields}

\section{A.1.1 Field Definitions}

A field is a function $f: \mathbb{R}^{n} \rightarrow \mathbb{R}^{m}$, in which $n$ is the dimension of the environment $(n=2$ or $n=3)$ and $m$ could be any finite dimension. Usually, $m \leq n$.

As a first example, a map in Section 3.1.1 could equivalently be expressed as a function $f: \mathbb{R}^{2} \rightarrow$ $\{0,1\}$ in which $f\left(q_{x}, q_{y}\right)=1$ if and only if $\left(q_{y}, q_{y}\right) \in E$. This causes a clear division of $\mathbb{R}^{2}$ into an obstacle region and a collision-free region; however, it is sometimes useful to assign intermediate values. Let the map be defined as $f: \mathbb{R}^{2} \rightarrow[0, \infty)$ in which $f\left(q_{x}, q_{y}\right)$ yields an altitude. For an outdoor setting, $f$ could describe a terrain map. In this case, $\mathcal{E}$ is a set of functions in which each $f \in \mathcal{E}$ satisfies some properties, such as a bound on the maximum slope. If there are no other obstacles, then the state space would be $X=S E(2) \times \mathcal{E}$.

Perhaps the most important example is the electromagnetic field generated by a radio transmitter. In a $2 \mathrm{D}$ environment, this is captured by a vector field $f: \mathbb{R}^{2} \rightarrow \mathbb{R}^{2}$; see Figure 43 . Thus, a $2 \mathrm{D}$ vector is produced at every point in $\mathbb{R}^{2}$. A simplified version could be defined as an intensity field, $f: \mathbb{R}^{2} \rightarrow[0, \infty)$, in which the scalar values represent the signal intensity (the magnitudes of the original vectors).

Fields can also be defined with the consideration of other obstacles. For example, waves may propagate through a world that is constrained to a polygonal region.

\section{A.1.2 Field Sensors}

suppose that the world is two-dimensional and a field $f: \mathbb{R}^{2} \rightarrow \mathbb{R}^{2}$ is known. Furthermore, the particular $E$ is known and is simply $E=\mathbb{R}^{2}$. Extensions that remove obstacles from $\mathbb{R}^{2}$ are straightforward. The state space here is simply $X=S E(2)$, which is parametrized as $x=(p, \theta)$.

Model 43 (Direct Field Sensor)

This sensor observes the field vector. The sensor mapping is

$$
h(x)=h(p, \theta)=\left(f_{1}(p), f_{2}(p)\right)
$$

which yields a two-dimensional observation vector. 
Model 44 (Direct Intensity Sensor)

This sensor provides the magnitude of the field vector. For radio signals, this could be achieved using a non-directional signal meter. The sensor mapping is

$$
h(x)=h(p, \theta)=\|f(p)\|,
$$

which yields a nonnegative real intensity value.

Model 45 (Intensity Alarm)

In the spirit of previous sensor models in the section, a binary sensor can be made that indicates when the intensity is above a certain threshold $\epsilon \geq 0$. The sensor mapping is

$$
h(p, \theta)= \begin{cases}1 & \text { if }\|f(p)\| \geq \epsilon \\ 0 & \text { otherwise. }\end{cases}
$$

Model 46 (Transformed Intensity)

In most settings, it is unreasonable to expect to recover the precise magnitude. We might nevertheless have a sensor that returns higher values as the intensity increases. Let $g:[0, \infty) \rightarrow[0, \infty)$ be any strictly monotonically increasing smooth function. The sensor mapping is

$$
h(x)=g(\|f(x)\|) .
$$

If the observations $h(x)$ are linearly proportional to the field intensity, then $g$ is a linear function. In general, $g$ may be nonlinear.

To make the model more interesting, $g$ might not be given. In this case, the set of possible $g$ functions becomes a component of the state space and $g$ becomes part of the state (in other words, $x=(p, \theta, g))$. Such a sensor can still provide useful information. For example, if $y=h(x)$ is increasing over time, then we might know that we are closer to the radio transmitter, even though $g$ is unknown.

\section{Model 47 (Field Vector Observation)}

This sensor directly measures the entire field vector $f(p)$; however, the vector is rotated based on the orientation $\theta$. For example, if the field vector "points" in the direction $3 \pi / 4$ and $\theta=\pi / 4$, then the sensor observes the vector as pointing at $3 \pi / 4-\theta=\pi / 2$. Let $R(\phi)$ be a $2 \times 2$ rotation matrix that induces a rotation by $\phi$. The rotated vector observation is

$$
h_{f v}(x)=R(-\theta) f(p) .
$$

If $f$ is given and $\theta$ is unknown, then it can be determined using $h_{f v}(x)$. Likewise, if $\theta$ is known and $f$ is unknown, then $f(p)$ can be determined from $f(p)=R(\theta) h_{f v}(x)$.

Now consider constructing a magnetic compass. If the field is known, as in the case of the earth's magnetic field, then we can look at the direction of the vector observed using Model 47 and infer the direction $\theta$. The direction with respect to an arbitrary given field is given in the next model. 
Model 48 (Field Direction Observation)

The direction obtained by the observation vector (86). Let $y^{\prime}=h_{f v}(x)$. The sensor mapping is

$$
y=h_{f d o}(x)=\operatorname{atan} 2\left(y_{2}^{\prime}, y_{1}^{\prime}\right)
$$

in which $y \in[0,2 \pi)$ and atan2 is the two-argument arctangent function, common in many programming languages.

Model 49 (Ideal Magnetic Compass)

Suppose it is known that the field vectors are all directed to the north. This means $f(p)=(0,1)$ for all $p \in \mathbb{R}^{2}$. This is, of course, not true of the earth's magnetic field, but we often pretend it is correct. To obtain a compass $y=h(x)=h_{f d o}(x)-\pi / 2$, which adjusts for the angular difference between $\theta=0$ and North, $\theta=\pi / 2$. Under these idealized conditions, we should obtain $y=h(p, \theta)=\theta$.

Model 50 (Magic Compass)

Without even referring to fields, a kind of "magic" compass can be defined as

$$
y=h(x)=h(p, \theta)=\theta .
$$

This is a projection sensor, as defined in Model 5. It somehow (magically) obtains the orientation without using fields.

We can use Model 49 to simulate Model 50 if the perfect field is given. Using perfect calibration, any given field can be used to simulate this compass by simply transforming the angles produced by Model 48. 


\section{References}

[1] V. I. Arnold. Mathematical Methods of Classical Mechanics, 2nd Ed. Springer-Verlag, Berlin, 1989.

[2] T. Başar and G. J. Olsder. Dynamic Noncooperative Game Theory. Academic, London, 1982.

[3] D. P. Bertsekas. Dynamic Programming: Deterministic and Stochastic Models. Prentice-Hall, Englewood Cliffs, NJ, 1987.

[4] D. P. Bertsekas. Dynamic Programming and Optimal Control, Vol. I, 2nd Ed. Athena Scientific, Belmont, MA, 2001.

[5] M. Blum and D. Kozen. On the power of the compass (or, why mazes are easier to search than graphs). In Proceedings Annual Symposium on Foundations of Computer Science, pages 132-142, 1978.

[6] F. Bullo and A. D. Lewis. Geometric Control of Mechanical Systems. Springer-Verlag, Berlin, 2004.

[7] C.-T. Chen. Linear System Theory and Design. Holt, Rinehart, and Winston, New York, 1984 .

[8] H. Choset, K. M. Lynch, S. Hutchinson, G. Kantor, W. Burgard, L. E. Kavraki, and S. Thrun. Principles of Robot Motion: Theory, Algorithms, and Implementations. MIT Press, Cambridge, MA, 2005.

[9] T. M. Cover and J. A. Thomas. Elements of Information Theory. Wiley, New York, 1991.

[10] B. R. Donald. On information invariants in robotics. Artificial Intelligence Journal, 72:217304, 1995.

[11] B. R. Donald and J. Jennings. Sensor interpretation and task-directed planning using perceptual equivalence classes. In Proceedings IEEE International Conference on Robotics $\&$ Automation, pages 190-197, 1991.

[12] D. L. Donoho. Compressed sensing. IEEE Transactions on Information Theory, 52(4):12891306, 2006.

[13] H. Durrant-Whyte and T. Bailey. Simultaneous localization and mapping: Part I. IEEE Robotics and Automation Magazine, 13(2):99-110, 2006.

[14] M. A. Erdmann. Randomization for robot tasks: Using dynamic programming in the space of knowledge states. Algorithmica, 10:248-291, 1993.

[15] M. A. Erdmann. Understanding action and sensing by designing action-based sensors. International Journal of Robotics Research, 14(5):483-509, 1995.

[16] M. A. Erdmann and M. T. Mason. An exploration of sensorless manipulation. IEEE Transactions on Robotics $\&$ Automation, 4(4):369-379, August 1988.

[17] T. Fraichard. Dynamic trajectory planning with dynamic constraints: A 'state-time space' approach. In Proceedings IEEE/RSJ International Conference on Intelligent Robots and Systems, pages 1393-1400, 1993. 
[18] B. Gerkey, S. Thrun, and G. Gordon. Clear the building: Pursuit-evasion with teams of robots. In Proceedings AAAI National Conference on Artificial Intelligence, 2004.

[19] B. Gfeller, M. Mihalak, S. Suri, E. Vicari, and P. Widmayer. Counting targets with mobile sensors in an unknown environment. In ALGOSENSORS, July 2007.

[20] S. K. Ghosh. Visibility Algorithms in the Plane. Cambridge University Press, Cambridge, U.K., 2007.

[21] K. Y. Goldberg. Orienting polygonal parts without sensors. Algorithmica, 10:201-225, 1993.

[22] K. Y. Goldberg and M. T. Mason. Bayesian grasping. In Proceedings IEEE International Conference on Robotics \& Automation, 1990.

[23] L. Guibas. Sensing, tracking, and reasoning with relations. IEEE Signal Processing Magazine, 19(2):73-85, 2002.

[24] L. J. Guibas, J.-C. Latombe, S. M. LaValle, D. Lin, and R. Motwani. Visibility-based pursuitevasion in a polygonal environment. International Journal of Computational Geometry and Applications, 9(5):471-494, 1999.

[25] D. H. Hall and J. Llinas, editors. Handbook of Multisensor Data Fusion. CRC Press, New York, 2001.

[26] R. He, S. Prentice, and N. Roy. Planning in information space for a quadrotor helicopter in a GPS-denied environment. In Proceedings IEEE International Conference on Robotics 8 Automation, 2008.

[27] J. E. Hopcroft and J. D. Ullman. Introduction to Automata Theory, Languages, and Computation. Addison-Wesley, Reading, MA, 1979.

[28] S. A. Hutchinson, G. D. Hager, and P. I. Corke. A tutorial on visual servo control. IEEE Transactions on Robotics \& Automation, 12(5):651-670, October 1996.

[29] I. Kamon and E. Rivlin. Sensory-based motion planning with global proofs. IEEE Transactions on Robotics \& Automation, 13(6):814-822, December 1997.

[30] I. Kamon, E. Rivlin, and E. Rimon. Range-sensor based navigation in three dimensions. In Proceedings IEEE International Conference on Robotics \&5 Automation, 1999.

[31] H. W. Kuhn. Extensive games and the problem of information. In H. W. Kuhn and A. W. Tucker, editors, Contributions to the Theory of Games, pages 196-216. Princeton University Press, Princeton, NJ, 1953.

[32] P. R. Kumar and P. Varaiya. Stochastic Systems. Prentice-Hall, Englewood Cliffs, NJ, 1986.

[33] H. Kurniawati, D. Hsu, and W. S. Lee. SARSOP: Efficient point-based POMDP planning by approximating optimally reachable belief spaces. In Proceedings Robotics: Science and Systems, 2008.

[34] A. Ladd, K. E. Bekris, A. Rudys, G. Marceau, L. E. Kavraki, and D. S. Wallach. Roboticsbased location sensing using wireless ethernet. In Proceedings of the 8th Annual International Conference on Mobile Computing and Networking, pages 227-238, Atlanta, 2002. 
[35] J.-C. Latombe. Robot Motion Planning. Kluwer, Boston, MA, 1991.

[36] J.-C. Latombe, A. Lazanas, and S. Shekhar. Robot motion planning with uncertainty in control and sensing. Artificial Intelligence Journal, 52:1-47, 1991.

[37] S. L. Laubach and J. W. Burdick. An autonomous sensor-based path-planning for planetary microrovers. In Proceedings IEEE International Conference on Robotics $\&$ Automation, 1999.

[38] S. M. LaValle. Planning Algorithms. Cambridge University Press, Cambridge, U.K., 2006. Also available at http://planning.cs.uiuc.edu/.

[39] T. Lozano-Pérez, M. T. Mason, and R. H. Taylor. Automatic synthesis of fine-motion strategies for robots. International Journal of Robotics Research, 3(1):3-24, 1984.

[40] V. J. Lumelsky and T. Skewis. A paradigm for incorporating vision in the robot navigation function. In Proceedings IEEE International Conference on Robotics \& Automation, pages 734-739, 1988.

[41] V. J. Lumelsky and A. A. Stepanov. Path planning strategies for a point mobile automaton moving amidst unknown obstacles of arbitrary shape. Algorithmica, 2:403-430, 1987.

[42] M. T. Mason. Mechanics of Robotic Manipulation. MIT Press, Cambridge, MA, 2001.

[43] L. Murphy and P. Newman. Using incomplete online metric maps for topological exploration with the gap navigation tree. In Proceedings IEEE International Conference on Robotics $\&$ Automation, 2008.

[44] J. M. O'Kane and S. M. LaValle. On comparing the power of robots. International Journal of Robotics Research, 27(1):5-23, 2008.

[45] R. Platt, R. Tedrake, T. Lozano-Perez, and L. Pack Kaelbling. Belief space planning assuming maximum likelihood observations. In Proceedings Robotics: Science and Systems, 2010.

[46] S. Sastry. Nonlinear Systems: Analysis, Stability, and Control. Springer-Verlag, Berlin, 1999.

[47] M. W. Spong, S. Hutchinson, and M. Vidyasagar. Robot Modeling and Control. Wiley, New York, 2005.

[48] I. Suzuki and M. Yamashita. Searching for a mobile intruder in a polygonal region. SIAM Journal on Computing, 21(5):863-888, October 1992.

[49] K. Taylor and S. M. LaValle. I-Bug: An intensity-based bug algorithm. In Proceedings IEEE International Conference on Robotics and Automation, 2009.

[50] S. Thrun, W. Burgard, and D. Fox. Probabilistic Robotics. MIT Press, Cambridge, MA, 2005.

[51] B. Tovar, F. Cohen, and S. M. LaValle. Sensor beams, obstacles, and possible paths. In Proceedings Workshop on Algorithmic Foundations of Robotics (WAFR), 2008.

[52] B. Tovar, R Murrieta-Cid, and S. M. LaValle. Distance-optimal navigation in an unknown environment without sensing distances. IEEE Transactions on Robotics, 23(3):506-518, June 2007.

[53] J. von Neumann and O. Morgenstern. Theory of Games and Economic Behavior. Princeton University Press, Princeton, NJ, 1944. 
[54] J. G. Webster, editor. The Measurement, Instrumentation and Sensors Handbook. CRC Press, New York, 1999.

[55] J. Yu and S. M. LaValle. Tracking hidden agents through shadow information spaces. In Proceedings IEEE International Conference on Robotics and Automation, 2008. 